\title{
Birth, growth and computation of pi to ten trillion digits
}

\author{
Ravi P Agarwal ${ }^{1 *}$, Hans Agarwal ${ }^{2}$ and Syamal K Sen ${ }^{3}$
}

${ }^{\text {"Correspondence: }}$

Agarwal@tamuk.edu

1 Department of Mathematics, Texas A\&M University-Kingsville, Kingsville,

TX, 78363, USA

Full list of author information is

available at the end of the article

\begin{abstract}
The universal real constant pi, the ratio of the circumference of any circle and its diameter, has no exact numerical representation in a finite number of digits in any number/radix system. It has conjured up tremendous interest in mathematicians and non-mathematicians alike, who spent countless hours over millennia to explore its beauty and varied applications in science and engineering. The article attempts to record the pi exploration over centuries including its successive computation to ever increasing number of digits and its remarkable usages, the list of which is not yet closed.
\end{abstract}

Keywords: circle; error-free; history of pi; Matlab; random sequence; stability of a computer; trillion digits

All circles have the same shape, and traditionally represent the infinite, immeasurable and even spiritual world. Some circles may be large and some small, but their 'circleness', their perfect roundness, is immediately evident. Mathematicians say that all circles are similar. Before dismissing this as an utterly trivial observation, we note by way of contrast that not all triangles have the same shape, nor all rectangles, nor all people. We can easily imagine tall narrow rectangles or tall narrow people, but a tall narrow circle is not a circle at all. Behind this unexciting observation, however, lies a profound fact of mathematics: that the ratio of circumference to diameter is the same for one circle as for another. Whether the circle is gigantic, with large circumference and large diameter, or minute, with tiny circumference and tiny diameter, the relative size of circumference to diameter will be exactly the same. In fact, the ratio of the circumference to the diameter of a circle produces, the most famous/studied/unlimited praised/intriguing/ubiquitous/external/mysterious mathematical number known to the human race. It is written as pi or as $\pi$ [1-210], symbolically, and defined as

$$
\mathrm{pi}=\frac{\text { distance around a circle }}{\text { distance across and through the center of the circle }}=\frac{C}{D}=\pi \text {. }
$$

Since the exact date of birth of $\pi$ is unknown, one could imagine that $\pi$ existed before the universe came into being and will exist after the universe is gone. Its appearance in the disks of the Moon and the Sun, makes it as one of the most ancient numbers known to humanity. It keeps on popping up inside as well as outside the scientific community, for example, in many formulas in geometry and trigonometry, physics, complex analysis, cosmology, number theory, general relativity, navigation, genetic engineering, statistics,

(c) 2013 Agarwal et al.: licensee Springer. This is an Open Access article distributed under the terms of the Creative Commons Attribution License (http://creativecommons.org/licenses/by/2.0), which permits unrestricted use, distribution, and reproduction in any medium, provided the original work is properly cited. 
fractals, thermodynamics, mechanics, and electromagnetism. Pi hides in the rainbow, and sits in the pupil of the eye, and when a raindrop falls into water $\pi$ emerges in the spreading rings. Pi can be found in waves and ripples and spectra of all kinds and, therefore, $\pi$ occurs in colors and music. The double helix of DNA revolves around $\pi$. Pi has lately turned up in super-strings, the hypothetical loops of energy vibrating inside subatomic particles. Pi has been used as a symbol for mathematical societies and mathematics in general, and built into calculators and programming languages. $\mathrm{Pi}$ is represented in the mosaic outside the mathematics building at the Technische Universität Berlin. Pi is also engraved on a mosaic at Delft University. Even a movie has been named after it. Pi is the secret code in Alfred Hitchcock's 'Torn Curtain' and in 'The Net' starring Sandra Bullock. Pi day is celebrated on March 14 (which was chosen because it resembles 3.14). The official celebration begins at 1:59 p.m., to make an appropriate 3.14159 when combined with the date. In 2009, the United States House of Representatives supported the designation of Pi Day. Albert Einstein was born on Pi Day (14 March 1879).

Throughout the history of $\pi$, which according to Beckmann (1971) 'is a quaint little mirror of the history of man', and James Glaisher (1848-1928) 'has engaged the attention of many mathematicians and calculators from the time of Archimedes to the present day, and has been computed from so many different formula, that a complete account of its calculation would almost amount to a history of mathematics', one of the enduring challenges for mathematicians has been to understand the nature of the number $\pi$ (rational/irrational/transcendental), and to find its exact/approximate value. The quest, in fact, started during the pre-historic era and continues to the present day of supercomputers. The constant search by many including the greatest mathematical thinkers that the world produced, continues for new formulas/bounds based on geometry/algebra/analysis, relationship among them, relationship with other numbers such as $\pi=5 \cos ^{-1}(\phi / 2), \pi \simeq$ $4 / \sqrt{\phi}$, where $\phi$ is the Golden section (ratio), and $e^{i \pi}+1=0$, which is due to Euler and contains 5 of the most important mathematical constants, and their merit in terms of computation of digits of $\pi$. Right from the beginning until modern times, attempts were made to exactly fix the value of $\pi$, but always failed, although hundreds constructed circle squares and claimed the success. These amateur mathematicians have been called the sufferers of morbus cyclometricus, the circle-squaring disease. Stories of these contributors are amusing and at times almost unbelievable. Many came close, some went to tens, hundreds, thousands, millions, billions, and now up to ten trillion $\left(10^{13}\right)$ decimal places, but there is no exact solution. The American philosopher and psychologist William James (1842-1910) wrote in 1909 'the thousandth decimal of Pi sleeps there though no one may ever try to compute it'. Thanks to the twentieth and twenty-first century, mathematicians and computer scientists, it sleeps no more. In 1889, Hermann Schubert (1848-1911), a Hamburg mathematics professor, said 'there is no practical or scientific value in knowing more than the 17 decimal places used in the foregoing, already somewhat artificial, application, and according to Arndt and Haenel (2000), just 39 decimal places would be enough to compute the circumference of a circle surrounding the known universe to within the radius of a hydrogen atom. Further, an expansion of $\pi$ to only 47 decimal places would be sufficiently precise to inscribe a circle around the visible universe that does not deviate from perfect circularity by more than the distance across a single proton. The question has been repeatedly asked why so many digits? Perhaps the primary motivation for these computations is the human desire to break records; the extensive calculations involved 
have been used to test supercomputers and high-precision multiplication algorithms (a stress test for a computer, a kind of 'digital cardiogram'), the statistical distribution of the digits, which is expected to be uniform, that is, the frequency with which the digits (0 to 9) appear in the result will tend to the same limit (1/10) as the number of decimal places increases beyond all bounds, and in recent years these digits are being used in applied problems as a random sequence. It appears experts in the field of $\pi$ are looking for surprises in the digits of $\pi$. In fact, the Chudnovsky brothers once said: 'We are looking for the appearance of some rules that will distinguish the digits of $\pi$ from other numbers. If you see a Russian sentence that extends for a whole page, with hardly a comma, it is definitely Tolstoy. If someone gave you a million digits from somewhere in $\pi$, could you tell it was from $\pi$ '? Some interesting observations are: The first 144 digits of $\pi$ add up to 666 (which many scholars say is 'the mark of the Beast'); Since there are 360 degrees in a circle, some mathematicians were delighted to discover that the number 360 is at the 359th digit position of $\pi$. A mysterious 2008 crop circle in Britain shows a coded image representing the first 10 digits of $\pi$. The Website 'The Pi-Search Page' finds a person's birthday and other well-known numbers in the digits of $\pi$. Several people have endeavored to memorize the value of $\pi$ with increasing precision, leading to records of over 100,000 digits.

We believe that the study and discoveries of $\pi$ will never end; there will be books, research articles, new record-setting calculations of the digits, clubs and computer programs dedicated to $\pi$. In what follows, we shall discuss the growth and the computation of $\pi$ chronologically. For our ready reference, we also give some digits of $\pi$,

$$
\pi=3.14159265358979323846264338327950288419716939937510
$$$$
58209749445923078164062862089986280348253421170679 .
$$

About 3200 BC. The meaning of the word sulv is to measure, and geometry in ancient India came to be known by the name sulba or sulva. Sulbasutras means 'rule of chords', which is another name for geometry. The Sulbasutras are part of the larger corpus of texts called the Shrautasutras, considered to be appendices to the Vedas, which give rules for constructing altars. If the ritual sacrifice was to be successful, then the altar had to conform to very precise measurements, so mathematical accuracy was seen to be of the utmost importance. The sulbas contain a large number of geometric constructions for squares, rectangles, parallelograms and trapezia. Sulbas also contain remarkable approximations

$$
\sqrt{2} \simeq 1+\frac{1}{3}+\frac{1}{3 \cdot 4}-\frac{1}{3 \cdot 4 \cdot 34}
$$

which gives $\sqrt{2}=1.4142156 \ldots$, and

$$
\pi \simeq 18(3-2 \sqrt{2})=\left(\frac{6}{2+\sqrt{2}}\right)^{2}
$$

which gives $\pi=3.088311 \ldots$.

About 2742 BC. Aryabhatta was born in 2765 BC in Patliputra in Magadha, modern Patna in Bihar (India). He was teaching astronomy and mathematics when he was 23 years of age in $2742 \mathrm{BC}$. His astronomical knowledge was so advanced that he could claim that the Earth rotated on its own axis, the Earth moves round the Sun and the Moon rotates 
round the Earth; incredibly he believed that the orbits of the planets are ellipses. He talks about the position of the planets in relation to its movement around the Sun. He refers to the light of the planets and the Moon as reflection from the Sun. He explains the eclipse of the Moon and the Sun, day and night, the length of the year exactly as 365 days. He calculated the circumference of the Earth as 24,835 miles, which is close to modern day calculation of 24,900 miles. In his Aryabhattiyam, which consists of the 108 verses and 13 introductory verses, and is divided into four padas or chapters (written in the very terse style typical of sutra literature, in which each line is an aid to memory for a complex system), Aryabhatta included 33 verses giving 66 mathematical rules ganita on pure mathematics. He described various original ways to perform different mathematical operations, including square and cube roots and solving quadratic equations. He provided elegant results for the summation of series of squares and cubes. He made use of decimals, the zero (sunya) and the place value system. To find an approximate value of $\pi$, Aryabhatta gives the following prescription: Add 4 to 100, multiply by 8 and add to 62,000. This is 'approximately' the circumference of a circle whose diameter is 20,000. This means $\pi=62,832 / 20,000=3.1416$. It is important to note that Aryabhatta used the word asanna (approaching), to mean that not only is this an approximation of $\pi$, but that the value is incommensurable or irrational, i.e., it cannot be expressed as a ratio of two integers.

About 2600 BC. Great pyramid at Gizeh was built around 2600 BC in Egypt. It is one of the most massive buildings ever erected. It has at least twice the volume and thirty times the mass (the resistance an object offers to a change in its speed or direction of motion) of the Empire Sate Building in New York, and built from individual stones weighing up to 70 tons each. From the dimensions of the Great Pyramid, it is possible to derive the value of $\pi$, namely, $\pi=$ half the perimeter of the base of the pyramid, divided by its height $=3+$ $1 / 7 \simeq 3.14285 \ldots$.

About 2000 BC. In a tablet found in 1936 in Susa (Iraq), Babylonians used the value

$$
\frac{3}{\pi}=\frac{57}{60}+\frac{36}{(60)^{2}}
$$

which yields $\pi=31 / 8=3.125$. They were also satisfied with $\pi=3$.

About 2000 BC. Ahmes (around 1680-1620 BC) (more accurately Ahmose) was an Egyptian scribe. A surviving work of Ahmes is part of the Rhind Mathematical Papyrus, 1650 BC (named after the Scottish Egyptologist Alexander Henry Rhind who went to Thebes for health reasons, became interested in excavating and purchased the papyrus in Egypt in 1858) located in the British Museum since 1863. When new, this papyrus was about 18 feet long and 13 inches high. Ahmes states that he copied the papyrus from a nowlost Middle Kingdom original, dating around 2000 BC. This curious document entitled directions for knowing all dark things, deciphered by Eisenlohr in 1877, is a collection of problems in geometry and arithmetic, algebra, weights and measures, business and recreational diversions. The 87 problems are presented with solutions, but often with no hint as to how the solution was obtained. In problem no. 50 , Ahmes states that a circular field with a diameter of 9 units in area is the same as a square with sides of 8 units, i.e., $\pi(9 / 2)^{2}=8^{2}$, and hence the Egyptian value of $\pi$ is

$$
\pi=4 \times\left(\frac{8}{9}\right)^{2}=3.16049 \ldots,
$$


which is only very slightly worse than the Babylonians value, and in contrast to the latter, an overestimation. We have no idea how this very satisfactory result was obtained (probably empirically), although various justifications are available. Maya value of $\pi$ was as good as that of the Egyptians.

About 1200 BC. The earliest Chinese mathematicians, from the time of Chou-Kong used the approximation $\pi=3$. Some of those who used this approximation were mathematicians of considerable attainments in other respects. According to the Chinese mythology, 3 is used because it is the number of the Heavens and the circle.

About 950 BC. In the Old Testament (I Kings vii.23, and 2 Chronicles iv.2), we find the following verse: 'Also, he made a molten sea of ten cubits from brim to brim, round in compass, and five cubits the height thereof; and a line of thirty cubits did compass it round about.' Hence the biblical value of $\pi$ is $30 / 10=3$. The Jewish Talmud, which is essentially a commentary on the Old Testament, was published about 500 AD. This shows that the Jews did not pay much attention to geometry. However, debates have raged on for centuries about this verse. According to some, it was just a simple approximation, while others say that ' ...the diameter perhaps was measured from outside, while the circumference was measured from inside'.

About 900 BC. Shatapatha Brahmana (Priest manual of 100 paths) is one of the prose texts describing the Vedic ritual. It survives in two recensions, Madhyandina and Kanva, with the former having the eponymous 100 brahmanas in 14 books, and the latter 104 brahmanas in 17 books. In these books, $\pi$ is approximated by $339 / 108=3.138888 \ldots$.

About 440 BC. Anaxagoras of Clazomanae (500-428 BC) came to Athens from near Smyrna, where he taught the results of the Ionian philosophy. He neglected his possessions in order to devote himself to science, and in reply to the question, what was the object of being born, he remarked: 'The investigation of the Sun, Moon and heaven'. He was the first to explain that the Moon shines due to reflected light from the Sun, which explains the Moon's phases. He also said that the Moon had mountains and he believed that it was inhabited. Anaxagoras gave some scientific accounts of eclipses, meteors, rainbows, and the Sun, which he asserted was larger than the Peloponnesus: this opinion, and various other physical phenomena, which he tried to explain which were supposed to have been direct action of the Gods, led him to a prosecution for impiety. While in prison he wrote a treatise on the quadrature of the circle. (The general problem of squaring a figure came to be known as the quadrature problem.) Since that time, hundreds of mathematicians tried to find a way to draw a square with equal area to a given circle; some maintained that they have found methods to solve the problem, while others argued that it is impossible. We will see that the problem was finally laid to rest in the nineteenth century.

About 430 BC. Hippocrates of Chios was born about 470 BC, and began life as a merchant. About $430 \mathrm{BC}$ he came to Athens from Chios and opened a school of geometry, and began teaching, thus became one of the few individuals ever to enter the teaching profession for its financial rewards. He established the formula $\pi r^{2}$ for the area of a circle in terms of its radius. It means that a certain number $\pi$ exists, and is the same for all circles, although his method does not give the actual numerical value of $\pi$. In trying to square the circle (unsuccessfully), Hippocrates discovered that two moon-shaped figures (lunes, bounded by pair of circular arcs) could be drawn whose areas were together equal to that of a right-angled triangle. Hippocrates gave the first example of constructing a rectilinear area equal to an area bounded by one or more curves. 
About 430 BC. Antiphon of Rhamnos (around 480-411 BC) was a sophist who attempted to find the area of a circle by considering it as the limit of an inscribed regular polygon with an infinite number of sides. Thus, he provided preliminary concept of infinitesimal calculus.

About 420 BC. Bryson of Heraclea was born around 450 BC. He was a student of Socrates. Bryson considered the circle squaring problem by comparing the circle to polygons inscribed within it. He wrongly assumed that the area of a circle was the arithmetical mean between circumscribed and inscribed polygons.

About 420 BC. Hippias of Elis was born about 460 BC. He was a Greek Sophist, a younger contemporary of Socrates. He is described as an expert arithmetician, but he is best known to us through his invention of a curve called the quadratrix $(x=y \cot (\pi y / 2))$, by means of which an angle can be trisected, or indeed divided in any given ratio. It is not known whether Hippias realized that by means of his curve the circle could be squared; perhaps he realized but could not prove it. He lectured widely on mathematics and as well on poetry, grammar, history, politics, archeology and astronomy. Hippias was also a prolific writer, producing elegies, tragedies and technical treatises in prose. His work on Homer was considered excellent.

414 BC. Aristophanes (446-386 BC) in his play The Birds makes fun of circle squarers. Around 375 BC. Plato of Athens (around 427-347 BC) was one of the greatest Greek philosophers, mathematicians, mechanician, a pupil of Socrates for eight years, and teacher of Aristotle. He is famous for 'Plato's Academy'. 'Let no man ignorant of mathematics enter here' is supposed to have been inscribed over the doors of the Academy. He is supposedly obtained for his day a fairly accurate value for $\pi=\sqrt{2}+\sqrt{3}=3.146 \ldots$.

About 370 BC. Eudoxus of Cnidus (around 400-347 BC) was the most celebrated mathematician. He developed the theory of proportion, partly to place the doctrine of incommensurables (irrationals) upon a thoroughly sound basis. Specially, he showed that the area of a circle is proportional to its diameter squared. Eudoxus established fully the method of exhaustions of Antiphon by considering both the inscribed and circumscribed polygons. He also considered certain curves other than the circle. He explained the apparent motions of the planets as seen from the earth. Eudoxus also wrote a treatise on practical astronomy, in which he supposed a number of moving spheres to which the Sun, Moon and stars were attached, and which by their rotation produced the effects observed. In all, he required 27 spheres.

About 350 BC. Dinostratus (around 390-320 BC) was a Greek mathematician. He used Hippias quadratrix to square the circle. For this, he proved Dinostratus' theorem. Hippias quadratrix later became known as the Dinostratus quadratrix also. However, his demonstration was not accepted by the Greeks as it violated the foundational principles of their mathematics, namely, using only ruler and compass.

About 240 BC. Archimedes of Syracuse (287-212 BC) ranks with Newton and Gauss as one of the three greatest mathematicians who ever lived, and he is certainly the greatest mathematician of antiquity. Galileo called him 'divine Archimedes, superhuman Archimedes'; Sir William Rowan Hamilton (1805-1865) remarked 'who would not rather have the fame of Archimedes than that of his conqueror Marcellus'?; Alfred North Whitehead (1861-1947) commented 'no Roman ever died in contemplation over a geometrical diagram'; Godfrey Harold Hardy (1877-1947) said 'Archimedes will be remembered when Aeschylus is forgotten, because languages die and mathematical ideas do not'; and 


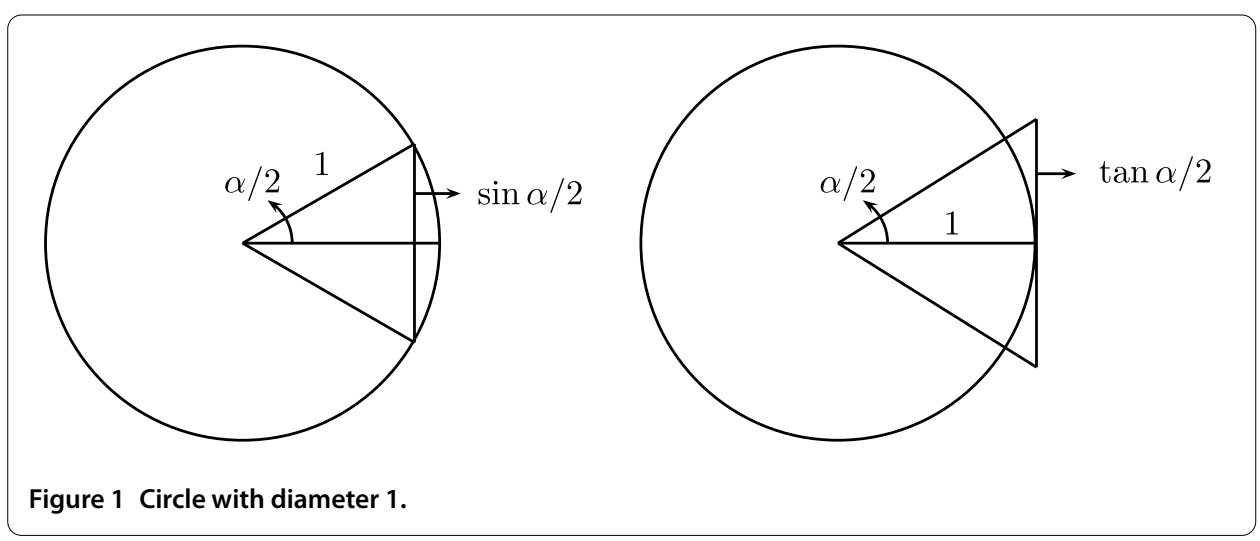

Voltaire remarked 'there was more imagination in the head of Archimedes than in that of Homer.' His mathematical work is so modern in spirit and technique that it is barely distinguishable from that of a seventeenth-century mathematician. Among his mathematical achievements, Archimedes developed a general method of exhaustion for finding areas bounded by parabolas and spirals, and volumes of cylinders, parabolas, segments of spheres, and specially to approximate $\pi$, which he called as the parameter to diameter. His approach to approximate $\pi$ is based on the following fact: the circumference of a circle lies between the perimeters of the inscribed and circumscribed regular polygons (equilateral and equiangular) of $n$ sides, and as $n$ increases, the deviation of the circumference from the two perimeters becomes smaller. Because of this fact, many mathematicians claim that it is more correct to say that a circle has an infinite number of corners than to view a circle as being cornerless. If $a_{n}$ and $b_{n}$ denote the perimeters of the inscribed and circumscribed regular polygons of $n$ sides, and $C$ the circumference of the circle, then it is clear that $\left\{a_{n}\right\}$ is an increasing sequence bounded above by $C$, and $\left\{b_{n}\right\}$ is a decreasing sequence bounded below by $C$. Both of these sequences converge to the same limit $C$. To simplify matters, suppose we choose a circle with the diameter 1, then from Figure 1 it immediately follows that

$$
a_{n}=n \sin \frac{\pi}{n} \quad \text { and } \quad b_{n}=n \tan \frac{\pi}{n} .
$$

It is clear that $\lim _{n \rightarrow \infty} a_{n}=\pi=\lim _{n \rightarrow \infty} b_{n}$. Further, $b_{2 n}$ is the harmonic mean of $a_{n}$ and $b_{n}$, and $a_{2 n}$ is the geometric mean of $a_{n}$ and $b_{2 n}$, i.e.,

$$
b_{2 n}=\frac{2 a_{n} b_{n}}{a_{n}+b_{n}} \quad \text { and } \quad a_{2 n}=\sqrt{a_{n} b_{2 n}} \text {. }
$$

From (1) for the hexagon, i.e., $n=6$ it follows that $a_{6}=3, b_{6}=2 \sqrt{3}$. Then Archimedes successively took polygons of sides $12,24,48$ and 96 , used the recursive relations (2), and the inequality

$$
\frac{265}{153}<\sqrt{3}<\frac{1,351}{780},
$$

which he probably found by what is now called Heron's method, to obtain the bounds

$$
3.140845 \ldots=3 \frac{10}{71}<\pi<3 \frac{1}{7}=3.142857 \ldots
$$


It is interesting to note that during Archimedes time algebraic and trigonometric notations, and our present decimal system were not available, and hence he had to derive recurrence relations (2) geometrically, and certainly for him the computation of $a_{96}$ and $b_{96}$ must have been a formidable task. The approximation $22 / 7$ is often called the Archimedean value of $\pi$, and it is good for most purposes. If we take the average of the bounds given in (3), we obtain $\pi=3.141851 \ldots$. The above method of computing $\pi$ by using regular inscribed and circumscribed polygons is known as the classical method of computing $\pi$. It follows that an inscribed regular polygon of $2^{n}$ sides takes up more than $1-1 / 2^{n-1}$ of the area of a circle. Heron of Alexandria (about 75 AD) in his Metrica, which had been lost for centuries until a fragment was discovered in 1894 , followed by a complete copy in 1896, refers to an Archimedes work, where he gives the bounds

$$
3.14163 \ldots=\frac{211,875}{67,441}<\pi<\frac{197,888}{62,351}=3.173774 \ldots .
$$

Clearly, in the above right inequality, there is a mistake as it is worse than the upper bound 22/7 found by Archimedes earlier. Heron adds 'Since these numbers are inconvenient for measurements, they are reduced to the ratio of the smaller numbers, namely, 22/7. Archimedes' polygonal method remained unsurpassed for 18 centuries. Archimedes also showed that a curve discovered by Conon of Samos (around 280-220 BC) could, like Hippias' quadratrix, be used to square the circle. The curve is today called the Archimedean Spiral.

About 123 BC. Daivajna Varahamihira (working 123 BC) was an astronomer, mathematician and astrologer. His picture may be found in the Indian Parliament along with Aryabhata. He was one of the nine jewels (Navaratnas) of the court of legendary king Vikramaditya I (101-18 BC). In 123 BC, Varahamihira wrote Pancha-Siddhanta (The Five Astronomical Canons), in which he codified the five existing Siddhantas, namely, Paulisa Siddhanta, Romaka Siddhanta, Vasishtha Siddhanta, Surya Siddhanta and Paitamaha Siddhanta. He also made some important mathematical discoveries such as giving certain trigonometric formulae; developing new interpolation methods to produce sine tables; constructing a table for the binomial coefficients; and examining the pandiagonal magic square of order four. In his work, he approximated $\pi$ as $\sqrt{10}$.

15 BC. Marcus Vitruvius Pollio (about 80-5 BC), a Roman writer, architect and engineer, in his multi-volume work De Architectura (On Architecture) used the value $\pi=31 / 8=$ 3.125, which is the same as Babylonians had used 2,000 years earlier. He was the first to describe direct measurement of distances by the revolution of a wheel.

About 10 BC. Liu Xin (Liu Hsin) (about 50 BC-23 AD) was an astronomer, historian and editor during the Xin Dynasty (9-23 AD). Liu created a new astronomical system, called Triple Concordance. He was the first to give a more accurate calculation of $\pi$ as 3.1547, the exact method he used to reach this figure is unknown. This was first mentioned in the Sui shu (387-388). He also found the approximations 3.1590, 3.1497 and 3.1679.

Around 5 AD. Liu Xin (50 BC-AD 23) was a Chinese astronomer, historian and editor during the Xin Dynasty (9-23 AD). He was the son of Confucian scholar Liu Xiang (77-6 $\mathrm{BC})$. Liu created a catalog of 1,080 stars, where he used the scale of 6 magnitudes. He was the first in China to give a more accurate calculation of $\pi$ as 3.1457. The method he used to reach this figure is unknown. 
10 AD. Brahmagupta (born $30 \mathrm{BC}$ ) wrote two treatises on mathematics and astronomy: the Brahmasphutasiddhanta (The Correctly Established Doctrine of Brahma) but often translated as (The Opening of the Universe), and the Khandakhadyaka (Edible Bite) which mostly expands the work of Aryabhata. As a mathematician he is considered as the father of arithmetic, algebra, and numerical analysis. Most importantly, in Brahmasphutasiddhanta he treated zero as a number in its own right, stated rules for arithmetic on negative numbers and zero, and attempted to define division by zero, particularly he wrongly believed that $0 / 0$ was equal to 0 . He used a geometric construction for squaring the circle, which amounts to $\pi=\sqrt{10}$.

125. Zhang Heng (78-139 AD) was an astronomer, mathematician, inventor, geographer, cartographer, artist, poet, statesman and literary scholar. He proposed a theory of the universe that compared it to an egg. 'The sky is like a hen's egg and is as round as a crossbow pellet. The Earth is like the yolk of the egg, lying alone at the center. The sky is large and the Earth is small'. According to him the universe originated from chaos. He said that the Sun, Moon and planets were on the inside of the sphere and moved at different rates. He demonstrated that the Moon did not have independent light, but that it merely reflected the light from the sun. He is most famous in the West for his rotating celestial globe, and inventing in 132 the first seismograph for measuring earthquakes. He proposed $\sqrt{10}$ (about 3.1623) for $\pi$. He also compared the celestial circle to the width (i.e., diameter) of the earth in the proportion of 736 to 232 , which gives $\pi$ as 3.1724 .

150. Claudius Ptolemaeus (around 90-168 AD) known in English as Ptolemy, was a mathematician, geographer, astrologer, poet of a single epigram in the Greek Anthology, and most importantly astronomer. He made a map of the ancient world in which he employed a coordinate system very similar to the latitude and longitude of today. One of his most important achievements was his geometric calculations of semichords. Ptolemy in his famous Syntaxis mathematica (more popularly known by its Arabian title of the Almagest), the greatest ancient Greek work on astronomy, obtained, using chords of a circle and an inscribed 360-gon, an approximate value of $\pi$ in sexagesimal notation, as $38^{\prime} 30^{\prime \prime}$, which is the same as $377 / 120=3.141666 \ldots$ Eutocius of Ascalon (about 480-540) refers to a book Quick delivery by Apollonius of Perga (around 262-200 BC), who earned the title 'The Great Geometer', in which Apollonius obtained an approximation for $\pi$, which was better than known to Archimedes, perhaps the same as 377/120.

250. Wang Fan (228-266) was a mathematician and astronomer. He calculated the distance from the Sun to the Earth, but his geometric model was not correct. He has been credited with the rational approximation $142 / 45$ for $\pi$, yielding $\pi=3.155$.

263. Liu Hui (around 220-280) wrote two works. The first one was an extremely important commentary on the Jiuzhang suanshu, more commonly called Nine Chapters on the Mathematical Art, which came into being in the Eastern Han Dynasty, and believed to have been originally written around $1000 \mathrm{BC}$. (It should be noted that very little is known about the mathematics of ancient China. In 213 BC, the emperor Shi Huang of the Chin dynasty had all of the manuscript of the kingdom burned.) The other was a much shorter work called Haidao suanjing or Sea Island Mathematical Manual. In Jiuzhang suanshu, Liu Hui used a variation of the Archimedean inscribed regular polygon with 192 sides to approximate $\pi$ as 3.141014 and suggested $157 / 50=3.14$ as a practical approximation.

About 330. Pappus of Alexandria (around 290-350) was born in Alexandria, Egypt, and either he was a Greek or a Hellenized Egyptian. The written records suggest that, Pappus 
lived in Alexandria during the reign of Diocletian (284-305). His major work is Synagoge or the Mathematical Collection, which is a compendium of mathematics of which eight volumes have survived. Pappus' Book IV contains various theorems on circles, study of various curves, and an account of the three classical problems of antiquity (the squaring of the circle, the duplication of a cube, and the trisection of an angle). For squaring the circle, he used Dinostratus quadratrix and his proof is a reductio ad absurdum. Pappus is remembered for Pappus's centroid theorem, Pappus's chain, Pappus's harmonic theorem, Pappus's hexagon theorem, Pappus's trisection method, and for the focus and directrix of an ellipse.

400. He Chengtian (370-447) gave the approximate value of $\pi$ as $111,035 / 35,329=$ $3.142885 \ldots$.

475. Tsu Ch'ung-chih (Zu Chongzhi) (429-500) created various formulas that have been used throughout history. With his son he used a variation of Archimedes method to find $3.1415926<\pi<3.1415927$. He also obtained a remarkable rational approximation $355 / 113$, which yields $\pi$ correct to six decimal digits. In Chinese this fraction is known as Milü. To compute this accuracy for $\pi$, he must have taken an inscribed regular $6 \times 2^{12}$ gon and performed lengthy calculations. Note that $\pi=355 / 113$ can be obtained from the values of Ptolemy and Archimedes:

$$
\frac{355}{113}=\frac{377-22}{120-7}
$$

He declared that 22/7 is an inaccurate value whereas $355 / 113$ is the accurate value of $\pi$. We also note that $\pi=355 / 113$ can be obtained from the values of Liu Hui and Archimedes. In fact, by using the method of averaging, we have

$$
\frac{157+(9 \times 22)}{50+(9 \times 7)}=\frac{355}{113}
$$

486. Bhaskara II or Bhaskaracharya (working 486) wrote Siddhanta Siromani (crown of treatises), which consists of four parts, namely, Leelavati Bijaganitam, Grahaganitam and Goladhyaya. The first two exclusively deal with mathematics and the last two with astronomy. His popular text Leelavati was written in $486 \mathrm{AD}$ in the name of his daughter. His contributions to mathematics include: a proof of the Pythagorean theorem, solutions of quadratic, cubic, and quartic indeterminate equations, solutions of indeterminate quadratic equations, integer solutions of linear and quadratic indeterminate equations, a cyclic Chakravala method for solving indeterminate equations, solutions of the Pell's equation and solutions of Diophantine equations of the second order. He solved quadratic equations with more than one unknown, and found negative and irrational solutions, provided preliminary concept of infinitesimal calculus, along with notable contributions toward integral calculus, conceived differential calculus, after discovering the derivative and differential coefficient, stated Rolle's theorem, calculated the derivatives of trigonometric functions and formulae and developed spherical trigonometry. He conceived the modern mathematical convention that when a finite number is divided by zero, the result is infinity. He speculated the nature of the number $1 / 0$ by stating that it is 'like the Infinite, Invariable God who suffers no change when old worlds are destroyed or new ones created, when innumerable species of creatures are born or as many perish'. He gave several 
approximations for $\pi$. According to him 3,927/1,250 is an accurate value, 22/7 is an inaccurate value, and $\sqrt{10}$ is for ordinary work. The first value may have been taken from Aryabhatta. This approximation has also been credited to Liu Hui and $\mathrm{Zu}$ Chongzhi. He also gave the value $754 / 240=3.1416$, which is of uncertain origin; however, it is the same as that by Ptolemy.

510. Anicius Manlius Severinus Boethius (around 475-526) introduced the public use of sun-dials, water-clocks, etc. His integrity and attempts to protect the provincials from the plunder of the public officials brought on him the hatred of the Court. King Theodoric sentenced him to death while absent from Rome, seized at Ticinum (now Pavia), and in the baptistery of the church there tortured by drawing a cord round his head till the eyes were forced out of the sockets, and finally beaten to death with clubs on October 23, 526 . His Geometry consists of the enunciations (only) of the first book of Euclid, and of a few selected propositions in the third and fourth books, but with numerous practical applications to finding areas, etc. According to him, the circle had been squared in the period since Aristotle's time, but noted that the proof was too long.

800. Abu Jafar Mohammed Ibn Musa al-Khwarizmi (around 780-850) 'Mohammed the father of Jafar and the son of Musa' was a scholar in the academy Bait al-Hikma (House of Wisdom) founded by Caliph al-Mamun (786-833). His task (along with several other scholars) was to translate the Greek and Sanskrit scientific manuscripts. They also studied, and wrote on algebra, geometry and astronomy. There al-Khwarizmi encountered the Hindu place-value system based on the numerals $0,1,2,3,4,5,6,7,8,9$, including the first use of zero as a place holder in positional base notation, and he wrote a treatise around $820 \mathrm{AD}$, on what we call Hindu-Arabic numerals. The Arabic text is lost but a Latin translation, Algoritmi de numero Indorum (that is, al-Khwarizmi on the Hindu Art of Reckoning), a name given to the work by Baldassarre Boncompagni in 1857, much changed from al-Khwarizmi's original text (of which even the title is unknown) is known. The French Minorite friar Alexander de Villa Dei, who taught in Paris around 1240, mentions the name of an Indian king named Algor as the inventor of the new 'art', which itself is called the algorismus. Thus, the word 'algorithm' was tortuously derived from al-Khwarizmi (Alchwarizmi, al-Karismi, Algoritmi, Algorismi, Algorithm), and has remained in use to this day in the sense of an arithmetic operation. This Latin translation was crucial in the introduction of Hindu-Arabic numerals to medieval Europe. Al-Khwarizmi used $\pi=22 / 7$ in algebra, $\pi=\sqrt{10}$ in geometry, and $\pi=62,832 / 20,000=3.1416$ in astronomy.

850. Mahavira (817-875) in his work Ganita Sara Samgraha summarized and extended the works of Aryabhatta, Bhaskara, Brahmagupta and Bhaskaracharya. This treatise contains: a naming scheme for numbers from 10 up to $10^{24}$, formulas for obtaining cubes of sums; techniques for least common denominators (LCM), techniques for combinations ${ }^{n} C_{r}=n(n-1)(n-2) \cdots(n-r+1) / r$ ! , techniques for solving linear, quadratic as well higher order equations, arithmetic and geometric series, and techniques for calculating areas and volumes. He was the first person to mention that no real square roots of negative numbers can exist. According to Mahavira whatever is there in all the three worlds, which are possessed of moving and non-moving beings, all that indeed cannot exist without mathematics. He used the approximate value of $\pi$ as $\sqrt{10}$. He also mentions that the approximate volume of a sphere with diameter $d$ is $(9 / 2)(d / 2)^{3}$, i.e., $\pi=3.375$, and exact volume is $(9 / 10)(9 / 2)(d / 2)^{3}$, i.e., $\pi=3.0375$. 
About 1040. Franco von Lüttich (around 1015-1083) claimed to have contributed the only important work in the Christian era on squaring the circle. His works are published in six books, but only preserved in fragments.

1220. Fibonacci (Leonardo of Pisa) (around 1170-1250) after the Dark Ages is considered the first to revive mathematics in Europe. He wrote Liber Abbaci (Book of the Abacus) in 1202. In this book, he quotes that 'The nine Indian numerals are... with these nine and with the sign 0 which in Arabic is sifr, any desired number can be written. His Practica geometria, a collection of useful theorems from geometry and (what would eventually be named) trigonometry appeared in 1220, which was followed five years later by Liber quadratorum, a work on indeterminate analysis. A problem in Liber Abbaci led to the introduction of the Fibonacci sequence for which he is best remembered today; however, this sequence earlier appeared in the works of Pingala (about $500 \mathrm{BC}$ ) and Virahanka (about 600 AD). In Practica geometriae, Fibonacci used a 96-sided polygon, to obtain the approximate value of $\pi$ as $864 / 275=3.141818 \ldots$.

1260. Johannes Campanus (around 1220-1296) was chaplain to three popes, Pope Urban IV, Pope Nicholas IV and Pope Boniface VIII. He was one of the four greatest contemporary mathematicians. Campanus wrote a Latin edition of Euclid's Elements in 15 books around 1260 . He used the value of $\pi$ as 22/7.

About 1300. Zhao Youqin (born 1271) used a regular polygon of $4 \times 2^{12}$ sides to derive $\pi=3.1415926$.

About 1360. Albert of Saxony (around 1320-1390) was a German philosopher known for his contributions to logic and physics. He wrote a long treatise De quadratura circuli (Question on the Squaring of the Circle) consisting mostly philosophy. He said 'following the statement of many philosophers, the ratio of circumference to diameter is exactly 22/7; of this, there is proof, but a very difficult one'.

1400. Madhava of Sangamagramma's (1340-1425) work has come to light only very recently. Although there is some evidence of mathematical activities in Kerala (India) prior to Madhava, e.g., the text Sadratnamala (about 1300), he is considered the founder of the Kerala school of astronomy and mathematics. Madhava was the first to have invented the ideas underlying infinite series expansions of functions, power series, trigonometric series of sine, cosine, tangent and arctangent, which is

$$
\tan ^{-1} x=x-\frac{x^{3}}{3}+\frac{x^{5}}{5}-\frac{x^{7}}{7}+\cdots+(-1)^{n-1} \frac{x^{2 n-1}}{2 n-1}+\cdots
$$

This series is valid for $-1<x<1$, and also for $x=1$. He also gave rational approximations of infinite series, tests of convergence of infinite series, estimate of an error term, early forms of differentiation and integration and the analysis of infinite continued fractions. He fully understood the limit nature of the infinite series. Madhava discovered the solutions of transcendental (transcends the power of algebra) equations by iteration, and found the approximation of transcendental numbers by continued fractions. He also gave many methods for calculating the circumference of a circle. The value of $\pi$ correct to 13 decimal places is attributed to Madhava. However, the text Sadratnamala, usually considered as prior to Madhava, while some researchers have claimed that it was compiled by Madhava, gives the astonishingly accurate value of $\pi$ correct to 17 decimal places.

1429. Jemshid al-Kashi (around 1380-1429), astronomer royal to Ulugh Beg of Samarkand, wrote several important books Sullam al-sama (The Stairway of Heaven), 
Mukhtasar dar 'ilm-i hay'at (Compendium of the Science of Astronomy), Khaqani Zij on astronomical tables, Risala dar sharh-i alat-i rasd (Treatise on the Explanation of Observational Instruments), Nuzha al-hadaiq fi kayfiyya san'a al-ala almusamma bi tabaq almanatiq (The Method of Construction of the Instrument Called Plate of Heavens), Risala al-muhitiyya (Treatise on the Circumference), The Key to Arithmetic, and The Treatise on the Chord and Sine. In these works al-Kashi showed a great venality in numerical work. In 1424 , he calculated $\pi$ to 14 decimal places, and later in 1429 to 16 decimal places. For this, he used classical polygon method of $6 \times 2^{27}$ sides.

1460. George Pürbach (1423-1461) whose real surname is unknown, was born in Pürbach, a town upon the confines of Bavaria and Austria. He studied under Nicholas de Cusa, and one of his most famous pupils is Regiomontanus. Pürbach wrote a work on planetary motions which was published in 1460; an arithmetic, published in 1511; and a table of eclipses, published in 1514. He calculated tables of sines for every minute of arc for a radius of 600,000 units. This table was published in 1541 . He approximated $\pi$ by the rational 62,832/20,000, which is exactly the same as given by Aryabhatta.

1464. Nicholas of Cusa (1401-1464) is often referred to as Nicolaus Cusanus and Nicholas of Kues (Cusa was a Latin place-name for a city on the Mosel). He was a German cardinal of the Roman Catholic Church, a philosopher, jurist, mathematician and an astronomer. Most of his mathematical ideas can be found in his essays, De Docta Ignorantia (Of Learned Ignorance), De Visione Dei (Vision of God) and On Conjectures. He made important contributions to the field of mathematics by developing the concepts of the infinitesimal and of relative motion. He gave the approximations of $\pi$ as $(3 / 4)(\sqrt{3}+\sqrt{6})$ and $24 \sqrt{21} / 35=3.142337 \ldots$ Nicholas thought this to be the exact value. Nicholas said, if we can approach the Divine only through symbols, then it is most suitable that we use mathematical symbols, for these have an indestructible certainty. He also said that no perfect circle can exist in the universe. In accordance with his wishes, his heart is within the chapel altar at the Cusanusstift in Kues.

1464. Johann Regiomontanus (Johannes Müller) (1436-1476) is considered as one of the most prominent mathematicians of his generation. He was the first to study Greek mathematical works in order to make himself acquainted with the methods of reasoning and results used there. He also well read the works of the Arab mathematicians. In most of this study, he compiled in his De Triangulis, which was completed in 1464, however, was published only in 1533. Regiomontanus used algebra to find solutions of geometrical problems. He criticized Nicholas of Cusa's approximations and methods to approximate the value of $\pi$ and gave the approximation 3.14343 .

About 1500. Nilakanthan Somayaji's (around 1444-1544) most notable work Tantrasangraha elaborates and extends the contributions of Madhava. He was also the author of Aryabhatiya-Bhashya, a commentary of the Aryabhatiya. Of great significance in Nilakanthan's work includes the inductive mathematical proofs, a derivation and proof of the arctangent trigonometric function, improvements and proofs of other infinite series expansions by Madhava, and in Sanskrit poetry the series

$$
\frac{\pi}{4}=1-\frac{1}{3}+\frac{1}{5}-\frac{1}{7}+\frac{1}{9}-\frac{1}{11}+\cdots,
$$

which follows from Madhava's series (4) when $x=1$. In the literature (5) is known as Gregory-Leibniz series. He also gave sophisticated explanations of the irrationality of 
$\pi$, the correct formulation for the equation of the center of the planets, and a heliocentric model of the solar system. If $s_{n}$ denotes the $n$th partial sum of (5), then $s_{1}=1$, $s_{10}=0.76045 \ldots, s_{100}=0.78289 \ldots, s_{1,000}=0.78514 \ldots, s_{10,000}=0.78537 \ldots$ and Roy North

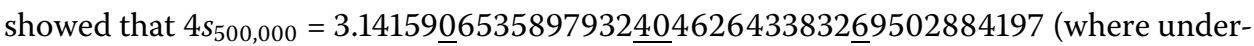
lined digits are incorrect) indicating an annoyingly slow convergence of the partial sums. Since this is an alternating series, the error committed by stopping at the $n$th term does not exceed $1 /(2 n+1)$ in absolute value. Thus, to compute $\pi / 4$ to eight decimals from (5) would require $n>10^{8}$ terms. Hence, although it is only of theoretical interest, the expressions on the right are arithmetical, while $\pi$ arises from geometry. We also note that the series (5) can be written as

$$
\frac{\pi}{4}=1-2\left(\frac{1}{3 \cdot 5}+\frac{1}{7 \cdot 9}+\frac{1}{11 \cdot 13}+\cdots\right)
$$

The following expansion of $\pi$ is also due to Nilakanthan

$$
\pi=3+\frac{4}{2 \cdot 3 \cdot 4}-\frac{4}{4 \cdot 5 \cdot 6}+\frac{4}{6 \cdot 7 \cdot 8}-\frac{4}{8 \cdot 9 \cdot 10}+\cdots
$$

This series converges faster than (5).

Before 1510. Leonardo da Vinci (1452-1519) was an Italian painter, sculptor, architect, musician, scientist, mathematician, engineer, inventor, anatomist, geologist, cartographer, botanist and writer. He briefly worked on squaring the circle, or approximating $\pi$.

1525. Michael Stifel (1486-1567) served in several different Churches at different positions; however, every time due to bad circumstances had to resign and flee. He made the error of predicting the end of the world on 3 October 1533, and other time used a clever rearrangement of the letters LEO DECIMVS to 'prove' that Leo X was 666, the number of the beast given in the Book of Revelation. He was forced to take refuge in a prison after ruining the lives of many believing peasants who had abandoned work and property to accompany him to heaven. In the later part of his life, he lectured on mathematics and theology. He invented logarithms independently of Napier using a totally different approach. His most famous work is Arithmetica integra which was published in 1544. This work contains binomial coefficients, multiplication by juxtaposition, the term 'exponent', and the notation,+- and $\sqrt{ }$, and the opinion that the quadrature of $\pi$ is impossible. According to him 'the quadrature of the circle is obtained when the diagonal of the square contains 10 parts of which the diameter of the circle contains 8 . Thus, $\pi \simeq 31 / 8$.

1525. Albrecht Dürer (1471-1528) was a famous artist and mathematician. His book Underweysung der Messung mit dem Zirckel und Richtscheyt provides measurement of lines, areas and solids by means of compass and ruler, particularly there is a discussion of squaring the circle.

1544. Oronce Fińe (1494-1555) was a prolific author of mathematical books. He was imprisoned in 1524, probably for practicing judicial astrology. He approximated $\pi$ as $311 / 63=3.174603 \ldots$ Later, he gave $32 / 15=3.133333 \ldots$ and, in $1556,311 / 78=$ $3.141025 \ldots$

1559. Johannes Buteo (1492-1572), a French scholar published a book De quadratura circuli, which seems to be the first book that accounts the history of $\pi$ and related problems. 
1573. Valentin Otho (around 1550-1603) was a German mathematician and astronomer. In 1573, he came to Wittenberg and proposed to Johannes Praetorius the Tsu Ch'ung-chih approximate value of $\pi$ as $355 / 113$.

1580. Tycho Brahe was an astronomer and an alchemist and was known for his most accurate astronomical and planetary observations of his time. His data was used by his assistant, Kepler, to derive the laws of planetary motion. He observed a new star in 1572 and a comet in 1577. In 1566, when he was just 20, he lost his nose partially in a duel with another student in Wittenberg and wore throughout his life a metal insert over his nose. His approximation to $\pi$ is $88 / \sqrt{785}=3.140854 \ldots$.

1583. Simon Duchesne finds $\pi=(39 / 22)^{2}=3.142561 \ldots$.

About 1584. Zhu Zaiyu (1536-1611), a noted musician, mathematician and astronomercalendarist, Prince of the Ming Dynasty, obtained the twelfth root of two. He also gave the approximate value of $\pi$ as $\sqrt{2} / 0.45=3.142696 \ldots$. Around the same time Xing Yunlu adopted $\pi$ as 3.1126 and 3.12132034, while Chen Jinmo and Fang Yizhi, respectively, took as 3.1525 and $52 / 17$.

1584. Simon van der Eycke (Netherland) published an incorrect proof of the quadrature of the circle. He approximated $\pi$ as $1,521 / 484=3.142561 \ldots$. In 1585 , he gave the value 3.1416055 .

1585. Adriaen Anthoniszoon (1529-1609) was a mathematician and fortification engineer. He rediscovered the Tsu Ch'ung-chih approximation 355/113 to $\pi$. This was apparently lucky incident, since all he showed was that $377 / 120>\pi>333 / 106$. He then averaged the numerators and the denominators to obtain the 'exact' value of $\pi$.

1593. Francois Viéte (1540-1603) is frequently called by his semi-Latin name of Vieta. In relation to the three famous problems of antiquity, he showed that the trisection of an angle and the duplication of a cube problems depend upon the solution of cubic equations. He has been called the father of modern algebra and the foremost mathematician of the sixteenth century. In his 1593 book, Supplementum geometriae, he showed $3.1415926535<\pi<3.1415926537$, i.e., gave the value of $\pi$ correct to 9 places. For this, he used the classical polygon of $6 \times 2^{16}=393,216$ sides. He also represented $\pi$ as an infinite product

$$
\frac{2}{\pi}=\cos \frac{\pi}{4} \cos \frac{\pi}{8} \cos \frac{\pi}{16} \cos \frac{\pi}{32} \cdots=\frac{\sqrt{2}}{2} \frac{\sqrt{(2+\sqrt{2})}}{2} \frac{\sqrt{(2+\sqrt{(2+\sqrt{2})})}}{2} \cdots .
$$

For this, we note that

$$
\sin x=\cos \frac{x}{2} \cdot 2 \sin \frac{x}{2}=\cos \frac{x}{2} \cos \frac{x}{2^{2}} \cdot 2^{2} \sin \frac{x}{2^{2}}=\cdots=\left(\prod_{k=1}^{n} \cos \frac{x}{2^{k}}\right) 2^{k} \sin \frac{x}{2^{k}}
$$

and hence

$$
\frac{\sin x}{x}=\left(\prod_{k=1}^{n} \cos \frac{x}{2^{k}}\right) \frac{\sin x / 2^{k}}{x / 2^{k}},
$$

which as $k \rightarrow \infty$, and then $x=\pi / 2$ gives

$$
\frac{2}{\pi}=\cos \frac{\pi}{4} \cos \frac{\pi}{8} \cos \frac{\pi}{16} \cos \frac{\pi}{32} \cdots
$$


Finally, note that

$$
\begin{aligned}
& \cos \frac{\pi}{4}=\sqrt{\frac{1}{2}\left(1+\cos \frac{\pi}{2}\right)}=\frac{\sqrt{2}}{2}, \\
& \cos \frac{\pi}{8}=\sqrt{\frac{1}{2}\left(1+\cos \frac{\pi}{4}\right)}=\sqrt{\frac{1}{2}\left(1+\frac{\sqrt{2}}{2}\right)}=\frac{1}{2} \sqrt{2+\sqrt{2}}, \ldots .
\end{aligned}
$$

The above formula (6) is one of the milestones in the history of $\pi$. The convergence of Vieta's formula was proved by Ferdinand Rudio (1856-1929) in 1891. It is clear that Vieta's formula cannot be used for the numerical computation of $\pi$. In fact, the square roots are much too cumbersome, and the convergence is rather slow. It is clear that if we define $a_{1}=\sqrt{1 / 2}$ and $a_{n+1}=\sqrt{\left(1+a_{n}\right) / 2}$, then (6) is the same as $a_{1} a_{2} a_{3} \cdots=2 / \pi$.

1593. Adrianus van Roomen (1561-1615), more commonly referred to as Adrianus Romanus, successively professor of medicine and mathematics in Louvain, professor of mathematics at Würzburg, and royal mathematician (astrologer) in Poland, proposed a challenge to all contemporary mathematicians, to solve a certain 45th degree equation. The Dutch ambassador presented van Roomen's book to King Henry IV with the comment that at present there is no mathematician in France capable of solving this equation. The King summoned and showed the equation to Vieta, who immediately found one solution to the equation, and then the next day presented 22 more. However, negative roots escaped him. In return, Vieta challenged van Roomen to solve the problem of Apollonius, to construct a circle tangent to three given circles, but he was unable to obtain a solution using Euclidean geometry. When van Roomen was shown proposer's elegant solution, he immediately traveled to France to meet Vieta, and a warm friendship developed. The same year Rooman used the classical method with $2^{30}$ sides, to approximate $\pi$ to 15 correct decimal places.

1594. Joseph Justus Scaliger (1540-1609) was a religious leader and scholar. He is known for ancient Greek, Roman, Persian, Babylonian, Jewish and Egyptian history. In his work, Cyclometrica elementa duo he claimed that $\pi$ is equal to $\sqrt{10}$.

1596. Ludolph van Ceulen (1539-1610) was a German who emigrated to the Netherlands. He taught Fencing and Mathematics in Delft until 1594, when he moved to Leiden and opened a Fencing School. In 1600, he was appointed to the Engineering School at Leiden, where he spent the remainder of his life teaching Mathematics, Surveying and Fortification. He wrote several books, including Van den Circkel (On The Circle, 1596), in which he published his geometric findings, and the approximate value of $\pi$ correct to 20 decimal places. For this, he reports that he used classical method with $60 \times 2^{33}$, i.e., $515,396,075,520$ sides. This book ends with 'Whoever wants to, can come closer.'

1610. Ludolph van Ceulen (1539-1610) in his work De Arithmetische en Geometrische fondamenten, which was published posthumously by his wife in 1615 , computed $\pi$ correct to 35 decimal places by using classical method with $2^{62}$ sides. This computational feat was considered so extraordinary that his widow had all 35 digits of die Ludolphsche Zahl (the Ludolphine number) was engraved on his tombstone in St. Peter's churchyard in Leiden. The tombstone was later lost but was restored in 2000. This was one of the last major attempts to evaluate $\pi$ by the classical method; thereafter, the techniques of calculus were employed. 
1621. Willebrord Snell (Snellius) (1580-1626) was a Dutch astronomer and mathematician. At the age of 12 , he is said to have been acquainted with the standard mathematical works, while at the age of 22, he succeeded his father as Professor of Mathematics at Leiden. His fame rests mainly on his discovery in 1621 of the law of refraction, which played a significant role in the development of both calculus and the wave theory of light. However, it is now known that this law was first discovered by Ibn Sahl (940-1000) in 984. Snell cleverly combined Archimedean method with trigonometry, and showed that for each pair of bounds on $\pi$ given by the classical method, considerably closer bounds can be obtained. By his method, he was able to approximate $\pi$ to seven places by using just 96 sides, and to van Ceulen's 35 decimal places by using polygons having only $2^{30}$ sides. The classical method with such polygons yields only two and fifteen decimal places.

1627. Yoshida Mitsuyoshi (1598-1672) was working during Edo period. His 1627 work named as Jinkoki deals with the subject of soroban arithmetic, including square and cube root operations. In this work, he used 3.16 for $\pi$.

1630. Christoph (Christophorus) Grienberger (1561-1636) was an Austrian Jesuit astronomer. The crater Gruemberger on the Moon is named after him. He used Snell's refinement to compute $\pi$ to 39 decimal places. This was the last major attempt to compute $\pi$ by the Archimedes method.

1635. Celiang quanyi (Complete Explanation of Methods of Planimetry and Stereometry) gives without proof the following bounds $3.14159265358979323846<\pi<$ 3.14159265358979323847 , i.e., $\pi$ correct to 19 digits.

1647. William Oughtred (1575-1660), an English mathematician offered free mathematical tuition to pupils, which included even Wallis. His textbook, Clavis Mathematicae (The Key to Mathematics) on arithmetic published in 1631 was used by Wallis and Newton amongst others. In this work, he introduced the $\times$ symbol for multiplication, and the proportion sign (double colon ::). He designated the ratio of the circumference of a circle to its diameter by $\pi / \delta$. His notation was used by Isaac Barrow (1630-1677) a few years later, and David Gregory (1659-1708). Before him, mathematicians described $\pi$ in round-about ways such as 'quantitas, in quam cum multipliectur diameter, proveniet circumferential', which means 'the quantity which, when the diameter is multiplied by it, yields the circumference'.

1647. Grégoire de Saint-Vincent (1584-1667), a Jesuit, was a mathematician who discovered that the area under the hyperbola $(x y=k)$ is the same over $[a, b]$ as over $[c, d]$ when $a / b=c / d$. This discovery played an important role in the development of the theory of logarithms and an eventual recognition of the natural logarithm. In 1668, Nicolaus Mercator (Kauffmann) (1620-1687) wrote a treatise entitled Logarithmo-technica, and discovered the series

$$
\ln (1+x)=x-\frac{1}{2} x^{2}+\frac{1}{3} x^{3}-\frac{1}{4} x^{4}+\cdots
$$

however, the same series was independently discovered earlier by Saint-Vincent. In his book, Opus geometricum quadraturae circuli et sectionum coni he proposed at least four methods of squaring the circle, but none of them were implemented. The fallacy in his quadrature was pointed out by Huygens.

1650. René Descartes (1596-1650) was a thoughtful child who asked so many questions that his father called him 'my little philosopher'. In 1638, he published his Discourse 
on Method, which contained important mathematical work, and three essays, Meteors, Dioptrics and Geometry, produced an immense sensation and his name became known throughout Europe. The rectangular coordinate system is credited to Descartes. He is regarded as a genius of the first magnitude. He was one of the most important and influential thinkers in human history and is sometimes called the founder of modern philosophy. After his death, a novel geometric approach to approximate $\pi$ was found in his papers. His method consisted of doubling the number of sides of regular polygons while keeping the perimeter constant. In modern terms, Descartes' method can be summarized as

$$
\pi=\lim _{k \rightarrow \infty} 2^{k} \tan \left(\frac{\pi}{2^{k}}\right)
$$

If we let $a_{k}=2^{k} \tan \left(\pi / 2^{k}\right), k \geq 2$, then in view of $\tan 2 \theta=2 \tan \theta /\left(1-\tan ^{2} \theta\right), x_{k}=1 / a_{k}$ satisfies the relation

$$
x_{k+1}\left(x_{k+1}-x_{k}\right)=2^{-2 k-2} \text {, }
$$

and hence

$$
x_{k+1}=\frac{1}{2}\left(x_{k}+\left(x_{k}^{2}+2^{-2 k}\right)^{1 / 2}\right), \quad k \geq 2, \quad x_{2}=1 / 4 .
$$

The sequence $\left\{x_{k}\right\}$ generated by the above recurrence relation converges to $1 / \pi$.

1650. John Wallis (1616-1703) in 1649 was appointed as Savilian professor of geometry at the University of Oxford, which he continued for over 50 years until his death. He was the most influential English mathematician before Newton. In his most famous work, Arithmetica infinitorum, which he published in 1656, he established the formula

$$
\pi=2 \cdot \frac{2}{1} \cdot \frac{2}{3} \cdot \frac{4}{3} \cdot \frac{4}{5} \cdot \frac{6}{5} \cdot \frac{6}{7} \cdot \frac{8}{7} \cdot \frac{8}{9} \cdots
$$

This formula is a great milestone in the history of $\pi$. Like Viéte's formula (6), Wallis had found $\pi$ in the form of an infinite product, but he was the first in history whose infinite sequence involved only rational operations. In his Opera Mathematica I (1695), Wallis introduced the term continued fraction. He rejected as absurd the now usual idea of a negative number as being less than nothing, but accepted the view that it is something greater than infinity, specially showed that $-1>\infty$. He had great ability to do mental calculations. He slept badly and often did mental calculations as he lay awake in his bed. On 22 December 1669, he when in bed, occupied himself in finding the integral part of the square root of $3 \times 10^{40}$; and several hours afterward wrote down the result from memory. Two months later, he was challenged to extract the square root of a number of 53 digits; this he performed mentally, and a month later he dictated the answer which he had not meantime committed to writing. Wallis' life was embittered by quarrels with his contemporaries including Huygens, Descartes, and the political philosopher Hobbes, which continued for over 20 years, ending only with Hobbes' death. Hobbes called Arithmetica infinitorum 'a scab of symbols', and claimed to have squared the circle. It seems that to some, individual's quarrels give strength, encouragement and mental satisfaction. To derive (8), we note that 
$I_{n}=\int_{0}^{\pi / 2} \sin ^{n} x d x$ satisfies the recurrence relation

$$
I_{n}=\frac{n-1}{n} I_{n-2}
$$

Thus, in view of $I_{0}=\pi / 2$ and $I_{1}=1$, we have

$$
I_{2 m}=\frac{2 m-1}{2 m} \cdot \frac{2 m-3}{2 m-2} \cdots \frac{5}{6} \cdot \frac{3}{4} \cdot \frac{1}{2} \cdot \frac{\pi}{2}
$$

and

$$
I_{2 m+1}=\frac{2 m}{2 m+1} \cdot \frac{2 m-2}{2 m-1} \cdots \frac{6}{7} \cdot \frac{4}{5} \cdot \frac{2}{3} .
$$

From these relations, a termwise division leads to

$$
\frac{\pi}{2}=\left(\frac{2 \cdot 4 \cdot 6 \cdots 2 m}{3 \cdot 5 \cdots(2 m-1)}\right)^{2} \frac{1}{2 m+1} \frac{I_{2 m}}{I_{2 m+1}} .
$$

Now, it suffices to show that

$$
\lim _{m \rightarrow \infty} \frac{I_{2 m}}{I_{2 m+1}}=1
$$

We know that for all $x \in(0, \pi / 2)$ the inequalities $\sin ^{2 m-1} x>\sin ^{2 m} x>\sin ^{2 m+1} x$ hold. Thus, an integration from 0 to $\pi / 2$ gives $I_{2 m-1} \geq I_{2 m} \geq I_{2 m+1}$, and hence

$$
\frac{I_{2 m-1}}{I_{2 m+1}} \geq \frac{I_{2 m}}{I_{2 m+1}} \geq 1
$$

Further, from (9), we have

$$
\frac{I_{2 m-1}}{I_{2 m+1}}=\frac{2 m+1}{2 m}
$$

thus, it follows that

$$
\lim _{m \rightarrow \infty} \frac{I_{2 m-1}}{I_{2 m+1}}=\lim _{m \rightarrow \infty} \frac{2 m+1}{2 m}=1 .
$$

Finally, a combination of (11) and (12) immediately gives (10). If we define $a_{n}=1-1 /(2 n)^{2}$, then (8) is equivalent to $a_{1} a_{2} a_{3} \cdots=2 / \pi$. We also note that

$$
\frac{1}{a_{1} a_{2} \cdots a_{n}}=\frac{\pi}{2}+O\left(\frac{1}{n}\right)
$$

1650. William Brouncker, 2nd Viscount Brouncker (1620-1684) was one of the founders and the second President of the Royal Society. His mathematical contributions are: reproduction of Brahmagupta's solution of a certain indeterminate equation, calculations of the lengths of the parabola and cycloid, quadrature of the hyperbola which required approximation of the natural logarithm function by infinite series and the study of generalized 
continued fractions. He undertook some calculations to verify formula (8), and showed that $3.141592653569 \ldots<\pi<3.141592653696 \ldots$, which is very satisfactory. He also converted Wallis' result (8) into the continued fraction

$$
\pi=\frac{4}{1+\frac{1}{2+\frac{9}{2+\frac{25}{2+\cdots}}}} .
$$

Neither of the expressions (8), and (13); however, later has served for an extensive calculation of $\pi$.

Another continued fraction representation of $\pi$ which follows from the series (5) is

$$
\pi=\frac{4}{1+\frac{1^{2}}{3+\frac{2^{2}}{5+\frac{3^{2}}{7+\cdots}}}} .
$$

1654. Christiaan Huygens (1629-1695) is famous for his invention of the pendulum clock, which was a breakthrough in timekeeping. He formulated the second law of motion of Newton in a quadratic form, and derived the now well-known formula for the centripetal force, exerted by an object describing a circular motion. Huygens was the first to derive the formula for the period of an ideal mathematical pendulum (with massless rod or cord), $T=2 \pi \sqrt{\ell / g}$. For the computation of $\pi$, he gave the correct proof of Snell's refinement, and using an inscribed polygon of only 60 sides obtained the bounds $3.1415926533<\pi<3.1415926538$, for the same accuracy the classical method requires almost 400,000 sides.

1663. Muramatsu Shigekiyo (1608-1695) published Sanso, or Stack of Mathematics, in which he used classical polygon method of $2^{15}$ sides to obtain $\pi=3.14195264877$.

1665. Sir Isaac Newton (1642-1727), hailed as one of the greatest scientist-mathematicians of the English-speaking world, had the following more modest view of his own monumental achievements: '.. to myself I seem to have been only like a boy playing on the seashore, and diverting myself in now and then finding a smoother pebble or a prettier shell than ordinary, whilst the great ocean of truth lay all undiscovered before me'. As he examined these shells, he discovered to his amazement more and more of the intricacies and beauties that lay in them, which otherwise would remain locked to the outside world. At the age of 26, he succeeded Barrow as Lucasian professor of mathematics at Cambridge. About him, Aldous Huxley (1894-1963) had said 'If we evolved a race of Isaac Newtons, that would not be progress. For the price Newton had to pay for being a supreme intellect was that he was incapable of friendship, love, fatherhood and many other desirable things. As a man he was a failure; as a monster he was superb. Newton made some of the greatest discoveries the world ever knew at that time. Newton discovered: 1 . The nature of colors. 2. The law of gravitation and the laws of mechanics. 3. The fluxional calculus. Most of the history books say that to compute $\pi$ Newton used the series

$$
\sin ^{-1} x=x+\frac{1 \cdot x^{3}}{2 \cdot 3}+\frac{1 \cdot 3 \cdot x^{5}}{2 \cdot 4 \cdot 5}+\frac{1 \cdot 3 \cdot 5 \cdot x^{7}}{2 \cdot 4 \cdot 6 \cdot 7}+\cdots,
$$


Figure 2 Half-circle.

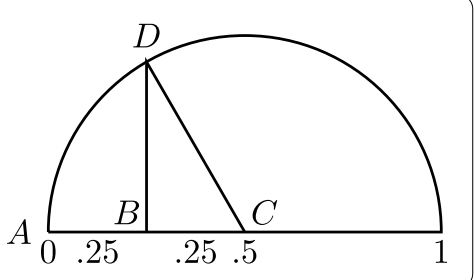

which for $x=1 / 2$ gives

$$
\frac{\pi}{6}=\sin ^{-1}\left(\frac{1}{2}\right)=\left(\frac{1}{2}+\frac{1}{2 \cdot 3 \cdot 2^{3}}+\frac{1 \cdot 3}{2 \cdot 4 \cdot 5 \cdot 2^{5}}+\cdots\right)
$$

however, he actually used twenty-two terms to obtain 16 decimal places of the following series

$$
\pi=\frac{3 \sqrt{3}}{4}+24\left(\frac{1}{3 \cdot 2^{2}}-\frac{1}{5 \cdot 2^{5}}-\frac{1}{28 \cdot 2^{7}}-\frac{1}{72 \cdot 2^{9}}-\cdots\right) .
$$

Later, he wrote 'I am ashamed to tell you to how many figures I carried these computations, having no other business at the time'. His result was not published until 1737 (posthumously).

Using analysis and geometry, the series (14) can be obtained as follows: From Figure 2, the equation of the upper half circle is $y=x^{1 / 2}(1-x)^{1 / 2}$. Thus, binomial expansion gives

$$
y=x^{1 / 2}-\frac{1}{2} x^{3 / 2}-\frac{1}{8} x^{5 / 2}-\frac{1}{16} x^{7 / 2}-\frac{5}{128} x^{9 / 2}-\frac{7}{256} x^{11 / 2}-\cdots
$$

Thus, the area of the sector $A B D$ is (integrating the above series from 0 to $1 / 4$ )

$$
\triangle A B D=\frac{1}{3 \cdot 2^{2}}-\frac{1}{5 \cdot 2^{5}}-\frac{1}{28 \cdot 2^{7}}-\frac{1}{72 \cdot 2^{9}}-\cdots
$$

Also, from geometry the area of the sector $A B D$ is

$$
\begin{aligned}
\triangle A B D & =\triangle A B C D-\triangle B C D \\
& =\frac{1}{3}\left(\frac{1}{2} \pi\left(\frac{1}{2}\right)^{2}\right)-\frac{1}{2}\left(\frac{1}{4}\right) \sqrt{\left(\frac{1}{2}\right)^{2}-\left(\frac{1}{4}\right)^{2}}=\frac{\pi}{24}-\frac{\sqrt{3}}{32} .
\end{aligned}
$$

Equating (15) and (16), we immediately get (14).

1666. Thomas Hobbes of Malmesbury (1588-1679) was an English philosopher, best known today for his work on political philosophy. He also contributed in several other diverse fields, including history, geometry, the physics of gases, theology, ethics and general philosophy. He approximated $\pi$ by $31 / 5=3.2$, which was refuted by Huygens and Wallis. In 1678 , he also gave the approximation $\sqrt{10}$.

1671. James Gregory (1638-1675) published two books Vera circuli et hyperbolae quadratura in 1667, and Geometriae pars universalis in 1668. In the first book particularly, he showed that the area of a circle can be obtained in the form of an infinite convergent series only, and hence inferred that the quadrature of the circle was impossible. 
In the second book, he attempted to write calculus systematically, which perhaps made the basis of Newton's fluxions. This book also contains series expansions of $\sin (x), \cos (x)$, $\arcsin (x)$ and $\arccos (x)$; however, as we have seen earlier these expansions were known to Madhava. Gregory anticipated Newton in discovering both the interpolation formula and the general binomial theorem as early as 1670. In early 1671, he discovered Taylor's theorem (published by Taylor in 1715); however, he did not publish. Later in 1671, he rediscovered Nilakanthan's arctangent series (5). In his Vera circuli et hyperbolae quadratura of 1667 , Gregory tried to show that $\pi$ was a transcendental number, but his attempt, though very interesting, was not successful. Huygens made detailed and rather biased criticisms of it.

1672. Pietro Mengoli (1626-1686) studied at the University of Bologna, and became a professor there in 1647 for the next 39 years of his life. Besides proposing Basel problem, he proved that the harmonic series does not converge, established that the alternating harmonic series is equal to the natural logarithm of 2, published on the problem of squaring the circle, and provided a proof that Wallis' product (8) for $\pi$ is correct.

1674. Gottfried Wilhelm von Leibniz (1646-1716) was a universal genius who won recognition in many fields - law, philosophy, religion, literature, politics, geology, metaphysics, alchemy, history and mathematics. He shares credit with Newton in developing calculus independently. He popularized and gave several mathematical symbols. Leibniz tried to reunite the Protestant and Catholic churches. He in binary arithmetic saw the image of Creation. He imagined that Unity represented God, and Zero the void; that the Supreme Being drew all beings from the void, just as unity and zero express all numbers in the binary system of numeration. He communicated his idea to the Jesuit Grimaldi, who was the President of the Chinese tribunal for mathematics in the hopes that it would help convert to Christianity the Emperor of China, who was said to be very fond of the Sciences. Later Leibniz became an expert in the Sanskrit language and the culture of China. For calculating $\pi$, he developed a method without any reference to a circle. In 1674, he also rediscovered Nilakanthan's arctangent series (5), whose beauty he described by saying that Lord loves odd numbers. Leibniz even invented a calculating machine that could perform the four operations and extract roots.

1684. Isomura Yoshinori (1640-1710) employed a $2^{17}$-sided inscribed polygon to obtain 3.141592664 for $\pi$, but for some reason he wrote only $\pi=3.1416$.

1685. Father Adam Adamad Kochansky (1631-1700) was librarian of the Polish King John III. He was the first to utilize a steel spring for suspension of the pendulum of a clock. He used a new approximate geometric construction for $\pi$ to obtain

$$
\pi \simeq \sqrt{\frac{40}{3}-2 \sqrt{3}}=3.141533 \ldots
$$

His method was later quoted in several geometrical textbooks.

1690. Takebe Katahiro (1664-1739) also known as Takebe Kenko played a critical role in the development of a crude version of the calculus. He also created charts for trigonometric functions. He used polygon (just 1,024 sides) approximation and a numerical method which is essentially equivalent to the Romberg algorithm (rediscovered by Sigmund Romberg, 1887-1951) to compute $\pi$ to 41 digits. In 1722, Takebe obtained power series expansion of $\left(\sin ^{-1} x\right)^{2}, 15$ years earlier than Euler. Around 1729, essentially the same 
series was rediscovered by Oyama Shokei who used it to find the expansion

$$
\pi^{2}=8\left(1+\sum_{n=1}^{\infty} \frac{2^{n+1}(n !)^{2}}{(2 n+2) !}\right)=8\left(1+\frac{1}{2} \cdot \frac{1}{3}+\frac{1}{3} \cdot \frac{1 \cdot 2}{3 \cdot 5}+\frac{1}{4} \cdot \frac{1 \cdot 2 \cdot 3}{3 \cdot 5 \cdot 7}+\cdots\right) .
$$

The above expansion of $\pi^{2}$ was also given by Yamaji Nushizumi (1704-1772) around 1765.

1699. Abraham Sharp (1653-1742) was a mathematician and astronomer. In 1688, he joined the Greenwich Royal Observatory and did notable work, improving instruments and showing great skill as a calculator. He also worked on geometry and improved logarithmic tables. In the supervision of Edmund Halley (1656-1742), he realized that by putting $x=1$ in (4) (see (5)) we lose the benefit of the powers $x^{3}, x^{5}, x^{7}, \ldots$, which tend to increase the rapidity of convergence for smaller values of $x$. He substituted $x=1 / \sqrt{3}$ in (4), to obtain

$$
\frac{\pi}{6}=\frac{1}{\sqrt{3}}\left(1-\frac{1}{3 \cdot 3}+\frac{1}{3^{2} \cdot 5}-\frac{1}{3^{3} \cdot 7}+\cdots\right) .
$$

Sharp used (17) to calculated $\pi$ to 72 decimal places out of which 71 digits are correct. In (17), the 10 th term is $1 /\left(\sqrt{3} \cdot 19 \cdot 3^{9}\right)$, which is less than 0.00005 , and hence we have at least 4 places correct after just 9 terms. It is believed that Madhava of Sangamagramma used the same series in the fourteenth century to compute the value of $\pi$ correct to 11 decimal places.

1700. Seki Takakazu also known as Seki Kowa (1642-1708) is generally regarded as the greatest Japanese mathematician. He was a prolific writer, and a number of his publications are either transcripts of mathematics from Chinese into Japanese, or commentaries on certain works of well-known Chinese mathematicians. His interests in mathematics ranged recreational mathematics, magic squares and magic circles, solutions of higherorder and indeterminate equations, conditions for the existence of positive and negative roots of polynomials, and continued fractions. He discovered determinants ten years before Leibniz, and the Bernoulli numbers a year before Bernoulli. He used polygon of $2^{17}$ sides and Richardson extrapolation (rediscovered by Alexander Craig Aitken, 1895-1967) to compute $\pi$ to 10 digits. Some authors believe that he also used the formula

$$
\pi=\lim _{n \rightarrow \infty} \frac{4}{n^{2}} \sum_{j=0}^{n} \sqrt{n^{2}-j^{2}}
$$

About 1700. Oliver de Serres believed that by weighing a circle and a triangle equal to the equilateral triangle inscribed he had found that the circle was exactly double of the triangle, not being aware that this double is exactly the hexagon inscribed in the same circle. Thus, according to him $\pi=3$.

1706. William Jones (1675-1749), an obscure English writer, represented the ratio of the circumference of a circle to its diameter by $\pi$ in his Synopsis Palmariorum Matheseos (New Introduction to the Mathematics). He used the letter $\pi$ as an abbreviation for the Greek word perimetros (periphery) (of a circle with unit diameter). In his book, he published the value of $\pi$ correct to 100 decimal places.

1706. John Machin (1680-1752) was a professor of astronomy at Gresham College, London. He also served as secretary of the Royal Society during 1718-1747. Machin is best 
remembered for computing the value of $\pi$ to 100 decimal places by using the formula

$$
\frac{\pi}{4}=4 \tan ^{-1}\left(\frac{1}{5}\right)-\tan ^{-1}\left(\frac{1}{239}\right)
$$

which in view of (4) is the same as

$$
\frac{\pi}{4}=4\left(\frac{1}{5}-\frac{1}{3 \cdot 5^{3}}+\frac{1}{5 \cdot 5^{5}}-\cdots\right)-\left(\frac{1}{239}-\frac{1}{3 \cdot 239^{3}}+\frac{1}{5 \cdot 239^{5}}-\cdots\right) .
$$

To establish (18), we let $\tan \theta=1 / 5$, so that

$$
\tan 2 \theta=\frac{2 \tan \theta}{1-\tan ^{2} \theta}=\frac{5}{12} \text { and } \tan 4 \theta=\frac{2 \tan 2 \theta}{1-\tan ^{2} 2 \theta}=\frac{120}{119} .
$$

Thus, it follows that

$$
\tan \left(4 \theta-\frac{\pi}{4}\right)=\frac{\tan 4 \theta-1}{1+\tan 4 \theta}=\frac{1}{239}
$$

and hence

$$
\tan ^{-1}\left(\frac{1}{239}\right)=4 \theta-\frac{\pi}{4}=4 \tan ^{-1}\left(\frac{1}{5}\right)-\frac{\pi}{4} .
$$

The proof of (18) also follows by comparing the angles in the identity (the idea originally goes back to Caspar Wessel (1745-1818) who presented his work in 1797 to the Royal Danish Academy of Sciences)

$$
(5+i)^{4}(-239+i)=-114,244(1+i)
$$

i.e.,

$$
4 \tan ^{-1}\left(\frac{1}{5}\right)+\pi-\tan ^{-1}\left(\frac{1}{239}\right)=\pi+\tan ^{-1} 1
$$

The series (19) certainly converges significantly faster than (5) and (17). In fact, taking six terms of the first series and two terms of the second and paying attention to the remainders and round-off errors, we get the inequalities $3.141592629<\pi<3.141592668$. Thus, the value of $\pi$ correct to seven decimals is 3.1415926 .

Several other Machin-type formulas are known, e.g.,

$$
\begin{aligned}
\frac{\pi}{4} & =2 \tan ^{-1}\left(\frac{1}{2}\right)-\tan ^{-1}\left(\frac{1}{7}\right) \\
& =5 \tan ^{-1}\left(\frac{1}{5}\right)-3 \tan ^{-1}\left(\frac{1}{18}\right)-2 \tan ^{-1}\left(\frac{1}{57}\right) \\
& =17 \tan ^{-1}\left(\frac{1}{22}\right)+3 \tan ^{-1}\left(\frac{1}{172}\right)-2 \tan ^{-1}\left(\frac{1}{682}\right)-7 \tan ^{-1}\left(\frac{1}{5,357}\right) .
\end{aligned}
$$

For a long list of such type of formulas with a discussion of their relative merits in computational work, see Lehmer (1938). 
1713. Chapter 15 of Shu li jing yun (Collected Basic Principles of Mathematics), which was commissioned by the Emperor Kang Xi and edited by Mei Gucheng and He Guozong, gives $\pi=3.14159265$, which is correct to eight decimal places.

1719. Thomas Fantet de Lagny (1660-1734) was a French mathematician who is well known for his contributions to computational mathematics. He used the series (17) to determine the value of $\pi$ up to 127 decimal places; however, only 112 are correct.

1728. Alexander Pope (1688-1744) was an English poet. He is the third-most frequently cited writer in The Oxford Dictionary of Quotations, after Shakespeare and Tennyson. In his Dunciad it is mentioned that 'The mad Mathesis, now, running round the circle, finds it square.' This explains the wild and fruitless attempts of squaring the circle.

1728. Sieur Malthulon (France) offered solutions to squaring the circle and to perpetual motion. He offered 1,000 crowns reward in legal form to anyone proving him wrong. Nicoli, who proved him wrong, collected the reward and abandoned it to the Hotel Dieu of Lyons. Later, the courts gave the money to the poor.

1730. Toshikiyo Kamata (1678-1747) used both the circumscribed and inscribed polygons and gave the bounds 3.14159265358979323846265341667< < < 3.14159265358979323846264336658 .

1730. Abraham De Moivre (1667-1754) was an intimate personal friend of Newton, and was elected an FRS of London in 1697. In 1710, he was appointed to the Commission set up by the Royal Society to solve the Newton-Leibniz dispute concerning which of them invented calculus first. He is best known for his memoir Doctrine of Chances: A method of calculating the probabilities of events in play, which was first printed in 1618 and dedicated to Newton. In 1722, he published his famous theorem $(\cos x+i \sin x)^{n}=\cos n x+\sin n x$. In his Miscellanea Analytica published in 1730, appears the formula for very large $n$,

$$
n ! \simeq(2 \pi n)^{1 / 2} e^{-n} n^{n}
$$

which is known today as Stirling's formula. In 1733, De Moivre used this formula to derive the normal curve as an approximation to the binomial.

1735. Leonhard Euler (1707-1783) was probably the most prolific mathematician who ever lived. He was born in Basel (Switzerland), and had the good fortune to be tutored one day a week in mathematics by a distinguished mathematician, Johann Bernoulli (16671748). Euler's energy and capacity for work were virtually boundless. His collected works form about 60 to 80 quarto-sized volumes and it is believed that much of his work has been lost. What is particularly astonishing is that Euler became virtually sightless in his right eye during the mid-1730s, and was blind for the last 17 years of his life, and this was one of the most productive periods. In 1644, Mengoli asked for the precise summation of the infinite series $\sum_{n=1}^{\infty} n^{-2}$. The series is approximately equal to $1.644934 \ldots$ In the literature, this problem has been referred after Basel, hometown of Euler as well as of the Bernoulli family who unsuccessfully attacked the problem. Basel problem appears in number theory, e.g., if two positive integers are selected at random and independently of each other, then the probability that they are relatively prime is $\left(\sum_{n=1}^{\infty} n^{-2}\right)^{-1}$ (R. Chartres, 1904). An integer that is not divisible by the square of any prime number is said to be square free. The probability that a randomly selected integer is square free is also $\left(\sum_{n=1}^{\infty} n^{-2}\right)^{-1}$. Euler considered the function $\sin x / x, x \neq 0$ which has the roots at $\pm n \pi, n=1,2, \ldots$. Thus, it 
follows that

$$
\begin{aligned}
\frac{\sin x}{x} & =1-\frac{x^{2}}{3 !}+\frac{x^{4}}{5 !}-\frac{x^{6}}{7 !}+\cdots \\
& =\left(1-\frac{x^{2}}{1^{2} \pi^{2}}\right)\left(1-\frac{x^{2}}{2^{2} \pi^{2}}\right)\left(1-\frac{x^{2}}{3^{2} \pi^{2}}\right) \cdots
\end{aligned}
$$

Thus, on equating the coefficients of $x^{2}$, we get

$$
\frac{1}{6}=\frac{1}{1^{2} \pi^{2}}+\frac{1}{2^{2} \pi^{2}}+\frac{1}{3^{2} \pi^{2}}+\cdots,
$$

which is the same as

$$
\frac{1}{1^{2}}+\frac{1}{2^{2}}+\frac{1}{3^{2}}+\cdots=\frac{\pi^{2}}{6}
$$

The above proof of Euler is based on manipulations that were not justified at the time, and it was not until 1741 that he was able to produce a truly rigorous proof. It is interesting to note that (20) with $x=\pi / 2$ immediately gives Wallis' formula (8). Today, several different proofs of (21) are known in the literature. Euler also established the following series:

$$
\begin{aligned}
& 3 \sum_{m=1}^{\infty} \frac{1}{m^{2}\left(\begin{array}{c}
2 m \\
m
\end{array}\right)}=\frac{\pi^{2}}{6}, \\
& \frac{1}{1^{2}}+\frac{1}{3^{2}}+\frac{1}{5^{2}}+\cdots=\frac{\pi^{2}}{8}, \\
& \frac{1}{1^{2}}-\frac{1}{2^{2}}+\frac{1}{3^{2}}-\frac{1}{4^{2}}+\cdots=\frac{\pi^{2}}{12} .
\end{aligned}
$$

Later, Euler generalized the Basel problem considerably, in fact, for all positive integers $k=1,2, \ldots$, he established the series

$$
\frac{1}{1^{2 k}}+\frac{1}{2^{2 k}}+\frac{1}{3^{2 k}}+\cdots=\frac{(2 \pi)^{2 k}(-1)^{n+1} B_{2 k}}{2(2 n) !},
$$

where $B_{2 k}$ are Bernoulli numbers: $B_{2}=1 / 6, B_{4}=-1 / 30, B_{6}=1 / 42, B_{8}=-1 / 30, B_{10}=$ $5 / 66, \ldots$. In particular, he established

$$
\frac{1}{1^{26}}+\frac{1}{2^{26}}+\frac{1}{3^{26}}+\cdots=\frac{2^{24} \times 76,977,927}{27 !} \pi^{26}
$$

Euler's ideas were taken up years later by George Friedrich Bernhard Riemann (1826-1866) in his seminal 1859 paper, On the Number of Primes Less Than a Given Magnitude, in which he defined his zeta function

$$
\zeta(s)=1+\frac{1}{2^{s}}+\frac{1}{3^{s}}+\frac{1}{4^{s}}+\cdots, \quad s=\sigma+i t,
$$

and proved its basic properties, and in a sovereign way simply stated a number of others without proof. After his death, many of the finest mathematicians of the world have exerted their strongest efforts and created new branches of analysis in attempts to prove 
these statements. Since then with one exception, every statement has been settled in the sense Riemann expected. This exception is the famous Riemann hypothesis: that all the zeros of $\zeta(s)$ in the strip $0 \leq \sigma \leq 1$ lie on the central line $\sigma=1 / 2$. It stands today as the most important unsolved problem of mathematics, and perhaps the most difficult problem that the mind of man has ever conceived.

1737. The letter $\pi$ was first used by Euler in 1737 in his Variae observationes circa series infinitas. Until that time, he had been using the letters $p$ (1734), or $c$ (1736). In 1742, Christian Goldbach (1690-1764) also used $\pi$. After the publication of Euler's treatise: Introductio in Analysin Infinitorum (1748), $\pi$ became a standard symbol, as was the case with other notations he adopted. In 1737, Euler also showed that both $e$ and $e^{2}$ are irrational and gave several continued fractions for $e$. In another paper, De variis modis circuli quadraturam numeris proxime exprimendi of 1737, Euler derived the formulas

$$
\tan ^{-1}\left(\frac{1}{p}\right)=\tan ^{-1}\left(\frac{1}{p+q}\right)+\tan ^{-1}\left(\frac{q}{p^{2}+p q+1}\right)
$$

and

$$
\tan ^{-1}\left(\frac{x}{y}\right)=\tan ^{-1}\left(\frac{a x-y}{a y+x}\right)+\tan ^{-1}\left(\frac{b-a}{a b+1}\right)+\tan ^{-1}\left(\frac{c-b}{c b+1}\right)+\cdots
$$

and these give rise to any amount of relations for $\pi$; for example, if $x=1=y$, and the odd numbers are substituted for $a, b, c, \ldots$, we obtain

$$
\frac{\pi}{4}=\tan ^{-1}\left(\frac{1}{2}\right)+\tan ^{-1}\left(\frac{1}{8}\right)+\tan ^{-1}\left(\frac{1}{18}\right)+\cdots .
$$

The proof of (22) immediately follows by comparing the angles in the identity

$$
(p+q+i)\left(p^{2}+p q+1+i q\right)=\left[(p+q)^{2}+1\right](p+i)
$$

1739. Matsunaga Yoshisuke (died in 1844) was a prolific writer. In modern terms, he used the hypergeometric series

$$
F(a, b, c, x)=1+\frac{a b x}{1 ! c}+\frac{a(a+1) b(b+1) x^{2}}{2 ! c(c+1)}+\frac{a(a+1)(a+2) b(b+1)(b+2) x^{3}}{3 ! c(c+1)(c+2)}+\cdots
$$

for $a=1 / 2, b=1 / 2, c=3 / 2$, and $x=1 / 4$, i.e., the series

$$
\pi=3 F\left(\frac{1}{2}, \frac{1}{2}, \frac{3}{2}, \frac{1}{4}\right)=3\left(1+\frac{1^{2}}{4 \cdot 2 \cdot 3}+\frac{3^{2}}{4^{2} \cdot 2 \cdot 3 \cdot 4 \cdot 5}+\frac{3^{2} \cdot 5^{2}}{4^{3} \cdot 2 \cdot 3 \cdot 4 \cdot 5 \cdot 6 \cdot 7}+\cdots\right)
$$

to compute $\pi$ correct to 50 digits. He also gave the following series:

$$
\pi^{2}=9\left(1+\frac{1^{2}}{3 \cdot 4}+\frac{1^{2} \cdot 2^{2}}{3 \cdot 4 \cdot 5 \cdot 6}+\frac{1^{2} \cdot 2^{2} \cdot 3^{2}}{3 \cdot 4 \cdot 5 \cdot 6 \cdot 7 \cdot 8}+\cdots\right) .
$$

1748. The following expansion of $\pi$ is due to Euler

$$
\pi=1+\frac{1}{2}+\frac{1}{3}+\frac{1}{4}-\frac{1}{5}+\frac{1}{6}+\frac{1}{7}+\frac{1}{8}+\frac{1}{9}-\frac{1}{10}+\frac{1}{11}+\frac{1}{12}-\frac{1}{13}+\cdots,
$$


where the signs are determined following the rule: If the denominator is a prime of the form $4 m-1$, the sign is positive; if the denominator is 2 or a prime of the form $4 m+1$, the sign is negative; for composite numbers, the sign is equal to the product of signs of its factors. The following curious infinite product was also given by Euler:

$$
\frac{\pi}{4}=\frac{3}{4} \cdot \frac{5}{4} \cdot \frac{7}{8} \cdot \frac{11}{12} \cdot \frac{13}{12} \cdot \frac{17}{16} \cdot \frac{19}{20} \cdot \frac{23}{24} \cdot \frac{29}{28} \cdot \frac{31}{32} \cdots,
$$

where the numerators are the odd primes and each denominator is the multiple of four nearest to the numerator.

1750. Henry Sullamar, a real Bedlamite, found the quadrature of the circle in the number 666 inscribed on the forehead of the beast in the Revelations. He published periodically every two or three years some pamphlet in which he endeavored to prop his discovery.

1753. M. de Causans of the Guards cut a circular piece of turf, squared it and from the result deduced original sin and the Trinity. He found that the circle was equal to the square in which it is inscribed, i.e., $\pi=4$. He offered a reward for the detection of any error, and actually deposited 10,000 francs as earnest of 300,000. But the courts did not allow any one to recover.

1754. Jean Étienne Montucla (1725-1799) was an early French historian of mathematics. He published an anonymous treatise entitled Histoire des récherches sur la quadrature du cercle, and in 1758 the first part of his great work Histoire des mathématiques.

1755. Euler in his treatise De relatione inter ternas pluresve quantitates instituenda, which was published ten years later, wrote 'It appears to be fairly certain that the periphery of a circle constitutes such a peculiar kind of transcendental quantities that it can in no way be compared with other quantities, either roots or other transcendentals. This conjecture haunted mathematicians for 107 years. The following expansion is due to Euler:

$$
\tan ^{-1} x=\frac{y}{x}\left(1+\frac{2}{3} y+\frac{2 \cdot 4}{3 \cdot 5} y^{2}+\frac{2 \cdot 4 \cdot 6}{3 \cdot 5 \cdot 7} y^{3}+\cdots\right),
$$

where $y=x^{2} /\left(1+x^{2}\right)$. It converges rapidly.

1760. Georges Louis Leclerc (Comte of Buffon 1707-1788) was a naturalist, mathematician, cosmologist and encyclopedic author. Suppose a number of parallel lines, distance $a$ apart, are ruled on a horizontal plane, and suppose a homogeneous uniform rod of length $\ell<a$ is dropped at random onto the plane. Buffon showed that the probability that the rod will fall across one of the lines in the plane is given by $p=(2 \ell / \pi a)$. In the literature, this problem is known as Buffon's needle problem. This was the earliest problem in geometric probability to be solved. By actually performing this experiment, a large number of times and noting the number of successful cases, we can compute an approximation for $\pi$.

1761. Johann Heinrich Lambert (1728-1777) was the first to introduce hyperbolic functions into trigonometry. He wrote landmark books on geometry, the theory of cartography, and perspective in art. He is also credited for expressing Newton's second law of motion in the notation of the differential calculus. Lambert used the properties of continued fractions to show that $\pi$ is irrational. He published a more general result in 1768 . Lambert also showed that the functions $e^{x}$ and $\tan x$ cannot assume rational values if $x$ is 
a non-zero rational number. He also gave an interesting continued fraction for $\pi$,

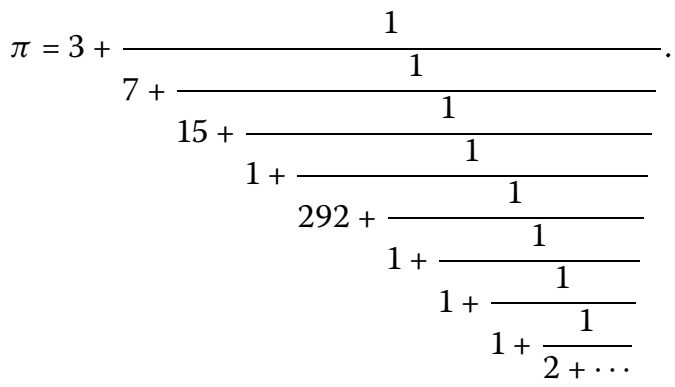

Some inverse convergents of this continued fraction are as follows:

$$
\begin{aligned}
& \frac{3}{1}, \quad \frac{22}{7}, \quad \frac{333}{106}, \quad \frac{355}{113}, \quad \frac{103,993}{33,102}, \quad \frac{104,348}{33,215}, \quad \frac{208,341}{66,317}, \\
& \frac{312,689}{99,532}, \quad \frac{833,719}{265,381}, \quad \frac{1,146,408}{364,913}, \quad \frac{4,272,943}{1,360,120} \text {. }
\end{aligned}
$$

1766. Arima Yoriyuki (1714-1783) was a Japanese mathematician of the Edo period. $\mathrm{He}$ found the following rational approximation of $\pi$, which is correct to 29 digits

$$
\pi=\frac{428,224,593,349,304}{136,308,121,570,117} .
$$

1775. The French Academy of Sciences passed a resolution henceforth not to examine any more solutions of the problem of squaring the circle. In fact, it became necessary to protect its officials against the waste of time and energy involved in examining the efforts of circle squarers. A few years later, the Royal Society in London also banned consideration of any further proofs of squaring the circle. This decision of the Royal Society was described by Augustus De Morgan (1806-1871) about 100 years later as the official blow to circlesquarers.

1776. Charles Hutton (1737-1823) was an English mathematician. He wrote several mathematical texts. In 1774, he was elected a fellow of the Royal Society of London. He suggested Machin's stratagem in the form

$$
\pi=20 \tan ^{-1}\left(\frac{1}{7}\right)+8 \tan ^{-1}\left(\frac{3}{79}\right)
$$

however, he did not carry computations far enough. Euler also developed the formula (24).

1778. M. de Vausenville, one of the deluded individuals, brought an action against the French Academy of Sciences to recover a reward to which he felt himself entitled. It ought to be needless to say that there was no reward offered for squaring the circle.

1779. Euler used his expansion (23) to evaluate right terms of (24), to calculate $\pi$ to 20 decimal places in one hour!

About 1785. Franz Xaver Freiherr von Zach (1754-1832) discovered a manuscript by an unknown author in the Radcliffe Library, Oxford, which gives the correct value of $\pi$ to 152 decimal places. Zach was elected a member of the Royal Swedish Academy of Sciences in 1794, a Fellow of the Royal Society in 1804, and an honorary member of the Hungarian 
Academy of Sciences in 1832. Asteroid 999 Zachia and the crater Zach on the Moon are named after him.

1789. Baron Jurij Bartolomej Vega (Georg Vega 1754-1802) was a Slovene mathematician, physicist and artillery officer. He wrote six scientific papers. The record of de Lagny of 127 digits seems to have stood until 1789, when Vega, using a new series for the arctangent discovered by Euler in 1755, calculated 140 decimal places (126 correct). Vega's result showed that de Lagny's string of digits had a 7 instead of an 8 in the 113th decimal place. His article was not published until six years later, in 1795 (136 correct). Vega retained his record for 52 years until 1841 .

1794. Adrien-Marie Legendre (1752-1833) is remembered for Legendre functions, law of quadratic reciprocity for residues, standardizing weights and measures to the metric system, supervising the major task of producing logarithmic and trigonometric tables, least squares method of fitting a curve to the data available, proof of Fermat's last theorem for the exponent $n=5$, Gauss-Legendre algorithm, Legendre's constant, Legendre's equation, Legendre polynomials, Legendre's conjecture, and Legendre transformation. The Legendre crater on the Moon is named after him. Legendre, in his Elements de Géometrie (1794) used a slightly modified version of Lambert's argument to prove the irrationality of $\pi$ more rigorously, and also gave a proof that $\pi^{2}$ is irrational. He writes: 'It is probable that the number $\pi$ is not even contained among the algebraic irrationalities, i.e., that it cannot be the root of an algebraic equation with a finite number of terms, whose coefficients are rational. But, it seems to be very difficult to prove this strictly'.

1795. Ajima Naonobu (1732-1798), also known as Ajima Chokuyen, was a Japanese mathematician of the Edo period. The series he developed can be simplified as

$$
\begin{aligned}
\frac{\pi}{2} & =F\left(1,1, \frac{3}{2}, \frac{1}{2}\right)=\left(1+\frac{1 !}{3}+\frac{2 !}{3 \cdot 5}+\frac{3 !}{3 \cdot 5 \cdot 7}+\frac{4 !}{3 \cdot 5 \cdot 7 \cdot 9} \cdots\right) \\
& =\sum_{i=0}^{\infty} \frac{i !}{(2 i+1) ! !}=\sum_{i=0}^{\infty} \frac{(i !)^{2} 2^{i}}{(2 i+1) !}
\end{aligned}
$$

It is interesting to note that the above series follows from (5) by using an acceleration technique known in the literature as Euler's transform. It can also be derived from the Wallis product formula (8).

1795. Jean-Charles Callet (174-1799) in his tables gave 154 (152 correct) decimal digits of $\pi$.

1797. Lorenzo Mascheroni (1750-1800) was educated with the aim of becoming a priest and he was ordained at the age of 17. In 1790, he calculated Euler's constant to 32 (19 correct) decimal places. Lorenzo dedicated his book, Geometria del compasso, to Napoleon Bonaparte. In this work, he proved that all Euclidean constructions can be made with compasses alone, so a straight edge in not needed. However, it was proved earlier in 1672 by the Danish mathematician Georg Mohr (1640-1697). He claimed that compasses are more accurate then those of a ruler.

1800. Karl Friedrich Gauss (1777-1855) was one of the greatest mathematicians of all time. Alexander von Humboldt (1769-1859), the famous traveler and amateur of the sciences, asked Pierre Simon de Laplace (1749-1827) who was the greatest mathematician in Germany, Laplace replied Johann Friedrich 'Pfaff' (1765-1825). 'But what about Gauss' the astonished von Humboldt asked, as he was backing Gauss for the position of director at 
the Göttingen observatory. 'Oh', said Laplace, 'Gauss is the greatest mathematician in the world' Gauss suggested to his teacher Pfaff to study the sequences $\left\{x_{n}\right\}$ and $\left\{y_{n}\right\}$ generated by the recurrence relations

$$
x_{n+1}=\frac{1}{2}\left(x_{n}+y_{n}\right), \quad y_{n+1}=\sqrt{x_{n+1} y_{n}}, \quad n \geq 0 .
$$

In his reply, Pfaff showed that for any positive numbers $x_{0}$ and $y_{0}$ these sequences converge monotonically to a common limit given by

$$
B\left(x_{0}, y_{0}\right)= \begin{cases}\left(y_{0}^{2}-x_{0}^{2}\right)^{1 / 2} / \cos ^{-1}\left(x_{0} / y_{0}\right), & 0 \leq x_{0}<y_{0}, \\ \left(x_{0}^{2}-y_{0}^{2}\right)^{1 / 2} / \cosh ^{-1}\left(x_{0} / y_{0}\right), & 0<y_{0}<x_{0} .\end{cases}
$$

Pfaff's letter was unpublished. In 1881, Carl Wilhelm Borchardt (1817-1880) work was published in which he rediscovered this result which now bears his name. For this, it suffices to note that:

1. $\left\{x_{n}\right\}$ and $\left\{y_{n}\right\}$ converge monotonically to the same limit.

2. The ratio $r_{n}=x_{n} / y_{n}$ satisfies $r_{n+1}^{2}=\left(1+r_{n}\right) / 2$.

3. If $x_{0}<y_{0}$, let $\theta=\cos ^{-1} r_{0}$. Then, $s_{n}=2^{n} \cos ^{-1} r_{n}=\theta$ and $c_{n}=4^{n}\left(x_{n}^{2}-y_{n}^{2}\right)=\left(x_{0}^{2}-y_{0}^{2}\right)$ are independent of $n$.

4. $\lim _{n \rightarrow \infty} y_{n}=\lim _{n \rightarrow \infty} \frac{2^{-n}\left|c_{n}\right|^{1 / 2}}{\sin ^{-1}\left(2^{-n}\left|c_{n}\right|^{1 / 2} / y_{n}\right)}=\lim _{n \rightarrow \infty} \frac{\left|c_{n}\right|^{1 / 2}}{s_{n}}=\frac{\left(y_{0}^{2}-x_{0}^{2}\right)^{1 / 2}}{\theta}$.

If $y_{0}<x_{0}$, we let $\theta=\cosh ^{-1} r_{0}$, and follow similarly.

Now we let $x_{n}=1 / a_{n}, y_{n}=1 / b_{n}$, then (25) and (26) take the form

$$
a_{n+1}=\frac{2 a_{n} b_{n}}{a_{n}+b_{n}}, \quad b_{n+1}=\sqrt{a_{n+1} b_{n}}, \quad n \geq 0
$$

and

$$
A\left(a_{0}, b_{0}\right)=\frac{1}{B\left(1 / a_{0}, 1 / b_{0}\right)}= \begin{cases}a_{0} b_{0}\left(a_{0}^{2}-b_{0}^{2}\right)^{-1 / 2} \cos ^{-1}\left(b_{0} / a_{0}\right), & a_{0}>b_{0} \geq 0, \\ a_{0} b_{0}\left(b_{0}^{2}-a_{0}^{2}\right)^{-1 / 2} \cosh ^{-1}\left(b_{0} / a_{0}\right), & b_{0}>a_{0}>0 .\end{cases}
$$

Clearly, the recurrence relations (2) are different from (27). In fact, (2) minimize the count of arithmetic operations. In particular, if we let $a_{0}=2 \sqrt{3}, b_{0}=3$, then (27) in view of (28) converges to $\pi$.

In what follows, we let the constant $c=4^{n}\left(x_{n}^{2}-y_{n}^{2}\right)=\left(x_{0}^{2}-y_{0}^{2}\right)$, then we can uncouple (25) and (27), respectively, to obtain

$$
x_{n+1}=\frac{x_{n}}{2}+\left(\left(\frac{x_{n}}{2}\right)^{2}-4^{-n-1} c\right)^{1 / 2}, \quad y_{n+1}^{2}=\frac{y_{n}^{2}}{2}\left(1+\left(1+4^{-n} c y_{n}^{-2}\right)^{1 / 2}\right)
$$

and

$$
a_{n+1}=\frac{2^{2 n+1}}{c a_{n}}\left(1-\left(1-4^{-n} c a_{n}^{2}\right)^{1 / 2}\right), \quad b_{n+1}^{2}=\frac{2^{2 n+1}}{c}\left(\left(1+4^{-n} c b_{n}^{2}\right)^{1 / 2}-1\right) .
$$

From (29) and (30), several known and new recurrence relations can be obtained.

Gauss also developed the Machin-type formula

$$
\frac{\pi}{4}=12 \tan ^{-1}\left(\frac{1}{18}\right)+8 \tan ^{-1}\left(\frac{1}{57}\right)-5 \tan ^{-1}\left(\frac{1}{239}\right) .
$$


He also estimated the value of $\pi$ by using lattice theory and considering a lattice inside a large circle, but he did not pursue it further.

1810. Sakabe Kohan (1759-1824) developed the series

$$
\frac{\pi}{4}=1-\frac{1}{5}-\frac{1 \cdot 4}{5 \cdot 7 \cdot 9}-\frac{(1 \cdot 3)(4 \cdot 6)}{5 \cdot 7 \cdot 9 \cdot 11 \cdot 13}-\frac{(1 \cdot 3 \cdot 5)(4 \cdot 6 \cdot 8)}{5 \cdot 7 \cdot 9 \cdot 11 \cdot 13 \cdot 15 \cdot 17}-\cdots .
$$

1818. Wada Yenzo Nei (known as Wada Yasushi, 1787-1840) developed over one hundred infinite series expressing directly or indirectly $\pi$. One of his series can be written as

$$
\pi=2 F\left(\frac{1}{2}, \frac{1}{2}, \frac{3}{2}, 1\right)=2\left(1+\frac{1^{2}}{3 !}+\frac{1^{2} \cdot 3^{2}}{5 !}+\frac{1^{2} \cdot 3^{2} \cdot 5^{2}}{7 !}+\cdots\right) .
$$

1825. Malacarne of Italy published a geometric construction in Géométrique (Paris), which leads to the value of $\pi$ less than 3 .

1828. C.G. Specht of Berlin published a geometric construction in Crelle's Journal, Volume 3, p.83, which leads to $\pi=13 \sqrt{146} / 50=3.1415919 \ldots$.

1832. Karl Heinrich Schellbach (1809-1890) began with the relation

$$
\frac{\pi i}{2}=\ln (i)=\ln \left(\frac{1+i}{1-i}\right)=\ln (1+i)-\ln (1-i)
$$

which is due to Giulio Carlo Fagnano dei Toschi (1682-1766), and used the logarithm expansion (7), to obtain

$$
\begin{aligned}
\frac{\pi i}{2} & =\left(i+\frac{1}{2}-\frac{1}{3} i-\frac{1}{4}+\frac{1}{5} i+\frac{1}{6}-\cdots\right)-\left(-i+\frac{1}{2}+\frac{1}{3} i-\frac{1}{4}-\frac{1}{5} i+\frac{1}{6}+\cdots\right) \\
& =2 i-\frac{2}{3} i+\frac{2}{5} i-\cdots,
\end{aligned}
$$

which immediately gives Nilakanthan series (5). He also considered the relation

$$
\begin{aligned}
\frac{\pi i}{2} & =\ln (i)=\ln \left(\frac{(2+i)(3+i)}{(2-i)(3-i)}\right) \\
& =\left\{\ln \left(1+\frac{1}{2} i\right)-\ln \left(1-\frac{1}{2} i\right)\right\}+\left\{\ln \left(1+\frac{1}{3} i\right)-\ln \left(1-\frac{1}{3} i\right)\right\}
\end{aligned}
$$

and used the expansion (7), to obtain, compare to (5), a fast converging expansion

$$
\frac{\pi}{4}=\left(\frac{1}{2}+\frac{1}{3}\right)-\frac{1}{3}\left(\frac{1}{2^{3}}+\frac{1}{3^{3}}\right)+\frac{1}{5}\left(\frac{1}{2^{5}}+\frac{1}{3^{5}}\right)-\cdots
$$

1833. William Baddeley in his work Mechanical quadrature of the circle, London Mechanics' Magazine, August, 1833 writes 'From a piece of carefully rolled sheet brass was cut out a circle 19/10 inches in diameter, and a square 1 7/10 inches in diameter. On weighing them, they were found to be exactly the same weight, which proves that, as each are of the same thickness, the surfaces must also be precisely similar. The rule, therefore, is that the square is to the circle as 17 to 19'. We believe for the square it must be the side (not the diameter). Then it follows that $\pi=1,156 / 361=3.202216 \ldots$. 
1836. Joseph LaComme at a time when he could neither read nor write being desirous to ascertain what quantity of stones would be required to prove a circular reservoir he had constructed, consulted a mathematics professor. He was told that it was impossible to determine the full amount, as no one had yet found the exact relation between the circumference of a circle and its diameter. The well-sinker thereupon, full of self-confidence in his powers, applied himself to the celebrated problem and discovered the solution, which lead to $\pi=25 / 8$ by mechanical process. He then taught himself to read and write, and managed to acquire some knowledge of arithmetic by which he verified his mechanical solution' Joseph was honored for his profound discovery with several medals of the first class, bestowed by Parisian societies.

1841. William Rutherford (1798-1871) was an English mathematician. He calculated $\pi$ to 208 places of which 152 were later found to be correct. For this, he employed Euler's formula

$$
\frac{\pi}{4}=4 \tan ^{-1}\left(\frac{1}{5}\right)-\tan ^{-1}\left(\frac{1}{70}\right)+\tan ^{-1}\left(\frac{1}{99}\right)
$$

and Madhava's series expansion (4).

1844. Johann Martin Zacharias Dase (1824-1861) was a calculating prodigy. At the age of 15, he gave exhibitions in Germany, Austria and England. His extraordinary calculating powers were timed by renowned mathematicians including Gauss. He multiplied 79,532,853 $\times 93,758,479$ in 54 seconds; two 20-digit numbers in 6 minutes; two 40-digit numbers in 40 minutes; and two 100-digit numbers in 8 hours 45 minutes. In 1840, he made acquaintance with Viennese mathematician L.K. Schulz von Strasznicky (1803-1852) who suggested him to apply his powers to scientific purposes. When he was 20, Strasznicky taught him the use of the formula

$$
\frac{\pi}{4}=\tan ^{-1}\left(\frac{1}{2}\right)+\tan ^{-1}\left(\frac{1}{5}\right)+\tan ^{-1}\left(\frac{1}{8}\right)
$$

and asked him to calculate $\pi$. In two months, he carried the approximation to 205 places of decimals, of which 200 are correct. He next calculated a 7-digit logarithm table of the first 1,005,000 numbers; he did this in his off-time from 1844 to 1847, when occupied by the Prussian survey. His next contribution of two years was the compilation of hyperbolic table in his spare time which was published by the Austrian Government in 1857. Next, he offered to make a table of integer factors of all numbers from 7,000,000 to 10,000,000; for this, on the recommendation of Gauss the Hamburg Academy of Sciences agreed to assist him financially, but Dase died shortly thereafter in Hamburg. He also had an uncanny sense of quantity. That is, he could just tell, without counting, how many sheep were in a field, or words in a sentence, and so forth, up to about 30.

1844. Hiromu Hasegawa (1810-1887) and his father Hiroshi Hasegawa (1782-1838) published many Wasan books. Hiromu developed the series

$$
\frac{\pi}{4}=1-\frac{1}{2 \cdot 3}-\frac{1}{5 \cdot 8}-\frac{1}{7 \cdot 16}-\frac{5}{9 \cdot 128}-\cdots .
$$

This series can be written as

$$
\pi=\frac{4}{3} F\left(\frac{1}{2}, \frac{3}{2}, \frac{5}{2}, 1\right) .
$$


1847. Thomas Clausen (1801-1885) wrote over 150 papers on pure mathematics, applied mathematics, astronomy and geophysics. He used the formula

$$
\frac{\pi}{4}=2 \tan ^{-1}\left(\frac{1}{3}\right)+\tan ^{-1}\left(\frac{1}{7}\right)
$$

to calculate $\pi$ to 250 decimal places, but only 248 are correct. In 1854, he factored the sixth Fermat number as $2^{64}+1=67,280,421,310,721 \times 274,177$. Clausen also gave a new method of factorising numbers.

1849. Jacob de Gelder (1765-1848) a mathematical ideologist proposed a geometric construction which gives $\pi$ correct to 6 decimal places. His method is based on the fact that $\pi=355 / 113=3+4^{2} /\left(7^{2}+8^{2}\right)$. Gelder's result was published in 1849 .

1851. Joseph Liouville (1809-1882) was a highly respected professor at the Collége de France in Paris, and the founder and for 39 years the editor of the Journal des Mathématiques Pures et Appliquées. His ingenious theory of fractional differentiation answered the long-standing question of what reasonable meaning can be assigned to the symbol $d^{n} y / d x^{n}$ when $n$ is not a positive integer. In 1844, Liouville showed that $e$ is not a root of any quadratic equation with integral coefficients. This led him to conjecture that $e$ is transcendental. In 1851, Liouville showed, by using continued fractions, that there are an infinite number of transcendental numbers, a result which had previously been suspected but had not been proved. He produced the first examples of real numbers that are provably transcendent. One of these is

$$
\sum_{n=1}^{\infty} \frac{1}{10^{n !}}=\frac{1}{10^{1}}+\frac{1}{10^{2}}+\frac{1}{10^{6}}+\cdots=0.11000100 \ldots
$$

His methods led to extensive further research.

1853. Lehmann correctly calculated 261 decimal places of $\pi$. For this, he used Euler's formula

$$
\frac{\pi}{4}=\tan ^{-1}\left(\frac{1}{2}\right)+\tan ^{-1}\left(\frac{1}{3}\right) .
$$

1853. Rutherford obtained 440 correct decimal places.

1853. William Shanks (1812-1882) was a British amateur mathematician. He used Machin formula (18) to calculate $\pi$ to 607 decimal places. He was assisted by Rutherford in checking first 440 digits.

1853-1855. Richter in 1853 published 333 digits (330 correct), and in 1855 (after his death in 1854) 500 decimal places.

1860. James Smith published the value of $\pi$ as $31 / 8$ and argued that it is exact and correct. He attempted to bring it before the British Association for the Advancement of Science. Interestingly, even De Morgan and Hamilton could not convince him for his mistake.

1861. Philip H. Vanderweyde published an essay discussing the subject $\pi$. He also used several constructions, resulting $\pi=3.1415926535 \ldots$.

1862. Lawrence Sluter Benson published about 20 pamphlets on the area of the circle, three volumes on philosophic essays, and one on geometry The Elements of Euclid and Legendre. He demonstrated that the area of the circle is equal to $3 R^{2}$, or the 
arithmetical square between the inscribed and circumscribed squares. According to him $\sqrt{12}=3.4641016 \ldots$ is the ratio between the diameter of a circle and the perimeter of its equivalent square. The ratio between the diameter and circumference, he believed, is not a function of the area of the circle. He accepted the value of $\pi=3.141592 \ldots$.

1863. S.M. Drach proved that the circumference of a circle can be obtained as follows: From thrice diameter, deduct $8 / 1,000$ and $7 / 1,000,000$ of a diameter, and add $5 \%$ to the result, i.e.,

$$
2 \pi=6-\frac{16}{1,000}-\frac{14}{1,000,000}+\frac{5}{100}\left(6-\frac{16}{1,000}-\frac{14}{1,000,000}\right),
$$

which gives $\pi=3.14159265$.

1868. Cyrus Pitt Grosvenor (1792-1879) was an American anti-slavery Baptist minister. In his retirement, he worked on the problem of squaring the circle. He described his method in a pamphlet titled The circle squared, New York: Square the diameter of the circle; multiply the square by 2 ; extract the square root of the product; from the root subtract the diameter of the circle; square the remainder; multiply this square by four fifths; subtract the square from the diameter of the circle, i.e.,

$$
\frac{\pi D^{2}}{4}=D^{2}-\frac{5}{4}\left(\sqrt{2 D^{2}}-D\right)^{2}=D^{2}\left[1-\frac{5}{4}(\sqrt{2}-1)^{2}\right]=D^{2}(0.785533906 \ldots)
$$

which gives $\pi=3.142135 \ldots$.

1872. Augustus De Morgan (1806-1871) was born in Madura (India), but his family moved to England when he was seven months old. He lost the sight of his right eye shortly after birth. He was an extremely prolific writer. He wrote more than 1,000 articles for more than 15 periodicals. De Morgan also wrote textbooks on many subjects, including logic, probability, calculus and algebra. In 1866, he was a co-founder of the London Mathematical Society and became its first President. His book A Budget of Paradoxes of 512 pages, which was edited and published by his wife in 1872, is an entertaining text. This book contains the names of 75 writers on $\pi$. In this work, De Morgan reviewed the works of 42 of these writers, bringing the subject down to 1870 . He once remarked that it is easier to square the circle then to get round a mathematician. He was the first to point out that in the decimal expansion of $\pi$ one should expect each of the 10 digits appear uniformly, i.e., roughly one out of ten digits should be a 4 , etc.

1872. Asaph Hall (1829-1907) was an astronomer. He published the results of an experiment in random sampling that Hall had convinced his friend, Captain O.C. Fox, to perform when Fox was recovering from a wound received at the Second Battle of Bull Run. The experiment was based on Buffon's needle problem. After throwing his needles eleven hundred times, Fox was able to derive $\pi \simeq 3.14$. This work is considered as a very early documentation use of random sampling (which Nicholas Constantine Metropolis (1915-1999) named as the Monte Carlo method during the Manhattan Project of World War II).

1873. Charles Hermite (1822-1901) in 1870 was appointed to a professorship at the Sorbonne, where he trained a whole generation of well-known French mathematicians. He was strongly attracted to number theory and analysis, and his favorite subject was elliptic functions, where these two fields touch in many remarkable ways. His proof of the transcendence of $e$ was high point in his career. 
1873-1874. William Shanks again used Machin formula (18) to calculate $\pi$ to $707 \mathrm{dec}-$ imal places (published in the Proceedings of the Royal Society, London), but only 527 decimal places are correct. For this, he used mechanical desk calculator and worked for almost 15 years. For a long time, this remained the most fabulous piece of calculation ever performed. In the Palais de la Découverte (a science museum in Paris), there is a circular room known as the 'pi room. On its wall are inscribed these 707 digits of $\pi$. The digits are large wooden characters attached to the dome-like ceiling. Shanks also calculated $e$ and the Euler-Mascheroni constant $\gamma$ to many decimal places. He published a table of primes up to 60,000 and found the natural logarithms of 2, 3, 5 and 10 to 137 places.

1874. Tseng Chi-Hung (died in 1877) finds 100 digits of $\pi$ in a month. For this, he used the formula (32).

1874. John A. Parker in his book The Quadrature of the Circle. Containing Demonstrations of the Errors of Geometers in Finding Approximations in Use published by John Wiley \& Sons, New York claims that $\pi=20,612 / 6,561$ exactly. He exclaims, 'all the serial and algebraic formula in the world, or even geometrical demonstration, if it be subjected to any error whatever, cannot overthrow the ratio of circumference to diameter which I have established. He praises Metius (lived in the sixteenth century) for using the ratio 355/113. His book also contains practical questions on the quadrature applied to the astronomical circles.

1876. Alick Carrick proposed in his book, The Secret of the Circle, its Area Ascertained, the value of $\pi$ as $31 / 7$.

1879. Pliny Earle Chase (1820-1886) was a scientist, mathematician, and educator who mainly contributed to the fields of astronomy, electromagnetism and cryptography. In his pamphlet, Approximate Quadrature of the Circle, he used a geometric construction to obtain $\pi=3.14158499 \ldots$.

1882. Carl Louis Ferdinand von Lindemann (1852-1939) worked on non-Euclidean geometry. He followed the method of Hermite to show that $\pi$ is also transcendental. His result showed at last that the age-old problem of squaring the circle by a ruler-and-compass construction is impossible. Lindemann's paper runs to 13 pages of tough mathematics. Karl Wilhelm Weierstrass (1815-1897), the apostle of mathematical rigor, simplified the proof of Lindemann's theorem somewhat in 1885, and it was further simplified in later years by renowned mathematicians (Stieltjes, Hurwitz, Hilbert, and others). The interested reader is referred to the comparatively easy version given by Hobson. Nonetheless, there are still some amateur mathematicians who do not understand the significance of this result, and futilely look for techniques to square the circle. Next, Lindemann spent several years to provide the proof of Fermat's Last Theorem, which is unfortunately wrong. He also worked on projective geometry, Abelian functions and developed a method of solving equations of any degree using transcendental functions.

1888. Sylvester Clark Gould (1840-1909) was the editor of Notes and Queries, Manchester, New Hampshire. He compiled the bibliography entitled What is the Value of Pi. It contains 100 titles and gives the result of 63 authors. In this work the diagram 16 claims that $\pi=33,949 / 27,889$ exactly.

1892. A writer announced in the New York Tribune the rediscovery of a long-lost secret that gives 3.2 as the exact value of $\pi$. This announcement caused considerable discussion, and even near the beginning of the twentieth century 3.2 had its advocates as against the value $22 / 7$. 
1896. Fredrik Carl Mülertz Störmer (1874-1957) was a mathematician and physicist, known for his work in number theory. He gave the following Machin-like formulas for calculating $\pi$

$$
\frac{\pi}{4}=44 \tan ^{-1}\left(\frac{1}{57}\right)+7 \tan ^{-1}\left(\frac{1}{239}\right)-12 \tan ^{-1}\left(\frac{1}{682}\right)+24 \tan ^{-1}\left(\frac{1}{12,943}\right)
$$

and

$$
\frac{\pi}{4}=6 \tan ^{-1}\left(\frac{1}{8}\right)+2 \tan ^{-1}\left(\frac{1}{57}\right)+\tan ^{-1}\left(\frac{1}{239}\right) .
$$

1897. In the State of Indiana, the House of Representatives unanimously passed the Bill No. 246 (known as the ' $\pi$ bill') introducing a new mathematical truth 'Be it enacted by the General Assembly of the State of Indiana: It has been found that a circular area is to the square on a line equal to the quadrant of the circumference, as the area of an equilateral rectangle is to the square on one side...' $(\pi=3.2)$. The author of the bill was a physician, Edwin J. Goodman (1825-1902), M.D., of Solitude, Posey County, Indiana, and it was introduced in the Indiana House on 18 January 1897, by Mr. Taylor I. Record, representative from Posey County. Edwin offered this contribution as a free gift for the sole use of the State of Indiana (the others would evidently have to pay royalties). Copies of the bill are preserved in the Archives Division of the Indiana State Library. The bill was sent to the Senate for approval. Fortunately, during the House's debate on the bill, Purdue University Mathematics Professor Clarence Abiathar Waldo (1852-1926) was present. When Professor Waldo informed the Indiana Senate of the 'merits' of the bill, the Senate, after some ridicule at the expense of their colleagues, indefinitely postponed voting on the bill and let it die.

1900. H.S. Uhler used Machin's formula (18) to compute $\pi$ to 282 decimal places.

1901. Mario Lazzarini an Italian mathematician performed the Buffon's needle experiment. Tossing a needle 3,408 times, he obtained the well-known estimate 355/113 for $\pi$. Although it is an impressive observation, but suspiciously good. In fact, statisticians Sir Maurice George Kendall (1907-1983) and Patrick Alfred Pierce Moran (1917-1988) FRS have commented that one can do better to cut out a large circle and use a tape to measure to find its circumference and diameter. On the same theme of phoney experiments, Gridgeman, in 1960, pours scorn on Lazzerini and others, created some amusement by using a needle of carefully chosen length $k=0.7857$, throwing it twice, and hitting a line once. His estimate for $\pi$ was thus given by $2 \times 0.7857 / \pi=1 / 2$ from which he got the highly creditable value of $\pi=3.1428$. Of course, he was not being serious.

1902. Duarte used Machin's formula (18) to compute $\pi$ to 200 decimal places.

1906. Various mnemonic devices have been given for remembering the decimal digits of $\pi$. The most common type of mnemonic is the word-length mnemonic in which the number of letters in each word corresponds to a digit, for example, How I wish I could calculate pi (by C. Heckman), May I have a large container of coffee (by Martin Gardner), and How I want a drink, alcoholic of course, after the heavy lectures involving quantum mechanics (by Sir James Jeans), respectively, give $\pi$ to seven, eight, and fifteen decimal places. Adam C. Orr in Literary Digest, vol. 32 (1906), p.84 published the following poem which gives $\pi$ to 30 decimal places: 
Now I, even I, would celebrate

In rhymes inapt, the great

Immortal Syracusan, rivaled nevermore

Who in his wondrous lore,

Passed on before

Left men his guidance,

How to circles mensurate.

Several other such poems not only in English, but almost in every language including Albanian, Bulgarian, Czech, Dutch, French, German, Italian, Latin, Polish, Portuguese, Romanian, Spanish and Swedish are known. However, there is a problem with this type of mnemonic, namely, how to represent the digit zero. Fortunately, a zero does not occur in $\pi$ until the thirty-second place. Several people have come up with ingenious methods of overcoming this, most commonly using a ten-letter word to represent zero. In other cases, a certain piece of punctuation is used to indicate a naught. Michael Keith (with such similar understanding) in his work Circle digits: a self-referential story, Mathematical Intelligencer, vol. 8 (1986), 56-57, wrote an interesting story which gives first 402 decimals of $\pi$.

1913. Ernest William Hobson (1856-1933) was Sadleirian Professor at the University of Cambridge from 1910 to 1931 . His 1907 work on real analysis was very influential in England. In his book, Squaring the circle: A History of the Problem, he used a geometrical construction to obtain $\pi=3.14164079 \ldots$.

1914. Srinivasa Ramanujan (1887-1920) was a famous mathematical prodigy. He collaborated with Hardy for five years, proving significant theorems about the number of partitions of integers. Ramanujan also made important contributions to number theory and also worked on continued fractions, infinite series and elliptic functions. In 1918, he became the youngest Fellow of the Royal Society. According to Hardy, 'the limitations of Ramanujan's knowledge were as startling as its profundity'. Here was a man who could workout modular equations and theorems of complex multiplication, to orders unheard of, whose mastery of continued fractions was, beyond that of any mathematician in the world, who had found for himself the functional equation of the zeta-function, and the dominant terms of the many of the most famous problems in the analytic theory of numbers; and he had never heard of a doubly periodic function or of Cauchy's theorem, and had indeed but the vaguest idea of what a function of a complex variable was. Ramanujan considered mathematics and religion to be linked. He said, 'an equation for me has no meaning unless it expresses a thought of God.' He was endowed with an astounding memory and remembered the idiosyncrasies of the first 10,000 integers to such an extent that each number became like a personal friend to him. Once Hardy went to see Ramanujan when he was in a nursing home and remarked that he had traveled in a taxi with a rather dull number, viz 1,729, Ramanujan exclaimed, 'No, Hardy, 1,729 is a very interesting number. It is the smallest number that can be expressed as the sum of two cubes viz $1,729=1^{3}+12^{3}=9^{3}+10^{3}$, and the next such number is very large'. His life can be summed up in his own words, 'I really love my subject.' His 1914 paper on 'Modulus functions and approximation to $\pi$ ' contains several new innovative empirical formulas and geometrical constructions for approximating $\pi$. One of the remarkable formulas for its elegance and inherent mathematical depth 


$$
\frac{1}{\pi}=\frac{\sqrt{8}}{9,801} \sum_{m=0}^{\infty} \frac{(4 m) !}{(m !)^{4}} \frac{(1,103+26,390 m)}{396^{4 m}} .
$$

It has been used to compute $\pi$ to a level of accuracy, never attained earlier. Each additional term of the series adds roughly 8 digits. He also developed the series

$$
\frac{1}{\pi}=\sum_{m=0}^{\infty}\left(\begin{array}{c}
2 m \\
m
\end{array}\right)^{3} \frac{42 m+5}{2^{12 m+4}} \quad \text { and } \quad \frac{2}{\pi}=\sum_{m=0}^{\infty} \frac{(-1)^{m}(4 m+1)[(2 m-1) ! !]^{3}}{((2 k) ! !)^{3}}
$$

The first series has the property that it can be used to compute the second block of $k$ (binary) digits in the decimal expansion of $\pi$ without calculating the first $k$ digits. The following mysterious approximation which approximates $\pi$ to 18 correct decimal places is also due to Ramanujan

$$
\pi \simeq \frac{12}{\sqrt{190}} \ln ((2 \sqrt{2}+\sqrt{10})(3+\sqrt{10}))
$$

1914. T.M.P. Hughes in his work $A$ triangle that gives the area and circumference of any circle, and the diameter of a circle equal in area to any given square, Nature 93, 110, doi:10.1038/093110a0 uses a geometric construction to obtain $\pi=3.14159292035 \ldots$.

1928. In March 1928, the University of Minnesota was notified that Gottfried Lenzer (a native of Germany who lived in St. Paul for many years) had bequeathed to the university a series of 60 drawings from 1911-1927 and explanatory notes concerning the three classical problems of antiquity. He used a geometrical construction for squaring the circle to obtain $\pi=3.1378 \ldots$.

1934. Alexander Osipovich Gelfond (1906-1968) was a Soviet mathematician. He proved that $e^{\pi}$ (Gelfond's constant) is transcendental, but nothing yet is known about the nature of any of the numbers $\pi+e, \pi e$ or $\pi^{e}$.

1934. Helen Abbot Merrill (1864-1949) earned her Ph.D. from Yale in 1903 on the thesis On Solutions of Differential Equations which Possess an Oscillation Theorem. She served as an associate editor of The American Mathematical Monthly during 1916-1919, and was a vice-president from 1920 to 1921 of the Mathematical Association of America. Her book Mathematical Excursions: Side Trips Along Paths not Generally Traveled in Elementary Courses in Mathematics, Bruce Humphries, Inc., Boston, 1934 was a text for the general public. In this book, a geometric construction is given (perhaps by an earlier author) which leads to $\pi=3.141591953 \ldots$.

1934. Edmund Georg Hermann (Yehezkel) Landau (1877-1938) was a child prodigy. In 1903, he gave a simpler proof of the prime number theorem. His masterpiece of 1909 was a treatise Handbuch der Lehre von der Verteilung der Primzahlen a two volume work giving the first systematic presentation of analytic number theory. Landau wrote over 250 papers on number theory, which had a major influence on the development of the subject. Despite his outstanding talents as both a teacher and researcher, Landau annoyed many of his colleagues at Göttingen. He started criticizing privately, and often publicly, their results. Landau in his work defined $\pi / 2$ as the value of $x$ between 1 and 2 for which $\cos x$ vanishes. One cannot believe this definition was used, at least as an excuse, for a racial attack on 
Landau. This unleashed an academic dispute which was to end in Landau's dismissal from his chair at Göttingen. Ludwig Georg Elias Moses Bieberbach (1886-1982) famous for his conjecture, explained the reasons for Landau's dismissal: 'Thus the valiant rejection by the Göttingen student body which a great mathematician, Edmund Landau, has experienced is due in the final analysis to the fact that the un-German style of this man in his research and teaching is unbearable to German feelings. A people who have perceived how members of another race are working to impose ideas foreign to its own must refuse teachers of an alien culture.' Hardy replied immediately to Bieberbach about the consequences of this un-German definition of $\pi$ : 'There are many of us, many Englishmen and many Germans, who said things during the War which we scarcely meant and are sorry to remember now. Anxiety for one's own position, dread of falling behind the rising torrent of folly, determination at all cost not to be outdone, may be natural if not particularly heroic excuses. Professor Bieberbach's reputation excludes such explanations of his utterances, and I find myself driven to the more uncharitable conclusion that he really believes them true.'

1934. A Cleveland businessman Carl Theodore Heisel published a book Mathematical and Geometrical Demonstrations in which he announced the grand discovery that $\pi$ was exactly equal to 256/81, a value that the Egyptians had used some 4,000 years ago. Substituting this value for calculations of areas and circumferences of circles with diameters $1,2, \ldots$ up to 9 , he obtained numbers which showed consistency of circumference and area, 'thereby furnishing incontrovertible proof of the exact truth' of his ratio. He also rejected decimal fractions as inexact (whereas ratios of integers as exact and scientific), and extracted roots of negative numbers thus: $\sqrt{-a}=\sqrt{a}-, \sqrt{a-2}=-a$. He published this book on his own expense and distributed to colleges and public libraries throughout the United States without charge.

1934. Miff Butler claimed discovery of a new relationship between $\pi$ and $e$. He stated his work to be the first basic mathematical principle ever developed in USA. He convinced his congressman to read it into the Congressional Record on 5 June 1940.

1940. H.S. Uhler used Machin's formula (18) to compute $\pi$ to 333 decimal places.

1945-1947. D.F. Ferguson of England used the formula

$$
\frac{\pi}{4}=3 \tan ^{-1}\left(\frac{1}{4}\right)+\tan ^{-1}\left(\frac{1}{20}\right)+\tan ^{-1}\left(\frac{1}{1,985}\right)
$$

to find that his value disagreed with that of William Shanks in the 528th place. In 1946, he approximated $\pi$ to 620 decimal places, and in January 1947 to 710 decimal places. In the same month William Shanks used Machin's formula (18) to compute 808-place value of $\pi$, but Ferguson soon found an error in the 723rd place. For all the calculations, he used desk calculator.

1947. Ivan Morton Niven (1915-1999) gave an elementary proof that $\pi$ is irrational.

1949. Ferguson and John William Wrench, Jr. (1911-2009) using a desk calculator, computed 1,120 decimal digits of $\pi$. This record was broken only by the electronic computers.

September 1949. John Wrench and L.R. Smith (also attributed to George Reitwiesner et al.) were the first to use an electronic computer Electronic Numerical Integrator and Computer (ENIAC) at the Army Ballistic Research Laboratories in Aberdeen, Maryland, to calculate $\pi$ to 2,037 decimal places. For this, they programed Machin's formula (18). It took 70 hours, a pitifully long time by today's standards. In this project, John Louis von Neumann (1903-1957), one of the most versatile and smartest mathematicians of the 
twentieth century, also took part. In 1965, The ENIAC became obsolete, and it was dismembered and moved to the Smithsonian Institution as a museum piece.

1951. Konrad Knopp gave the following two expansions of $\pi$ :

$$
\frac{\pi}{4}=\sum_{k=1}^{\infty} \tan ^{-1}\left(\frac{1}{k^{2}+k+1}\right) \text { and } \frac{\pi^{2}}{16}=\sum_{k=0}^{\infty} \frac{(-1)^{k}}{k+1}\left(1+\frac{1}{3}+\cdots+\frac{1}{2 k+1}\right) .
$$

1953. Kurt Mahler (1903-1988) showed that $\pi$ is not a Liouville number: A real number $x$ is called a Liouville number if for every positive integer $n$, there exist integers $p$ and $q$ with $q>1$ and such that

$$
0<\left|x-\frac{p}{q}\right|<\frac{1}{q^{n}} .
$$

A Liouville number can thus be approximated 'quite closely' by a sequence of rational numbers. In 1844, Liouville showed that all Liouville numbers are transcendental.

1954. S.C. Nicholson and J. Jeenel programmed NORC (Naval Ordnance Research Calculator) at Dahlgren, Virginia to compute $\pi$ to 3,092 decimals. For this, they used Machin's formula (18). The run took only 13 minutes.

1956. John Gurland established that for all positive integers $n$,

$$
\frac{4 n+3}{(2 n+1)^{2}}\left(\frac{(2 n) ! !}{(2 n-1) ! !}\right)^{2}<\pi<\frac{4}{4 n+1}\left(\frac{(2 n) ! !}{(2 n-1) ! !}\right)^{2}
$$

March 1957. G.E. Felton used the Ferranti Pegasus computer to find 10,021 decimal places of $\pi$ in 33 hours. The program was based on Klingenstierna's formula

$$
\pi=32 \arctan \left(\frac{1}{10}\right)-4 \arctan \left(\frac{1}{239}\right)-16 \arctan \left(\frac{1}{515}\right) \text {. }
$$

However, a subsequent check revealed that a machine error had occurred, so that 'only' 7,480 decimal places were correct. The run was therefore repeated in May 1958, but the correction was not published.

January 1958. Francois Genuys programmed an IBM 704 at the Paris Data Processing Center. He used Machin's type formula (18). It yielded 10,000 decimal places of $\pi$ in 1 hour and 40 minutes.

July 1959. Genuys programmed an IBM 704 at the Commissariat á l'Energie Atomique in Paris to compute $\pi$ to 16,167 decimal places. He used Machin's type formula (18). It took 4 hours and 30 minutes.

July 1961. Daniel Shanks (1917-1996) and William Shanks used Störmer's formula (34) on an IBM 7090 (at the IBM Data Processing Center, New York) to compute $\pi$ to 100,265 digits, of which the first 100,000 digits were published by photographically reproducing the print-out with 5,000 digits per page. The time required for this computation was 8 hours and 43 minutes. They also checked the calculations by using Gauss' formula (31), which required 4 hours and 22 minutes.

1961. Machin's formula (18) was also the basis of a program run on an IBM 7090 at the London Data Center in July 1961, which resulted in 20,000 decimal places and required only 39 minutes running time. 
February 1966. Jean Guilloud and J. Filliatre used an IBM 7030 at the Commissariat á l'Energie Atomique in Paris to obtain an approximation of $\pi$ extending to 250,000 decimal places on a STRETCH computer. For this, they used Störmer's and Gauss' formulas (34) and (31). It took almost 28 hours.

February 1967. Guilloud and M. Dichampt used CDC (Control Data Corporation) 6600 in Paris to approximate $\pi$ to 500,000 decimal places. For this, they used Störmer's and Gauss' formulas (34) and (31). The computer that churned out half a million digits needed only 26 hours and 40 minutes (plus 1 hour and 30 minutes to convert that final result from binary to decimal notation).

1968. In the Putnam Competition, the first problem was

$$
\pi=\frac{22}{7}-\int_{0}^{1} \frac{x^{4}(1-x)^{4}}{1+x^{2}} d x
$$

This integral was known to Mahler in the mid-1960s, and has later appeared in several exams. It is also discussed by Borwein, Bailey, and Girgensohn in their book on p.3.

1971. K.Y. Choong, D.E. Daykin and C.R. Rathbone used 100,000 digits of Daniel Shanks and William Shanks (1961) to generate the first 21,230 partial quotients of the continued fraction expansion of $\pi$.

1974. Ralph William Gosper, Jr. (born 1943), known as Bill Gosper, is a mathematician and programmer. He is best known for the symbolic computation, continued fraction representations of real numbers, Gosper's algorithm, and Gosper curve. He used a refinement of Euler transform on (5) to obtain the series

$$
\begin{aligned}
\pi= & +\frac{1}{60} 8+\frac{1}{60} \frac{2 \cdot 3}{7 \cdot 8 \cdot 3} 13+\frac{1}{60} \frac{2 \cdot 3}{7 \cdot 8 \cdot 3} \frac{3 \cdot 5}{10 \cdot 11 \cdot 3} 18 \\
& +\frac{1}{60} \frac{2 \cdot 3}{7 \cdot 8 \cdot 3} \frac{3 \cdot 5}{10 \cdot 11 \cdot 3} \frac{4 \cdot 7}{13 \cdot 14 \cdot 3} 23+\cdots
\end{aligned}
$$

1974. Guilloud with Martine Bouyer (Paris) used formulas (34) and (31) on a CDC 7600 to compute $\pi$ to $1,000,250$ digits. The run time required for this computation was 23 hours and 18 minutes, of which 1 hour 7 minutes was used to convert the final result from binary to decimal. Results of statistical tests, which generally support the conjecture that $\pi$ is simply normal (in 1909, Félix Édouard Justin Émil Borel (1871-1956) defined: A real number $a$ is simply normal in base $b$ if in its representation in base $b$ all digits occur, in an asymptotic sense, equally often) were also performed.

1974. Louis Comtet developed the following Euler's type expansion of $\pi$ :

$$
\frac{\pi^{4}}{90}=\frac{36}{17} \sum_{m=1}^{\infty} \frac{1}{m^{4}\left(\begin{array}{c}
2 m \\
m
\end{array}\right)} .
$$

1976. Richard Brent and Eugene Salamin independently discovered an algorithm which is based on an arithmetic-geometric mean and modifies slightly Gauss-Legendre algorithm. Set $a_{0}=1, b_{0}=1 / \sqrt{2}$ and $s_{0}=1 / 2$. For $k=1,2,3, \ldots$ compute

$$
\begin{aligned}
& a_{k}=\frac{a_{k-1}+b_{k-1}}{2}, \\
& b_{k}=\sqrt{a_{k-1} b_{k-1}},
\end{aligned}
$$




$$
\begin{aligned}
& c_{k}=a_{k}^{2}-b_{k}^{2}, \\
& s_{k}=s_{k-1}-2^{k} c_{k}, \\
& p_{k}=\frac{2 a_{k}^{2}}{s_{k}} .
\end{aligned}
$$

Then $p_{k}$ converges quadratically to $\pi$, i.e., each iteration doubles the number of accurate digits. In fact, successive iterations must produce 1, 4, 9, 20, 42, 85, 173, 347 and 697 correct digits of $\pi$. The twenty-fifth iteration must produce 45 million correct decimal digits of $\pi$.

1981. Kazunori Miyoshi and Kazuhika Nakayama of the University of Tsukuba, Japan calculated $\pi$ to 2,000,038 significant figures in 137.30 hours on a FACOM M-200 computer. They used Klingenstierna's formula (37) and checked their result with Machin's formula (18).

1981. Guilloud computed $2,000,050$ decimal digits of $\pi$.

1981. Rajan Srinivasan Mahadevan (born 1957) recited from memory the first 31,811 digits of $\pi$. This secured him a place in the 1984 Guinness Book of World Records, and he has been featured on Larry King Live and Reader's Digest.

1982. Kikuo Takano (1927-2006) was a Japanese poet and mathematician. He developed the following Machin-like formula for calculating $\pi$ :

$$
\frac{\pi}{4}=12 \tan ^{-1}\left(\frac{1}{49}\right)+32 \tan ^{-1}\left(\frac{1}{57}\right)-5 \tan ^{-1}\left(\frac{1}{239}\right)+12 \tan ^{-1}\left(\frac{1}{110,443}\right) .
$$

1982. Yoshiaki Tamura on MELCOM 900 II computed 2,097,144 decimal places of $\pi$. For this, he used the Salamin-Brent algorithm (38).

1982. Yoshiaki Tamura and Yasumasa Kanada (born 1948, life-long 'pi digit-hunter', set the record 11 of the past 21 times) on HITAC M-280H computed 4,194,288 decimal places of $\pi$. For this, they used the Salamin-Brent algorithm (38).

1982. Yoshiaki Tamura and Yasumasa Kanada on HITAC M-280H computed 8,388,576 decimal places of $\pi$. For this, they used the Salamin-Brent algorithm (38).

October 1983. Yasumasa Kanada, Yoshiaki Tamura, Sayaka Yoshino and Yasunori Ushiro on HITAC S-810/20 computed 10,013,395 decimal places of $\pi$. For this, they used the Salamin-Brent algorithm (38). In this work to gather evidence that $\pi$ is simply normal, they also performed statistical analysis. It showed expected behavior. In the first ten million digits, the frequencies for each ten digits are 999,440;999,333; 1,000,306; 999,$964 ; 1,001,093 ; 1,000,466 ; 999,337 ; 1,000,207 ; 999,814$; and 1,000,040. Further, the rate at which the relative frequencies approach $1 / 10$ agrees with theory. As an example, for the digit 7 relative frequencies in the first $10^{i}, i=0,1,2,3,4,5,6,7$ digits are $0,0.08,0.095,0.097,0.10025,0.0998,0.1000207$, which seem to be approaching $1 / 10$ at rate predicted by the probability theory for random digits, i.e., a speed approximately proportional to $1 / \sqrt{n}$. But this is far from a formal proof of simple normalcy perhaps for a proof the current mathematics is not sufficiently developed. In spite of the fact that the digits of $\pi$ pass statistical tests for randomness, $\pi$ contains some sequences of digits that, to some, may appear non-random, such as Feynman point, which is a sequence of six consecutive 9s that begins at the 762nd decimal place. A number is said to be normal if all blocks of digits of the same length occur with equal frequency. Mathematicians expect $\pi$ to be normal, so that every pattern possible eventually will occur in the digits of $\pi$. 
1983. Yasumasa Kanada, Sayaka Yoshino and Yoshiaki Tamura on HITAC M-280H computed 16,777,206 decimal places of $\pi$. For this, they used the Salamin-Brent algorithm (38).

1984. Jonathan Borwein and Peter Borwein gave the following algorithm. Set $x_{0}=\sqrt{2}$, $y_{0}=0$ and $\alpha_{0}=2+\sqrt{2}$. Iterate

$$
\begin{aligned}
& x_{k+1}=\left(\sqrt{x_{k}}+1 / \sqrt{x_{k}}\right) / 2, \\
& y_{k+1}=\sqrt{x_{k}}\left(\frac{1+y_{k}}{y_{k}+x_{k}}\right), \\
& \alpha_{k+1}=\alpha_{k} y_{k+1}\left(\frac{1+x_{k+1}}{1+y_{k+1}}\right) .
\end{aligned}
$$

Then $\alpha_{k}$ converges to $\pi$ quartically. The algorithm is not self-correcting; each iteration must be performed with the desired number of correct digits of $\pi$.

1984. Morris Newman and Daniel Shanks proved the following: Set

$$
\begin{aligned}
& a=\frac{1,071}{2}+92 \sqrt{34}+\frac{3}{2} \sqrt{255,349+43,792 \sqrt{34}} \\
& b=\frac{1,533}{2}+133 \sqrt{34}+\frac{1}{2} \sqrt{4,817,509+826,196 \sqrt{34}} \\
& c=429+304 \sqrt{2}+2 \sqrt{92,218+65,208 \sqrt{2}}, \\
& d=\frac{627}{2}+221 \sqrt{2}+\frac{1}{2} \sqrt{783,853+554,268 \sqrt{2}}
\end{aligned}
$$

then

$$
\left|\pi-\frac{6}{\sqrt{3,502}} \ln (2 a b c d)\right|<7.4 \times 10^{-82} .
$$

1985. Gosper used Symbolics 3,670 and Ramanujan's formula (35) to compute $\pi$ to 17,526,200 decimal digits.

1985. Jonathan Borwein and Peter Borwein gave the following algorithm. Set $a_{0}=6-$ $4 \sqrt{2}$ and $y_{0}=\sqrt{2}-1$. Iterate

$$
\begin{aligned}
& y_{k+1}=\frac{1-\left(1-y_{k}^{4}\right)^{1 / 4}}{1+\left(1-y_{k}^{4}\right)^{1 / 4}}, \\
& a_{k+1}=a_{k}\left(1+y_{k+1}\right)^{4}-2^{2 k+3} y_{k+1}\left(1+y_{k+1}+y_{k+1}^{2}\right) .
\end{aligned}
$$

Then $a_{k}$ converges quartically to $1 / \pi$, i.e., each iteration approximately quadruples the number of correct digits.

1985. The following is not an identity, but is correct to over 42 billion digits

$$
\left(\frac{1}{10^{5}} \sum_{n=-\infty}^{\infty} e^{-n^{2} / 10^{10}}\right)^{2} \simeq \pi
$$

1985. Carl Sagan in his novel deals with the theme of contact between humanity and a more technologically advanced, extraterrestrial life form. He suggests that the creator of the universe buried a message deep within the digits of $\pi$. 
January 1986. David H. Bailey used Borweins' algorithms (40) and (41) on CRAY-2 to compute 29,360,111 decimal places of $\pi$.

September 1986. Yasumasa Kanada and Yoshiaki Tamura on HITAC S-810/20 computes 33,554,414 decimal places of $\pi$. For this, they used algorithms (38) and (41).

October 1986. Yasumasa Kanada and Yoshiaki Tamura on HITAC S-810/20 computed $67,108,839$ decimal places of $\pi$. For this, they used algorithm (38).

January 1987. Yasumasa Kanada, Yoshiaki Tamura, Yoshinobu Kubo and others on NEC SX-2 computed 134,214,700 decimal places of $\pi$. For this they used algorithms (38) and (41).

1987. Jonathan Borwein and Peter Borwein gave the following algorithm. Set $x_{0}=2^{1 / 2}$, $y_{1}=2^{1 / 4}$ and $p_{0}=2+2^{1 / 2}$. Iterate

$$
\begin{aligned}
& x_{k}=\frac{1}{2}\left(x_{k-1}^{1 / 2}+x_{k-1}^{-1 / 2}\right), \\
& y_{k}=\frac{y_{k-1} x_{k-1}^{1 / 2}+x_{k-1}^{-1 / 2}}{y_{k-1}+1}, \\
& p_{k}=p_{k-1} \frac{x_{k}+1}{y_{k}+1} .
\end{aligned}
$$

Then $p_{k}$ decreases monotonically to $\pi$ and $\left|p_{k}-\pi\right| \leq 10^{-2^{k+1}}$ for $k \geq 4$.

1987. Hideaki Tomoyori (born 1932) recited $\pi$ from memory to 40,000 places taking 17 hours 21 minutes, including breaks totaling 4 hours 15 minutes, at Tsukuba University Club House.

January 1988. Yasumasa Kanada on HITAC S-820/80 computed 201,326,551 decimal places of $\pi$. For this, he used algorithms (38) and (41).

1988. Jonathan Borwein and Peter Borwein developed the series

$$
\frac{1}{\pi}=12 \sum_{n=0}^{\infty} \frac{(-1)^{n}(6 n) !}{(n !)^{3}(3 n) !} \frac{(A+B n)}{C^{n+1 / 2}}
$$

where

$$
\begin{aligned}
& A=212,175,710,912 \sqrt{61}+1,657,145,277,365, \\
& B=13,773,980,892,672 \sqrt{61}+107,578,229,802,750, \\
& C=[5,280(236,674+30,303 \sqrt{61})]^{3} .
\end{aligned}
$$

Each additional term of the series adds roughly 31 digits.

1988. Dario Castellanos gave the following approximation:

$$
\pi \simeq\left(\frac{77,729}{254}\right)^{1 / 5}=3.1415926541 \ldots
$$

May 1989. David Volfovich Chudnovsky (born 1947) and Gregory Volfovich Chudnovsky (born 1952) have published hundreds of research papers and books on number theory and mathematical physics. Gregory solved Hilbert's tenth problem at the age of 17. They on CRAY-2 and IBM 3090/VF computed 480,000,000 decimal places of $\pi$. 
June 1989. David and Gregory Chudnovsky on IBM 3090 computed 535,339,270 decimal places of $\pi$.

July 1989. Yasumasa Kanada and Yoshiaki Tamura on HITAC S-820/80 computed $536,870,898$ decimal places of $\pi$. For this, they used algorithm (38).

August 1989. David and Gregory Chudnovsky developed the following rapidly convergent generalized hypergeometric series:

$$
\frac{1}{\pi}=12 \sum_{n=0}^{\infty}(-1)^{n} \frac{(6 n) !}{(n !)^{3}(3 n) !} \frac{13,591,409+545,140,134 n}{\left(640,320^{3}\right)^{n+1 / 2}} .
$$

Each additional term of the series adds roughly 15 digits. This series is an improved version to that of Ramanujan's (35). It was used by the Chudnovsky brothers to calculate more than one billion (to be exact 1,011,196,691) digits on IBM 3090.

November 1989. Yasumasa Kanada and Yoshiaki Tamura on HITAC S-820/80 computed 1,073,740,799 decimal places of $\pi$. For this, they used algorithms (38) and (41).

August 1991. David and Gregory Chudnovsky used a home made parallel computer (they called it $m$ zero, where $m$ stands for machine, and zero for the success) to obtain $2,260,000,000$ decimal places of $\pi$. For this they used series (42).

1991. David Boll discovered an occurrence of $\pi$ in the Mandelbrot set fractal.

1991. Jonathan Borwein and Peter Borwein improved on the Salamin-Brent algorithm (38). Set $a_{0}=1 / 3$ and $s_{0}=(\sqrt{3}-1) / 2$. Iterate

$$
\begin{aligned}
& r_{k+1}=\frac{3}{1+2\left(1-s_{k}^{3}\right)^{1 / 3}}, \\
& s_{k+1}=\frac{r_{k+1}-1}{2}, \\
& a_{k+1}=r_{k+1}^{2} a_{k}-3^{k}\left(r_{k+1}^{2}-1\right) .
\end{aligned}
$$

Then $1 / a_{k}$ converges cubically to $\pi$, i.e., each iteration approximately triples the number of correct digits.

Among the several other known iterative schemes, we list the following two which are easy to implement on a computer: Set $a_{0}=1 / 2$ and $s_{0}=5(\sqrt{5}-2)$. Iterate

$$
\begin{aligned}
& x_{n+1}=5 / s_{n}-1, \\
& y_{n+1}=\left(x_{n+1}-1\right)^{2}+7, \\
& z_{n+1}=\left(\frac{1}{2} x_{n+1}\left(y_{n+1}+\sqrt{y_{n+1}^{2}-4 x_{n+1}^{3}}\right)\right)^{1 / 5}, \\
& a_{n+1}=s_{n}^{2} a_{n}-5^{n}\left(\frac{s_{n}^{2}-5}{2}+\sqrt{s_{n}\left(s_{n}^{2}-2 s_{n}+5\right)}\right), \\
& s_{n+1}=\frac{25}{\left(z_{n+1}+x_{n+1} / z_{n+1}+1\right)^{2} s_{n}} .
\end{aligned}
$$

Then $a_{k}$ converges quintically to $1 / \pi$, i.e., each iteration approximately quintuples the number of correct digits, and $0<a_{n}-1 / \pi<16 \cdot 5^{n} \cdot e^{-5^{n}} \pi$. 


$$
\text { Set } \begin{aligned}
a_{0} & =1 / 3, r_{0}=(\sqrt{3}-1) / 2 \text { and } s_{0}=\left(1-r_{0}^{3}\right)^{1 / 3} . \text { Iterate } \\
t_{n+1} & =1+2 r_{n} \\
u_{n+1} & =\left(9 r_{n}\left(1+r_{n}+r_{n}^{2}\right)\right)^{1 / 3} \\
v_{n+1} & =t_{n+1}^{2}+t_{n+1} u_{n+1}+u_{n+1}^{2}, \\
w_{n+1} & =\frac{27\left(1+s_{n}+s_{n}^{2}\right)}{v_{n+1}} \\
a_{n+1} & =w_{n+1} a_{n}+3^{2 n-1}\left(1-w_{n+1}\right), \\
s_{n+1} & =\frac{\left(1-r_{n}\right)^{3}}{\left(t_{n+1}+2 u_{n+1}\right) v_{n+1}} \\
r_{n+1} & =\left(1-s_{n+1}^{3}\right)^{1 / 3} .
\end{aligned}
$$

Then $a_{k}$ converges nonically to $1 / \pi$, i.e., each iteration approximately multiplies the number of correct digits by nine.

1993. Jonathan Borwein and Peter Borwein developed the series

$$
\frac{\sqrt{-C^{3}}}{\pi}=\sum_{m=0}^{\infty} \frac{(6 m) !}{(3 m) !(m !)^{3}} \frac{A+m B}{C^{3 m}}
$$

where

$$
\begin{aligned}
A= & 63,365,028,312,971,999,585,426,220 \\
& +28,337,702,140,800,842,046,825,600 \sqrt{5} \\
& +384 \sqrt{5}(10,891,728,551,171,178,200,467,436,212,395,209,160,385,656,017 \\
& +4,870,929,086,578,810,225,077,338,534,541,688,721,351,255,040 \sqrt{5})^{1 / 2}, \\
B= & 7,849,910,453,496,627,210,289,749,000 \\
& +3,510,586,678,260,932,028,965,606,400 \sqrt{5} \\
& +2,515,968 \sqrt{3,110}(6,260,208,323,789,001,636,993,322,654,444,020,882,161 \\
& +2,799,650,273,060,444,296,577,206,890,718,825,190,235 \sqrt{5})^{1 / 2}
\end{aligned}
$$

and

$$
\begin{aligned}
C= & -214,772,995,063,512,240-96,049,403,338,648,032 \sqrt{5} \\
& -1,296 \sqrt{5}(10,985,234,579,463,550,323,713,318,473 \\
& +4,912,746,253,692,362,754,607,395,912 \sqrt{5})^{1 / 2} .
\end{aligned}
$$

Each additional term of the series adds approximately 50 digits. However, computation of this series on a computer does not seem to be easy.

May 1994. David and Gregory Chudnovsky used a home made parallel computer $m$ zero to obtain 4,044,000,000 decimal places of $\pi$. For this they used series (42). 
June 1995. Yasumasa Kanada and Daisuke Takahashi on HITAC S-3800/480 (dual CPU) computed 3,221,225,466 decimal places of $\pi$. For this, they used algorithms (38) and (41).

August 1995. Yasumasa Kanada and Daisuke Takahashi on HITAC S-3800/480 (dual CPU) computed 4,294,967,286 decimal places of $\pi$. For this, they used algorithms (38) and (41).

October 1995. Yasumasa Kanada and Daisuke Takahashi on HITAC S-3800/480 (dual CPU) computed $6,442,450,938$ decimal places of $\pi$. For this, they used algorithms (38) and (41).

1995. David Bailey, Peter Borwein and Simon Plouffe developed the following formula (known as BBP formula) to compute the $n$th hexadecimal digit (base 16) of $\pi$ without having the previous $n-1$ digits

$$
\pi=\sum_{m=0}^{\infty} \frac{1}{16^{m}}\left(\frac{4}{8 m+1}-\frac{2}{8 m+4}-\frac{1}{8 m+5}-\frac{1}{8 m+6}\right)
$$

To show the validity of (43), for any $k<8$, we have

$$
\int_{0}^{1 / \sqrt{2}} \frac{x^{k-1}}{1-x^{8}} d x=\int_{0}^{1 / \sqrt{2}} \sum_{m=0}^{\infty} x^{k-1+8 m} d x=\frac{1}{2^{k / 2}} \sum_{m=0}^{\infty} \frac{1}{16^{m}(8 m+k)},
$$

therefore

$$
\begin{gathered}
\sum_{m=0}^{\infty} \frac{1}{16^{m}}\left(\frac{4}{8 m+1}-\frac{2}{8 m+4}-\frac{1}{8 m+5}-\frac{1}{8 m+6}\right) \\
\quad=\int_{0}^{1 / \sqrt{2}} \frac{4 \sqrt{2}-8 x^{3}-4 \sqrt{2} x^{4}-8 x^{5}}{1-x^{8}} d x .
\end{gathered}
$$

Substituting $u=\sqrt{2} x$ in equation (44), we obtain

$$
\int_{0}^{1} \frac{16 u-16}{u^{4}-2 u^{3}+4 u-4} d u=\int_{0}^{1} \frac{4 u}{u^{2}-2} d u-\int_{0}^{1} \frac{4 u-8}{u^{2}-2 u+2} d u=\pi
$$

The discovery of this formula came as a surprise. For centuries, it had been assumed that there was no way to compute the $n$th digit of $\pi$ without calculating all of the preceding $n-1$ digits. Since this discovery, many such formulas for other irrational numbers have been discovered. Such formulas have been called as spigot algorithms because, like water dripping from a spigot, they produce digits that are not reused after they are calculated.

1996. Simon Plouffe discovered an algorithm for the computation of $\pi$ in any base. Later he expressed regrets for having shared credit for his discovery of this formula with Bailey and Borwein.

March 1996. David and Gregory Chudnovsky used a home made parallel computer $m$ zero to obtain 8,000,000,000 decimal places of $\pi$. For this, they used series (42). They said 'we are looking for the appearance of some rules that will distinguish the digits of $\pi$ from other numbers, i.e., if someone were to give you a million digits from somewhere in $\pi$, could you tell it was from $\pi$ ? The digits of $\pi$ form the most nearly perfect random sequence of digits that has ever been discovered. However, each digit appears to be orderly. If a single 
digit in $\pi$ were to be changed anywhere between here and infinity, the resulting number would no longer be $\pi$, it would be garbage. Around the three-hundred-millionth decimal place of $\pi$, the digits go 88888888 -eight eights pop up in a row. Does this mean anything? It appears to be random noise. Later, ten sixes erupt: 6666666666 . What does this mean? Apparently nothing, only more noise. Somewhere past the half-million mark appears the string 123456789. It is an accident, as it were. We do not have a good, clear, crystallized idea of randomness. It cannot be that $\pi$ is truly random. Actually, a truly random sequence of numbers has not yet been discovered'

1996. Gosper posted the following fascinating formula

$$
\lim _{n \rightarrow \infty} \prod_{m=n}^{2 n} \frac{\pi}{2 \tan ^{-1} m}=4^{1 / \pi}=1.554682 \ldots .
$$

April 1997. Yasumasa Kanada and Daisuke Takahashi on HITACHI SR2201 (1,024 CPU) computed 17,179,869,142 decimal places of $\pi$. For this, they used algorithms (38) and (41).

July 1997. Yasumasa Kanada and Daisuke Takahashi on HITACHI SR2201 (1,024 CPU) computed 51,539,600,000 decimal places of $\pi$. The computation tool just over 29 hours, at an average rate of nearly 500,000 digits per second. For this, they used algorithms (38) and (41).

1997. Fabrice Bellard developed the following formula:

$$
\begin{aligned}
\pi= & \frac{1}{2^{6}} \sum_{m=0}^{\infty} \frac{(-1)^{m}}{2^{10 m}} \\
& \times\left(-\frac{2^{5}}{4 m+1}-\frac{1}{4 m+3}+\frac{2^{8}}{10 m+1}-\frac{2^{6}}{10 m+3}-\frac{2^{2}}{10 m+5}-\frac{2^{2}}{10 m+7}+\frac{1}{10 m+9}\right),
\end{aligned}
$$

which can used to compute the $n$th digit of $\pi$ in base 2 . It is about $43 \%$ faster then (43). The following exotic formula is also due to him:

$$
\pi=\frac{1}{740,025}\left[\sum_{m=1}^{\infty} \frac{3 P(m)}{\left(\begin{array}{c}
7 m \\
2 m
\end{array}\right) 2^{m-1}}-20,379,280\right],
$$

where

$$
\begin{aligned}
P(m)= & -885,673,181 m^{5}+3,125,347,237 m^{4}-2,942,969,225 m^{3} \\
& +1,031,962,795 m^{2}-196,882,274 m+10,996,648 .
\end{aligned}
$$

April 1999. Yasumasa Kanada and Daisuke Takahashi on HITACHI SR8000 (64 of 128 nodes) computed $68,719,470,000$ decimal places of $\pi$. For this, they used algorithms (38) and (41).

September 1999. Yasumasa Kanada and Daisuke Takahashi on HITACHI SR8000/MPP (128 nodes) computed 206,158,430,000, i.e., 206 billion decimal places of $\pi$. For this, they used algorithms (38) and (41). 
1999. Leo Jerome Lange developed the following continued fraction of $\pi$ :

$$
\pi=3+\frac{1^{2}}{6+\frac{3^{2}}{6+\frac{5^{2}}{6+\frac{7^{2}}{6+\cdots}}} .}
$$

2000. J. Munkhammar gave the following formula which is related to Viéte's (6):

$$
\pi=\lim _{n \rightarrow \infty} 2^{n+1} \sqrt{2-\frac{\sqrt{2+\sqrt{2+\sqrt{2+\cdots+\sqrt{2}}}}}{n}},
$$

which as a recurrence relation can be written as $\pi=\lim _{n \rightarrow \infty} 2^{n+1} a_{n}$, where $a_{0}=\sqrt{2}$, and

$$
a_{n}=\sqrt{\left(\frac{1}{2} a_{n-1}\right)^{2}+\left[1-\sqrt{1-\left(\frac{1}{2} a_{n-1}\right)^{2}}\right]^{2}} .
$$

Another closely related formula is

$$
\pi=2 \lim _{n \rightarrow \infty} \sum_{m=1}^{n} \sqrt{\left[\sqrt{1-\left(\frac{m-1}{n}\right)^{2}}-\sqrt{1-\left(\frac{m}{n}\right)^{2}}\right]^{2}+\frac{1}{n^{2}}} .
$$

2001. Robert Palais believes that the notation $\pi$ is wrongly used right from the beginning. According to him, some suitable symbol (now popular as tau $\tau$ ) must have been used for $2 \pi$. He justifies his claim by giving several formulas where $\tau$ appears naturally rather than just $\pi$. For some people, June 28, is Tau's Day and they celebrate.

November 2002. Yasumasa Kanada used Machin-like formulas (33) and (39) to compute the value of $\pi$ to 1,241,100,000,000 decimal places. The calculation took more than 600 hours on 64 nodes of a HITACHI SR8000/MPP supercomputer. The work was done at the Department of Information Science at the University of Tokyo. For this, he used arctangent formulas (33) and (39).

2004. Daniel Tammet, at age 25 , recited 22,514 decimal places of $\pi$, scoring the European record. For an audience at the Museum of the History of Science in Oxford, he said these numbers aloud for 5 hours and 9 minutes. Unfortunately, he made his first mistake at position 2,965 and did not correct this error immediately and without outside help, but only after he was told that there was a mistake.

2005. Stephen K. Lucas found that

$$
\pi=\frac{355}{113}-\int_{0}^{1} \frac{x^{8}(1-x)^{8}\left(25+816 x^{2}\right)}{3,164\left(1+x^{2}\right)} d x
$$

Several other integral formulas of this type are known, here we give the following:

$$
\pi=\frac{741,269,838,109}{235,953,517,800}-\int_{0}^{1} \frac{x^{16}(1-x)^{16}}{64\left(1+x^{2}\right)} d x
$$


which gives $3.14159265358955<\pi$. If we substitute $x=1$ in the above integral and note that

$$
\int_{0}^{1} \frac{1}{128} x^{16}(1-x)^{16} d x=\frac{1}{2,538,963,567,360}
$$

then it follows that $\pi<3.14159265358996$.

November 2005. Chao Lu, a chemistry student, at age 23, broke the Guiness record by reciting $\pi$ from memory to 67,890 places. For this, he practiced for 4 years. The attempt lasted $24 \mathrm{hrs} 4 \mathrm{~min}$ and was recorded on 26 video tapes. The attempt was witnessed by 8 officials, math professors and 20 students.

2005. Kate Bush in the song $\pi$ (in her album Aerial) sings the number to its 137 th decimal place (though she omits the 79th to 100th decimal places).

October 2006. Akira Haraguchi a retired engineer from Chiba recited $\pi$ from memory to 54,000 digits in September 2004, 68,000 digits in December 2004, 83,431 digits in July 2005, and 100,000 digits in October 2006. He accomplished the last recitation in 16 1/2hours in Tokyo. He says memorization of the digits of $\pi$ is 'the religion of the universe'.

2006. Simon Plouffe found the following curious formula:

$$
\pi=72 \sum_{k=1}^{\infty} \frac{1}{k\left(e^{k \pi}-1\right)}-96 \sum_{k=1}^{\infty} \frac{1}{k\left(e^{2 k \pi}-1\right)}+24 \sum_{k=1}^{\infty} \frac{1}{k\left(e^{4 k \pi}-1\right)} .
$$

2008. In Midnight (tenth episode of the fourth series of British science fiction television series Doctor Who), the character, the businesswoman, Sky Silvestry mimics the speech of The Doctor by repeating the square root of $\pi$ to 30 decimal places 1.77245385090551602729816748334 .

2008. Syamal K. Sen and Ravi P. Agarwal suggested four Matlab based procedures, $v i z$, (i) Exhaustive search, (ii) Principal convergents of continued fraction based procedure, (iii) Best rounding procedure for decimal (rational) approximation, and (iv) Continued fraction based algorithm with intermediate convergents. While the first procedure is exponential-time, the remaining three are polynomial-time. Roughly speaking, they have demonstrated that the absolute best $k$-digit rational approximation of $\pi$ will be as good as $2 k$-digit decimal approximation of $\pi$. The absolute best $k$-digit rational approximation is most desired for error-free computation involving $\pi$ /any other irrational number.

2008. Syamal K. Sen, Ravi P. Agarwal and Ghoolam A. Shaykhian have demonstrated through numerical experiment using Matlab that $\pi$ has always scored over $\phi$ (golden ratio), as a source of uniformly distributed random numbers, statistically in one-dimensional Monte Carlo (M.C.) integration; whether $\pi$ fares better than $\phi$ for double, triple and higher dimensional M.C. integration or not deserves exploration.

2009. Syamal K. Sen, Ravi P. Agarwal and Ghoolam A. Shaykhian compared the four procedures they proposed in (2008) for computing best $k$-digit rational approximations of irrational numbers in terms of quality (error) and cost (complexity). They have stressed on the fact that ultra-high-speed computing along with abundance of unused computing power allows employing an exponential-time algorithm for most real-world problems. This obviates the need for acquiring and employing the mathematical knowledge involving principal and intermediate convergents computed using a polynomial-time algorithm for practical problems. Since $\pi$ is the most used irrational number in the physical world, the 
simple concise Matlab program would do the job wherever $\pi$ /any other irrational number is involved.

2009. Syamal K. Sen, Ravi P. Agarwal and Raffela Pavani have provided, using Matlab, the best possible rational bounds bracketing $\pi$ /any irrational number with absolute error and the time complexity involved. Any better bounds are impossible. In these rational bounds, either the lower bound or the upper bound will always be the absolute best rational approximation. The absolute error computed provides the overall error bounds in an error-free computational environment involving $\pi$ /any other irrational number.

2009. Tue N. Vu has given Machin-type formula (http://seriesmathstudy.com/sms/ machintypetv): For each positive integer $n$,

$$
\frac{\pi}{4}=\tan ^{-1}\left(\frac{1}{4+2 n}\right)+\tan ^{-1}\left(\frac{1}{5+2 n}\right)+\sum_{k=0}^{n}\left[\tan ^{-1}\left(\frac{1}{2(2+k)^{2}}\right)+\tan ^{-1}\left(\frac{2}{(3+2 k)^{2}}\right)\right] .
$$

2009. Cetin Hakimoglu-Brown developed the following expansion:

$$
\pi=\frac{\sqrt{3}}{6^{5}} \sum_{k=0}^{\infty} \frac{((4 k) !)^{2}(6 k) !}{9^{k+1}(12 k) !(2 k) !}\left(\frac{127,169}{12 k+1}-\frac{1,070}{12 k+5}-\frac{131}{12 k+7}+\frac{2}{12 k+11}\right),
$$

which can be written as

$$
\pi=\frac{\sqrt{3}}{1,155} \sum_{k=0}^{\infty} \frac{(4 k) !\left(671,840 k^{3}+1,289,936 k^{2}+782,458 k+150,835\right)}{(72)^{4 k+1}(13 / 12)_{k}(17 / 12)_{k}(19 / 12)_{k}(23 / 12)_{k}},
$$

where $(x)_{k}=x(x+1)(x+2) \cdots(x+k-1)$ is the Pochhammer notation. He also gave the expansion

$$
\pi=\frac{1}{2^{10} \sqrt{3}} \sum_{k=0}^{\infty} \frac{1}{\left(\begin{array}{c}
8 k \\
4 k
\end{array}\right) 9^{k}}\left(\frac{5,717}{8 k+1}-\frac{413}{8 k+3}-\frac{45}{8 k+5}+\frac{5}{8 k+7}\right) .
$$

August 2009. Daisuke Takahashi et al. used a massive parallel computer called the T2K Tsukuba System to compute $\pi$ to 2,576,980,377,524 decimal places in 73 hours 36 minutes. For this, they used algorithms (38) and (41).

December 2009. Fabrice Bellard used Chudnovsky brothers series (42) to compute $2,699,999,990,000$, i.e., 2.7 trillion decimal places of $\pi$ in 131 days. For this, he used a single desktop PC, costing less than $\$ 3,000$.

August 2010. Shigeru Kondo and Alexander J. Yee used Chudnovsky brothers series (42) to compute $5,000,000,000,000$, i.e., 5 trillion decimal places of $\pi$ in 90 days. For this, they used a server-class machine running dual Intel Xeons, equipped with $96 \mathrm{~GB}$ of RAM.

2010. Michael Keith used 10,000 digits of $\pi$ to establish a new form of constrained writing, where the word lengths are required to represent the digits of $\pi$. His book contains a collection of poetry, short stories, a play, a movie script, crossword puzzles and other surprises.

2011. Syamal K. Sen and Ravi P. Agarwal in their monograph systematically organized their work of 2008 and 2009 on $\pi$ and other irrational numbers. They also included several examples to illustrate the importance of their findings. 
2011. During the auction for Nortel's portfolio of valuable technology patents, Google made a series of strange bids based on mathematical and scientific constants, including $\pi$.

October 2011. Shigeru Kondo and Alexander J. Yee used Chudnovsky brothers series (42) to compute 10,000,000,000,050, i.e., 10 trillion decimal places of $\pi$ in 371 days.

2011. Cristinel Mortici improved Gurland's bounds (36) to $\alpha_{n}<\pi<\beta_{n}, n \geq 1$ where

$$
\alpha_{n}=\left(\frac{n+\frac{1}{4}}{n^{2}+\frac{1}{2} n+\frac{3}{32}}+\frac{9}{2,048 n^{5}}-\frac{45}{8,192 n^{6}}\right)\left(\frac{(2 n) ! !}{(2 n-1) ! !}\right)^{2}
$$

and

$$
\beta_{n}=\left(\frac{n+\frac{1}{4}}{n^{2}+\frac{1}{2} n+\frac{3}{32}}+\frac{9}{2,048 n^{5}}\right)\left(\frac{(2 n) ! !}{(2 n-1) ! !}\right)^{2} .
$$

It follows that

$$
\alpha_{n}=\pi+O\left(\frac{1}{n^{6}}\right) \text { and } \beta_{n}=\pi+O\left(\frac{1}{n^{5}}\right)
$$

2012. Long Lin has improved Mortici's bounds to $\lambda_{n}<\pi<\mu_{n}, n \geq 1$ where

$$
\lambda_{n}=\left(1+\frac{1}{4 n}-\frac{3}{32 n^{2}}+\frac{3}{128 n^{3}}+\frac{3}{2,048 n^{4}}-\frac{33}{8,192 n^{5}}-\frac{39}{65,536 n^{6}}\right) \frac{2}{2 n+1}\left(\frac{(2 n) ! !}{(2 n-1) ! !}\right)^{2}
$$

and

$$
\mu_{n}=\left(1+\frac{1}{4 n}-\frac{3}{32 n^{2}}+\frac{3}{128 n^{3}}+\frac{3}{2,048 n^{4}}\right) \frac{2}{2 n+1}\left(\frac{(2 n) ! !}{(2 n-1) ! !}\right)^{2}
$$

It follows that

$$
\lambda_{n}=\pi+O\left(\frac{1}{n^{7}}\right) \text { and } \mu_{n}=\pi+O\left(\frac{1}{n^{5}}\right) .
$$

He has also obtained the higher order bounds $\delta_{n}<\pi<\omega_{n}, n \geq 1$ where

$$
\delta_{n}=\frac{1}{n} \exp \left\{-\frac{1}{4 n}+\frac{1}{96 n^{3}}-\frac{1}{320 n^{5}}+\frac{17}{7,168 n^{7}}-\frac{31}{9,216 n^{9}}\right\}\left(\frac{(2 n) ! !}{(2 n-1) ! !}\right)^{2}
$$

and

$$
\mu_{n}=\frac{1}{n} \exp \left\{-\frac{1}{4 n}+\frac{1}{96 n^{3}}-\frac{1}{320 n^{5}}+\frac{17}{7,168 n^{7}}\right\}\left(\frac{(2 n) ! !}{(2 n-1) ! !}\right)^{2} .
$$

It follows that

$$
\delta_{n}=\pi+O\left(\frac{1}{n^{11}}\right) \text { and } \mu_{n}=\pi+O\left(\frac{1}{n^{9}}\right)
$$




\section{Conclusions}

No number system can capture $\pi$ exactly. We are deeply and almost completely involved in the conventional decimal number system in representing any real quantity. This is not the only number system for the representation. There are other number systems such as binary, octal, hexadecimal, binary-coded decimal, negative radix, $p$-adic and modular number systems. If the circumference of a circle is exactly represented, then its diameter will not have exact representation and vice versa.

Reading the mathematicians in pre-computer days. An important focus of this paper is that the reader besides, however, knowing the usual chronology of the events in the life of $\pi$, could get a feel and also read how the mind of a mathematician has been working when he ponders over $\pi$ either independently without much knowledge/concern of what has been done in the past or with considerable knowledge of the work done by his predecessors. Hyper-computers ( $10^{18}$ flops) of 2012 were completely non-existence and even beyond the imagination of all the mathematicians/scientists until almost the mid-twentieth century. Also, publication machinery was too poor until the beginning of the twentieth century. Consequently, all the work on $\pi$ that has been carried out during thousands of years prior to the twentieth century was not a monotonic improvement in the $\pi$ value as well as in the exploration of its wonderful character. Many have worked on $\pi$ standalone while others have contributed with some prior knowledge of the earlier work. All of them were severely handicapped due to the non-existence of today's ultra-high speed computers. They entirely depended on their ingenuity and on whatever negligible computing device they had. It is really interesting under this environment to read these scientists/mathematicians and realize how fortunate we are in the gigantic computer age. All that has been done during the last 20 years (1990-2010) amounts to much more than what has been achieved during the past several millennia.

Matlab is well-suited to check/evaluate merits of all past $\pi$ formulas. Widely used userfriendly Matlab that needs no formal programming knowledge along with the vpa (variable precision arithmetic) and format long $g$ commands can be used to easily and readily check all that has been done during the past several thousand years and possibly appreciate the inherent intellectual import of the bygone scientists (having practically no computing device) and their expected pitfalls, bias and incorrect beliefs.

Checking exactness of billions of digits of $\pi$ is difficult. Are all the billions of digit of $\pi$ computed $100 \%$ error-free? We are familiar with the age-old proverb that 'To err is human (living being)'. Maybe a new proverb 'Not to err is computer (non-living being)' can be taken as true in the modern computer age. Here, 'err' means mistake. The arithmetic operations, particularly subtraction operations of two nearly equal numbers, involved in a formula could be sometimes error introducer. However, different computers with different formulas used to compute $\pi$ would help verification and obviate possible error in computation.

Computing nth decimal digit exactly always without preceding digits seems yet an open computational problem. While probabilistically one may determine the $n$th digit of $\pi$ without computing the preceding $n-1$ digits, obtaining $n$th digit exactly (correctly) always for any $n$ does not seem to be possible without a large precision. It seems yet an open computational problem that needs exploration. Thus, formulas such as (43) seem more of theoretical/academic interest than of practical usage as of now. 
PI for testing performance and stability of a computer. Super PI is a computer program that calculates $\pi$ to a specified number of digits after decimal point up to a maximum of 32 million digits. It uses the Gauss-Legendre algorithm and is a Windows port of the program used by Yasumasa Kanada in 1995 to compute $\pi$ to $2^{32}$ digits. Super PI is used by many overclockers to test the performance and stability of their computers. Overclocking is the process of making a computer run faster than the clock frequency specified by the manufacturer by modifying system parameters.

\section{Competing interests}

The authors declare that they have no competing interests.

\section{Authors' contributions}

All authors contributed equally and read and approved the final manuscript.

\section{Author details}

'Department of Mathematics, Texas A\&M University-Kingsville, Kingsville, TX, 78363, USA. ${ }^{2} 1540$ Ravena Street, Bethlehem, PA 18015, USA. ${ }^{3}$ GVP-Prof. V. Lakshmikantham Institute for Advanced Studies, \#1-83-21/3, Sector 8, MVP Colony, Visakhapatnam, A.P., India.

\section{Acknowledgements}

Dedicated to V Lakshmikantham (1924-2012).

Received: 22 January 2013 Accepted: 25 March 2013 Published: 11 April 2013

\section{References}

1. Adamchik, V, Wagon, S: A simple formula for $\pi$. Am. Math. Mon. 104, 852-855 (1997)

2. Adamchik, V, Wagon, S: Pi: a 2000-year search changes direction. Educ. Res. 5, 11-19 (1996)

3. Ahmad, A: On the $\pi$ of Aryabhata I. Ganita Bharati 3, 83-85 (1981)

4. Akira, $H$ : History of $\pi$. Kyoiku Tosho, Osaka (1980)

5. Al-Kashi, J: Treatise on the Circumference of the Circle (1424)

6. Almkvist, G: Many correct digits of $\pi$, revisited. Am. Math. Mon. 104, 351-353 (1997)

7. Anderson, DV: A polynomial for $\pi$. Math. Gaz. 55, 67-68 (1971)

8. Anonymous: Cyclometry and Circle-Squaring in a Nutshell. Simpkin, Marshall \& Co., Stationer's Hall Court, London (1871)

9. Arndt, J: Cryptic Pi related formulas. http://www.jjj.de/hfloat/pise.dvi

10. Arndt, J, Haenel, C: $\pi$-Unleashed. Springer, Berlin (2000)

11. Assmus, EF: Pi. Am. Math. Mon. 92, 213-214 (1985)

12. Backhouse, N: Note 79.36. Pancake functions and approximations to $\pi$. Math. Gaz. 79, 371-374 (1995)

13. Badger, L: Lazzarini's lucky approximation of $\pi$. Math. Mag. 67, 83-91 (1994)

14. Bai, S: An exploration of Liu Xin's value of $\pi$ from Wang Mang's measuring vessel. Sugaku-shi Kenkyu 116, 24-31 (1988)

15. Bailey, DH: Numerical results on the transcendence of constants involving $\pi, e$, and Euler's constant. Math. Comput. 50, 275-281 (1988)

16. Bailey, $\mathrm{DH}$ : The computation of $\pi$ to $29,360,000$ decimal digits using Borweins' quartically convergent algorithm. Math. Comput. 50, 283-296 (1988)

17. Bailey, DH, Borwein, JM, Borwein, PB, Plouffe, S: The quest for pi. Math. Intell. 19, 50-57 (1997)

18. Bailey, DH, Borwein, PB, Plouffe, S: On the rapid computation of various polylogarithmic constants. Math. Comput. 66, 903-913 (1997)

19. Beck, G, Trott, M: Calculating Pi from antiquity to modern times. http://library.wolfram.com/infocenter/Demos/107/

20. Beckmann, P: A History of $\pi$. St Martin's, New York (1971)

21. Bellard, F: Fabrice Bellard's, Pi page. http://bellard.org/pi/

22. Berggren, L, Borwein, JM, Borwein, PB: Pi: A Source Book, 3rd edn. Springer, New York (2004)

23. Beukers, F: A rational approximation to $\pi$. Nieuw Arch. Wiskd. 5, 372-379 (2000)

24. Blatner, D: The Joy of П. Penguin, Toronto (1997)

25. Bokhari, N: Piece of Pi. Dandy Lion, San Luis Obispo (2001)

26. Boll, D: Pi and the Mandelbrot set. http://www.pi314.net/eng/mandelbrot.php

27. Borwein, JM, Bailey, DH, Girgensohn, R: Experimentation in Mathematics: Computational Paths to Discovery AK Peters, Wellesley (2004)

28. Borwein, JM, Borwein, PB: A very rapidly convergent product expansion for $\pi$. BIT Numer. Math. 23, $538-540$ (1983)

29. Borwein, JM, Borwein, PB: Cubic and higher order algorithms for $\pi$. Can. Math. Bull. 27, 436-443 (1984)

30. Borwein, JM, Borwein, PB: Explicit algebraic nth order approximations to $\pi$. In: Singh, SP, Burry, JHW, Watson, B (eds.) Approximation Theory and Spline Functions, pp. 247-256. Reidel, Dordrecht (1984)

31. Borwein, JM, Borwein, PB: The arithmetic-geometric mean and fast computation of elementary functions. SIAM Rev. 26, 351-365 (1984)

32. Borwein, JM, Borwein, PB: An explicit cubic iteration for $\pi$. BIT Numer. Math. 26, 123-126 (1986)

33. Borwein, JM, Borwein, PB: More quadratically converging algorithms for $\pi$. Math. Comput. 46, $247-253$ (1986)

34. Borwein, JM, Borwein, PB: Pi and the AGM - A Study in Analytic Number Theory and Computational Complexity. Wiley-Interscience, New York (1987) 
35. Borwein, JM, Borwein, PB: Explicit Ramanujan-type approximations to $\pi$ of high order. Proc. Indian Acad. Sci. Math. Sci. 97, 53-59 (1987)

36. Borwein, JM, Borwein, PB: Ramanujan's rational and algebraic series for $1 / \pi$. J. Indian Math. Soc. 51, 147-160 (1987)

37. Borwein, JM, Borwein, PB: Ramanujan and $\pi$. Sci. Am. 258, 112-117 (1988)

38. Borwein, JM, Borwein, PB: More Ramanujan-type series for 1/ $\pi$. In: Ramanujan Revisited, pp. 359-374. Academic Press, Boston (1988)

39. Borwein, JM, Borwein, PB: Approximating $\pi$ with Ramanujan's modular equations. Rocky Mt. J. Math. 19, 93-102 (1989)

40. Borwein, JM, Borwein, PB: Class number three Ramanujan type series for $1 / \pi$. J. Comput. Appl. Math. 46, 281-290 (1993)

41. Borwein, JM, Borwein, PB, Bailey, DH: Ramanujan, modular equations, and approximations to $\pi$, or how to compute one billion digits of $\pi$. Am. Math. Mon. 96, 201-219 (1989)

42. Borwein, JM, Borwein, PB, Dilcher, K: Pi, Euler numbers, and asymptotic expansions. Am. Math. Mon. 96, 681-687 (1989)

43. Borwein, JM, Borwein, PB, Garvan, F: Hypergeometric analogues of the arithmetic-geometric mean iteration. Constr. Approx. 9, 509-523 (1993)

44. Borwein, PM: The amazing number Il. Nieuw Arch. Wiskd. 1, 254-258 (2000)

45. Brent, RP: The complexity of multiple-precision arithmetic. In: Andressen, RS, Brent, RP (eds.) Complexity of Computational Problem Solving. University of Queensland Press, Brisbane (1976)

46. Brent, RP: Fast multiple-precision evaluation of elementary functions. J. ACM 23, 242-251 (1976)

47. Breuer, S, Zwas, G: Mathematical-educational aspects of the computation of $\pi$. Int. J. Math. Educ. Sci. Technol. 15, 231-244 (1984)

48. Brown, $\mathrm{CH}$ : An algorithm for the derivation of rapidly converging infinite series for universal mathematical constants. Preprint (2009)

49. Bruins, EM: With roots towards Aryabhata's $\pi$-value. Ganita Bharati 5, 1-7 (1983)

50. Carlson, BC: Algorithms involving arithmetic and geometric means. Am. Math. Mon. 78, 496-505 (1971)

51. Castellanos, D: The ubiquitous pi, part I. Math. Mag. 61, 67-98 (1988)

52. Castellanos, D: The ubiquitous pi, part II. Math. Mag. 61, 148-163 (1988)

53. Chan, J: As easy as Pi. Math Horizons, Winter 1993, 18-19

54. Choong, KY, Daykin, DE, Rathbone, CR: Rational approximations to $\pi$. Math. Comput. 25, 387-392 (1971)

55. Choong, KY, Daykin, DE, Rathbone, CR: Regular continued fractions for $\pi$ and $\gamma$. Math. Comput. 25, 403 (1971)

56. Chudnovsky, DV, Chudnovsky, GV: Approximations and complex multiplication according to Ramanujan. In: Ramanujan Revisited, pp. 375-396 \& 468-472. Academic Press, Boston, (1988)

57. Chudnovsky, DV, Chudnovsky, GV: The computation of classical constants. Proc. Natl. Acad. Sci. USA 86, 8178-8182 (1989)

58. Cohen, GL, Shannon, AG: John Ward's method for the calculation of $\pi$. Hist. Math. 8, 133-144 (1981)

59. Colzani, L: La quadratura del cerchio e dell'iperbole (The squaring of the circle and hyperbola). Universitá degli studi di Milano-Bicocca, Matematica, Milano, Italy

60. Cox, DA: The arithmetic-geometric mean of Gauss. Enseign. Math. 30, 275-330 (1984)

61. Dahse, Z: Der Kreis-Umfang für den Durchmesser 1 auf 200 Decimalstellen berechnet. J. Reine Angew. Math. 27, 198 (1944)

62. Dalzell, DP: On 22/7. J. Lond. Math. Soc. 19, 133-134 (1944)

63. Dalzell, DP: On 22/7 and 355/113. Eureka Archimed. J. 34, 10-13 (1971)

64. Datta, B: Hindu values of $\pi$. J. Asiat. Soc. Bengal 22, 25-42 (1926)

65. Davis, PJ: The Lore of Large Numbers. New Mathematical Library, vol. 6. Math. Assoc. of America, Washington (1961)

66. Delahaye, JP: Le Fascinant Nombre $\pi$. Bibliothéque Pour la Science, Belin (1997)

67. Dixon, R: The story of pi $(\pi)$. In: Mathographics. Dover, New York (1991)

68. Engels, H: Quadrature of the circle in ancient Egypt. Hist. Math. 4, 137-140 (1977)

69. Eymard, P, Lafon, JP: The Number Pi. Am. Math. Soc., Providence (1999) (Translated by S.S. Wilson)

70. Ferguson, DF: Evaluation of $\pi$. Are Shanks' figures correct? Math. Gaz. 30, $89-90$ (1946)

71. Ferguson, DF: Value of $\pi$. Nature 17, 342 (1946)

72. Finch, SR: Mathematical Constants. Cambridge University Press, Cambridge (2003)

73. Frisby, E: On the calculation of pi. Messenger Math. 2, 114 (1872)

74. Fox, L, Hayes, L: A further helping of $\pi$. Math. Gaz. 59, $38-40$ (1975)

75. Fuller, R: Circle and Square. Springfield Printing and Binding Co., Springfield (1908)

76. Genuys, F: Dix milles décimales de $\pi$. Chiffres $1,17-22$ (1958)

77. Goggins, JR: Formula for $\pi / 4$. Math. Gaz. 57, 134 (1973)

78. Goldsmith, C: Calculation of In 2 and $\pi$. Math. Gaz. 55, 434-436 (1971)

79. Goodrich, LC: Measurements of the circle in ancient China. Isis 39, $64-65$ (1948)

80. Gosper, RW: Acceleration of series. Memo no. 304., M.I.T., Artificial Intelligence Laboratory, Cambridge, Mass. (1974)

81. Gosper, RW: math-fun@cs.arizona.edu posting, Sept. (1996)

82. Gosper, RW: A product, math-fun@cs.arizona.edu posting, Sept. 27 (1996)

83. Gould, SC: What is the value of Pi. Notes and Queries, Manchester, N.H. (1888)

84. Gourdon, X, Sebah, P: Collection of series for $\pi$. http://numbers.computation.free.fr/Constants/Pi/piSeries.html

85. Greenblatt, MH: The 'legal' value of $\pi$ and some related mathematical anomalies. Am. Sci. 53, 427A-432A (1965)

86. Gregory, RT, Krishnamurthy, EV: Methods and Applications of Error-Free Computation. Springer, New York (1984)

87. Gridgeman, NT: Geometric probability and the number $\pi$. Scr. Math. 25, 183-195 (1960)

88. Guilloud, J, Bouyer, M: Un Million de Décimales de $\pi$. Commissariat á l'Energie Atomique, Paris (1974)

89. Gupta, RC: Aryabhata I's value of $\pi$. Math. Educ. 7, 17-20 (1973)

90. Gupta, RC: Madhava's and other medieval Indian values of $\pi$. Math. Educ. 9, 45-48 (1975)

91. Gupta, RC: Some ancient values of pi and their use in India. Math. Educ. 9, 1-5 (1975)

92. Gupta, RC: Lindemann's discovery of the transcendence of $\pi$ : a centenary tribute. Ganita Bharati 4, 102-108 (1982) 
93. Gupta, RC: New Indian values of $\pi$ from the 'Manava'sulba sutra'. Centaurus 31, 114-125 (1988)

94. Gupta, RC: On the values of $\pi$ from the bible. Ganita Bharati 10, 51-58 (1988)

95. Gupta, RC: The value of $\pi$ in the 'Mahabharata'. Ganita Bharati 12, $45-47$ (1990)

96. Gurland, J: On Wallis' formula. Am. Math. Mon. 63, 643-645 (1956)

97. Hall, A: On an experimental determination of pi. Messenger Math. 2, 113-114 (1873)

98. Hata, M: Improvement in the irrationality measures of $\pi$ and $\pi^{2}$. Proc. Jpn. Acad., Ser. A, Math. Sci. 68, 283-286 (1992)

99. Hata, M: Rational approximations to $\pi$ and some other numbers. Acta Arith. 63, 335-349 (1993)

100. Hayashi, T: The value of $\pi$ used by the Japanese mathematicians of the 17 th and 18 th centuries. In: Bibliotheca Mathematics, vol. 3, pp. 273-275 (1902)

101. Hayashi, T, Kusuba, T, Yano, M: Indian values for $\pi$ derived from Aryabhata's value. Hist. Sci. 37, 1-16 (1989)

102. Hermann, E: Quadrature of the circle in ancient Egypt. Hist. Math. 4, 137-140 (1977)

103. Hobson, EW: Squaring the Circle: A History of the Problem. Cambridge University Press, Cambridge (1913)

104. Huygens, C: De circuli magnitudine inventa. Christiani Hugenii Opera Varia, vol. I, pp. 384-388. Leiden (1724)

105. Huylebrouck, D: Van Ceulen's tombstone. Math. Intell. 4, 60-61 (1995)

106. Hwang, CL: More Machin-type identities. Math. Gaz. 81, 120-121 (1997)

107. Jami, C: Une histoire chinoise du nombre $\pi$. Arch. Hist. Exact Sci. 38, 39-50 (1988)

108. Jha, P: Aryabhata I and the value of $\pi$. Math. Educ. 16, 54-59 (1982)

109. Jha, SK, Jha, M: A study of the value of $\pi$ known to ancient Hindu and Jaina mathematicians. J. Bihar Math. Soc. 13 38-44 (1990)

110. Jones, W: Synopsis palmiorum matheseos, London, 263 (1706)

111. Jörg, A, Haenel, C: Pi Unleashed, 2nd edn. Springer, Berlin (2000) (Translated by C. Lischka and D. Lischka)

112. Kanada, Y: Vectorization of multiple-precision arithmetic program and $201,326,000$ decimal digits of $\pi$ calculation. In: Supercomputing: Science and Applications, vol. 2, pp. 117-128 (1988)

113. Kanada, Y, Tamura, Y, Yoshino, S, Ushiro, Y: Calculation of $\pi$ to $10,013,395$ decimal places based on the Gauss-Legendre algorithm and Gauss arctangent relation. Technical report 84-01, Computer Center, University of Tokyo (1983)

114. Keith, M: Not a Wake: A Dream Embodying (pi)'s Digits Fully for 10,000 Decimals. Vinculum Press, Baton Rouge (2010) (Diana Keith (Illustrator))

115. Knopp, K: Theory and Application of Infinite Series. Blackie, London (1951)

116. Kochansky, AA: Observationes Cyclometricae ad facilitandam Praxin accomodatae. Acta Erud. 4, 394-398 (1685)

117. Krishnamurhty, EV: Complementary two-way algorithms for negative radix conversions. IEEE Trans. Comput. 20, 543-550 (1971)

118. Kulkarni, RP: The value of $\pi$ known to Sulbasutrakaras. Indian J. Hist. Sci. 13, 32-41 (1978)

119. Laczkovich, M: On Lambert's proof of the irrationality of $\pi$. Am. Math. Mon. 104, 439-443 (1997)

120. de Lagny, F: Mémoire sur la quadrature du cercle et sur la mesure de tout arc, tout secteur et tout segment donné. In: Histoire de L'Académie Royale des Sciences. Académie des sciences, Paris (1719)

121. Lakshmikantham, V, Leela, S, Vasundhara Devi, J: The Origin and History of Mathematics. Cambridge Scientific Publishers, Cambridge (2005)

122. Lambert, JH: Mémoire sur quelques propriétés remarquables des quantités transcendantes circulaires et logarithmiques. In: Mémoires de l'Académie des Sciences de Berlin, vol. 17, pp. 265-322 (1761)

123. Lange, LJ: An elegant continued fraction for $\pi$. Am. Math. Mon. 106, 456-458 (1999)

124. Lay-Yong, L, Tian-Se, A: Circle measurements in ancient China. Hist. Math. 13, 325-340 (1986)

125. Lazzarini, M: Un' applicazione del calcolo della probabilitá alla ricerca sperimentale di un valore approssimato di $\pi$. Period. Mat. 4, 140-143 (1901)

126. Legendre, AM: Eléments de Géométrie. Didot, Paris (1794)

127. Lehmer, DH: On arctangent relations for $\pi$. Am. Math. Mon. 45, 657-664 (1938)

128. Lin, L: Further refinements of Gurland's formula for $\pi$. J. Inequal. Appl. 2013, 48 (2013). doi:10.1186/1029-242X-2013-48

129. Lindemann, F: Über die Zahl $\pi$. Math. Ann. 20, 213-225 (1882)

130. Le Lionnais, F: Les Nombres Remarquables. Hermann, Paris (1983)

131. Lucas, SK: Integral proofs that 355/113 > $\pi$. Aust. Math. Soc. Gaz. 32, 263-266 (2005)

132. Mao, Y: A short history of $\pi$ in China. Kexue 3, 411-423 (1917)

133. Maor, E: The history of $\pi$ on the pocket calculator. J. Coll. Sci. Teach. Nov., 97-99 (1976)

134. Matar, KM, Rajagopal, C: On the Hindu quadrature of the circle. J. Bombay Branch R. Asiat. Soc. $20,77-82$ (1944)

135. Mikami, Y: The Development of Mathematics in China and Japan. Chelsea, New York (1913)

136. Miel, G: An algorithm for the calculation of $\pi$. Am. Math. Mon. 86, 694-697 (1979)

137. Miel, G: Of calculations past and present: the Archimedean algorithm. Am. Math. Mon. 90, 17-35 (1983)

138. Moakes, AJ: The calculation of $\pi$. Math. Gaz. 54, 261-264 (1970)

139. Moakes, AJ: A further note on machine computation for $\pi$. Math. Gaz. 55, 306-310 (1971)

140. Mortici, C: Refinement of Gurland's formula for pi. Comput. Math. Appl. 62, 2616-2620 (2011)

141. Myers, WA: The Quadrature of the Circle, the Square Root of Two, and the Right-Angled Triangle. Wilstach, Baldwin \& Co. Printers, Cincinnati (1873)

142. Nagell, T: Irrationality of the numbers $e$ and $\pi$. In: Introduction to Number Theory, pp. 38-40. Wiley, New York (1951)

143. Nakamura, $\mathrm{K}$ : On the sprout and setback of the concept of mathematical 'proof' in the Edo period in Japan: regarding the method of calculating number $\pi$. Hist. Sci. 3, 185-199 (1994)

144. Nanjundiah, TS: On Huygens' approximation to $\pi$. Math. Mag. 44, 221-223 (1971)

145. Newman, M, Shanks, D: On a sequence arising in series for $\pi$. Math. Comput. 42, 199-217 (1984)

146. Nicholson, SC, Jeenel, J: Some comments on a NORC computation of $\pi$. Math. Tables Other Aids Comput. 9 , 162-164 (1955)

147. Niven, IM: A simple proof that $\pi$ is irrational. Bull. Am. Math. Soc. 53, 507 (1947)

148. Niven, IM: Irrational Numbers. Wiley, New York (1956) 
149. Palais, R: pi is wrong. Math. Intell. 23, 7-8 (2001)

150. Parker, JA: The Quadrature of the Circle: Setting Forth the Secrete Teaching of the Bible. Kessinger Publ., Whitefish (2010)

151. Pereira da Silva, C: A brief history of the number $\pi$. Bol. Soc. Parana. Mat. 7, 1-8 (1986)

152. Plouffe, S: Identities inspired from Ramanujan notebooks (Part 2). Apr. 2006. http://www.lacim.uqam.ca/ plouffe/inspired2.pdf

153. Posamentier, AS, Lehmann, I: Pi: A Biography of the World's Most Mysterious Number. Prometheus Books, New York (2004)

154. Preston, R: The mountains of $\pi$. The New Yorker, March 2, 36-67 (1992)

155. Puritz, CW: An elementary method of calculating $\pi$. Math. Gaz. 58, 102-108 (1974)

156. Qian, B: A study of $\pi$ found in Chinese mathematical books. Kexue 8, 114-129 and 254-265 (1923)

157. Rabinowitz, S, Wagon, S: A spigot algorithm for the digits of $\pi$. Am. Math. Mon. 102, 195-203 (1995)

158. Rajagopal, CT, Vedamurti Aiyar, TV: A Hindu approximation to $\pi$. Scr. Math. 18, 25-30 (1952)

159. Ramanujan, S: Modular equations and approximations to $\pi$. Q. J. Pure Appl. Math. 45(1914), 350-372 (1913-1914)

160. Reitwiesner, G: An ENIAC determination of $\pi$ and $e$ to more than 2,000 decimal places. Math. Tables Other Aids Comput. 4, 11-15 (1950)

161. Roy, R: The discovery of the series formula for $\pi$ by Leibniz, Gregory, and Nilakantha. Math. Mag. 63, 291-306 (1990)

162. Rutherford, W: Computation of the ratio of the diameter of a circle to its circumference to 208 places of figures. Philos. Trans. R. Soc. Lond. 131, 281-283 (1841)

163. Sagan, C: Contact. Simon \& Schuster, New York (1985)

164. Salamin, E: Computation of $\pi$ using arithmetic-geometric mean. Math. Comput. 30, 565-570 (1976)

165. Salikhov, $\vee$ : On the irrationality measure of $\pi$. Russ. Math. Surv. 53, 570-572 (2008)

166. Schepler, HC: The chronology of PI. Math. Magazine, January-February 1950: 165-170; March-April 1950: 216-228; May-June 1950: 279-283

167. Schröder, EM: Zur irrationalität von $\pi^{2}$ und $\pi$. Mitt. Math. Ges. Hamb. 13, 249 (1993)

168. Schubert, H: Squaring of the circle. Smithsonian Institution Annual Report (1890)

169. Sen, SK, Agarwal, RP: Best k-digit rational approximation of irrational numbers: pre-computer versus computer era. Appl. Math. Comput. 199, 770-786 (2008)

170. Sen, SK, Agarwal, RP: $\pi, e, \phi$ with MATLAB: Random and Rational Sequences with Scope in Supercomputing Era Cambridge Scientific Publishers, Cambridge (2011)

171. Sen, SK, Agarwal, RP, Shaykhianb, GA: Golden ratio versus pi as random sequence sources for Monte Carlo integration. Math. Comput. Model. 48, 161-178 (2008)

172. Sen, SK, Agarwal, RP, Shaykhian, GA: Best k-digit rational approximations-true versus convergent, decimal-based ones: quality, cost, scope. Adv. Stud. Contemp. Math. 19, 59-96 (2009)

173. Sen, SK, Agarwal, RP, Pavani, R: Best k-digit rational bounds for irrational numbers: pre- and super-computer era. Math. Comput. Model. 49, 1465-1482 (2009)

174. Shanks, D: Dihedral quartic approximations and series for $\pi$. J. Number Theory 14, 397-423 (1982)

175. Shanks, D, Wrench, JW Jr.: Calculation of $\pi$ to 100,000 decimals. Math. Comput. 16, 76-99 (1962)

176. Shanks, W: Contributions to Mathematics Comprising Chiefly the Rectification of the Circle to 607 Places of Decimals. Bell, London (1853)

177. Shanks, W: On the extension of the numerical value of $\pi$. Proc. R. Soc. Lond. 21, 315-319 (1873)

178. Singmaster, D: The legal values of $\pi$. Math. Intell. 7, 69-72 (1985)

179. Smith, DE: History and transcendence of pi. In: Young, WJA (ed.) Monograms on Modern Mathematics. Longmans, Green, New York (1911)

180. Smith, DE: The history and transcendence of $\pi$. In: Young, JWA (ed.) Monographs on Topics of Modern Mathematics Relevant to the Elementary Field, chapter 9, pp. 388-416. Dover, New York (1955)

181. Smith, DE, Mikami, Y: A History of Japanese Mathematics. Open-Court, Chicago (1914)

182. van Roijen Snell, W: Cyclometricus. Leiden (1621)

183. Sondow, J: A faster product for $\pi$ and a new integral for $\ln (\pi / 2)$. Am. Math. Mon. 112, $729-734$ (2005)

184. Stern, MD: A remarkable approximation to $\pi$. Math. Gaz. 69, 218-219 (1985)

185. Stevens, J: Zur irrationalität von $\pi$. Mitt. Math. Ges. Hamb. 18, 151-158 (1999)

186. Störmer, C: Sur l'application de la théorie des nombres entiers complexes á la solution en nombres rationnels $x_{1}, x_{2}, \ldots, x_{n}, c_{1}, c_{2}, \ldots, c_{n}, k$ de l'équation $c_{1} \arctan x_{1}+c_{2} \arctan x_{2}+\cdots+c_{n} \arctan x_{n}=k p / 4$. Arch. Math. Naturvidensk. 19, 75-85 (1896)

187. Takahasi, D, Kanada, Y: Calculation of $\pi$ to 51.5 billion decimal digits on distributed memory and parallel processors Trans. Inf. Process. Soc. Jpn. 39(7) (1998)

188. Tamura, Y, Kanada, Y: Calculation of $\pi$ to 4,194,293 decimals based on Gauss-Legendre algorithm. Technical report 83-01, Computer Center, University of Tokyo (1982)

189. Todd, J: A problem on arctangent relations. Am. Math. Mon. 56, 517-528 (1949)

190. Trier, PE: Pi revisited. Bull. - Inst. Math. Appl. 25, 74-77 (1989)

191. Tweddle, I: John Machin and Robert Simson on inverse-tangent series for $\pi$. Arch. Hist. Exact Sci. 42, 1-14 (1991)

192. Uhler, HS: Recalculation and extension of the modulus and of the logarithms of 2, 3, 5, 7 and 17. Proc. Natl. Acad. Sci. USA 26, 205-212 (1940)

193. Vega, G: Thesaurus Logarithmorum Completus. Leipzig (1794)

194. Viéta, F: Uriorum de rebus mathematicis responsorum. Liber VII (1593)

195. Volkov, A: Calculations of $\pi$ in ancient China: from Liu Hui to Zu Chongzhi. Hist. Sci. 4, 139-157 (1994)

196. Volkov, A: Supplementary data on the values of $\pi$ in the history of Chinese mathematics. Philos. Hist. Sci. Taiwan. J. 3, 95-120 (1994)

197. Volkov, A: Zhao Youqin and his calculation of $\pi$. Hist. Math. 24, 301-331 (1997)

198. Wagon, S: Is $\pi$ normal. Math. Intell. 7, 65-67 (1985)

199. Wells, D: The Penguin Dictionary of Curious and Interesting Numbers. Penguin, Middlesex (1986)

200. Wrench, JW Jr.: The evolution of extended decimal approximations to $\pi$. Math. Teach. 53, 644-650 (1960) 
201. Wrench, JW Jr., Smith, LB: Values of the terms of the Gregory series for arccot 5 and arccot 239 to 1,150 and 1,120 decimal places, respectively. Math. Tables Other Aids Comput. 4, 160-161 (1950)

202. Yeo, A: The Pleasures of $\pi, e$ and Other Interesting Numbers. World Scientific, Singapore (2006)

203. Zebrowski, E: A History of the Circle: Mathematical Reasoning and the Physical Universe. Rutgers University Press, Pisacataway (1999)

204. Zha, Y-L: Research on Tsu Ch'ung-Chih's approximate method for $\pi$. In: Science and Technology in Chinese Civilization, pp. 77-85. World Scientific, Teaneck (1987)

205. http://mathworld.wolfram.com/PiFormulas.html

206. http://en.wikipedia.org/wiki/Pi

207. http://en.wikipedia.org/wiki/Pi_approximations

208. en.wikipedia.org/wiki/Negative_base

209. en.wikipedia.org/wiki/Super_Pi

210. en.wikipedia.org/wiki/Overclocking

doi:10.1186/1687-1847-2013-100

Cite this article as: Agarwal et al.: Birth, growth and computation of pi to ten trillion digits. Advances in Difference Equations 2013 2013:100.

Submit your manuscript to a SpringerOpen ${ }^{\circ}$ journal and benefit from:

- Convenient online submission

- Rigorous peer review

- Immediate publication on acceptance

- Open access: articles freely available online

- High visibility within the field

- Retaining the copyright to your article 